\title{
Dissociation: a defensive maneuver?
}

Citation for published version (APA):

Giesbrecht, T. M. (2006). Dissociation: a defensive maneuver? [Doctoral Thesis, Maastricht University]. Datawyse / Universitaire Pers Maastricht. https://doi.org/10.26481/dis.20060101tg

Document status and date:

Published: 01/01/2006

DOI:

10.26481/dis.20060101tg

Document Version:

Publisher's PDF, also known as Version of record

\section{Please check the document version of this publication:}

- A submitted manuscript is the version of the article upon submission and before peer-review. There can be important differences between the submitted version and the official published version of record.

People interested in the research are advised to contact the author for the final version of the publication, or visit the DOI to the publisher's website.

- The final author version and the galley proof are versions of the publication after peer review.

- The final published version features the final layout of the paper including the volume, issue and page numbers.

Link to publication

\footnotetext{
General rights rights.

- You may freely distribute the URL identifying the publication in the public portal. please follow below link for the End User Agreement:

www.umlib.nl/taverne-license

Take down policy

If you believe that this document breaches copyright please contact us at:

repository@maastrichtuniversity.nl

providing details and we will investigate your claim.
}

Copyright and moral rights for the publications made accessible in the public portal are retained by the authors and/or other copyright owners and it is a condition of accessing publications that users recognise and abide by the legal requirements associated with these

- Users may download and print one copy of any publication from the public portal for the purpose of private study or research.

- You may not further distribute the material or use it for any profit-making activity or commercial gain

If the publication is distributed under the terms of Article $25 \mathrm{fa}$ of the Dutch Copyright Act, indicated by the "Taverne" license above, 


\section{Dissociation: A defensive maneuver?}


Promotor:

Prof. dr. H.L.G.J. Merckelbach

Beoordelingscommissie :

Prof. dr. A.T.M. Jansen (voorzitter)

Dr. D.P. Bernstein

Prof. dr. M.A. van den Hout (Universiteit Utrecht)

Prof. dr. P. Muris (Erasmus Universiteit Rotterdam)

Prof. dr. J.J. van Os

The studies presented in this dissertation were funded by the Dutch Organization for Scientific Research (NOW, The Hague, grant number 402-01-088-D). 


\section{Contents}

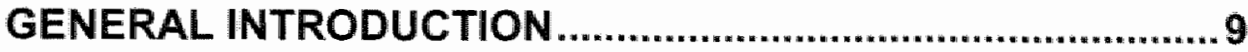

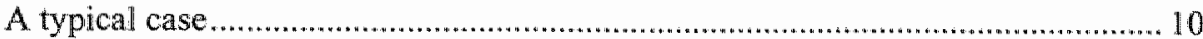

Outline of the present thesis.................................................................................... 11

Chapter 1: Dissociation and trauma: A review.................................................... 12

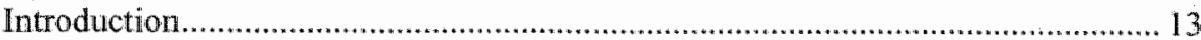

The Dissociative Experiences Scale as a measure of dissociative experiences........ 13

The causal relation between dissociation and traumatic experiences ..................... 14

Dissociative experiences and other personality traits ............................................ 18

Heritability of dissociation, cognitive failures, and fantasy proneness ................... 20

The defensive properties of dissociation.............................................................. 21

Dissociation and sleep........................................................................................ 23

Sleep experiences as an altermative explanation for the development of dissociative

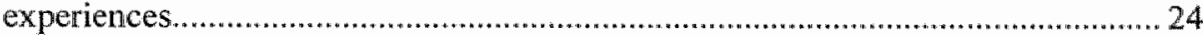

\section{PART I: DISSOCIATION AND EMOTION}

Chapter 2: Dissociation, memory commission errors, and heightened autonomic reactivity

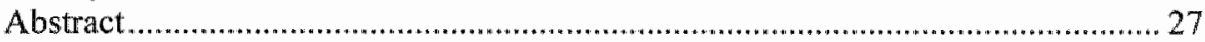

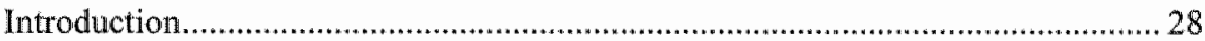

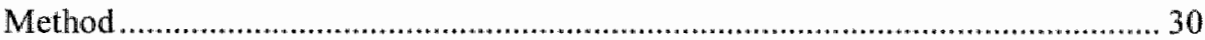

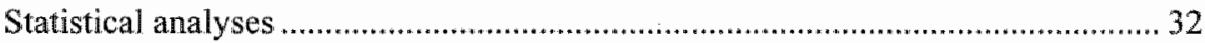

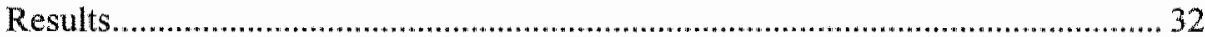

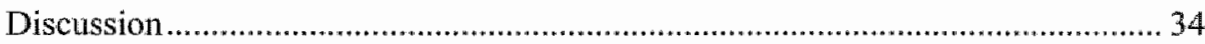

Chapter 3: Trait dissociation and stress induced cortisol responses........................ 39

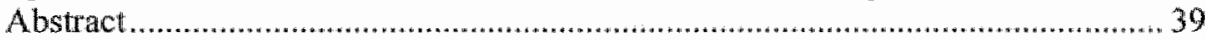

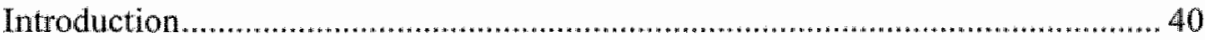

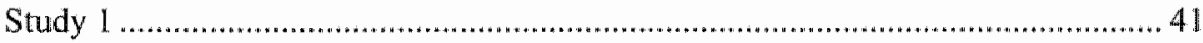

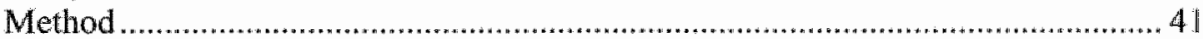

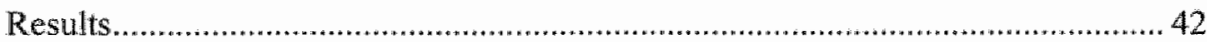

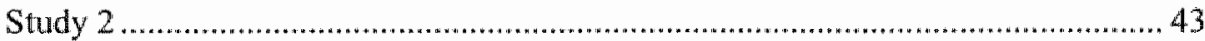

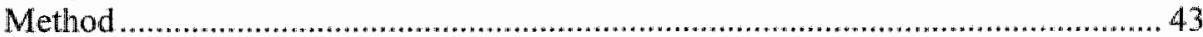

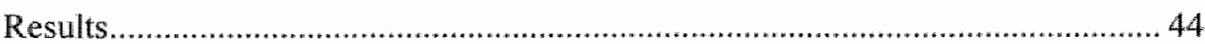

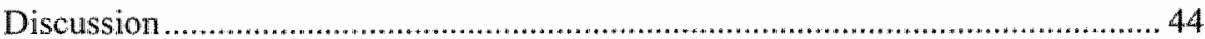

\section{PART II: DISSOCIATION, ATTENTIONAL CONTROL, AND} MEMORY

Chapter 4: Dissociative experiences in undergraduate students reflect lack of cognitive efficiency. 


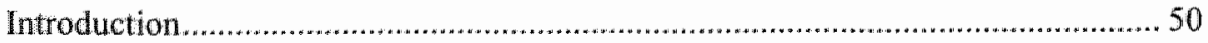

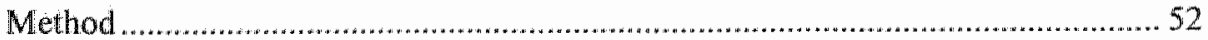

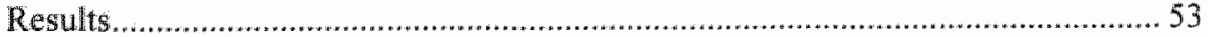

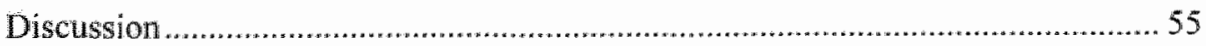

Chapter 5: Dissociation in undergraduate students: Disruptions in executive

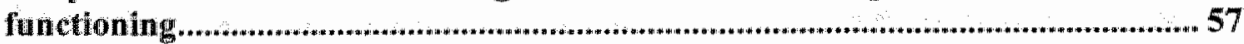

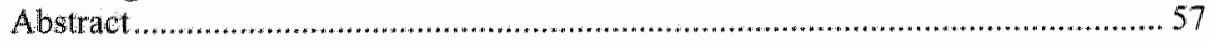

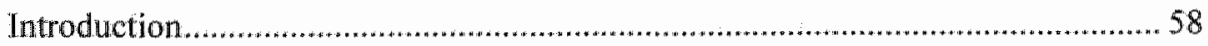

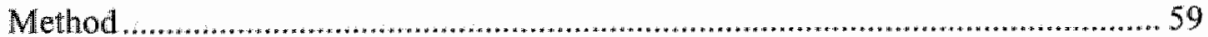

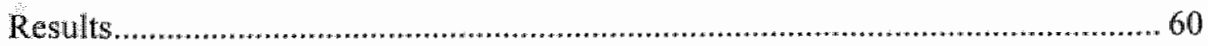

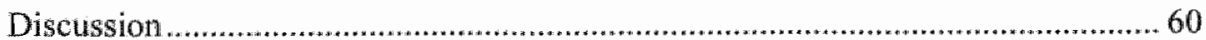

Chapter 6: First you find them, then you don't - Correlations between thought suppression and dissociation and their context sensitivity........................................63 63

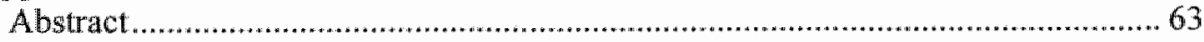

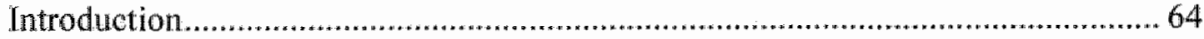

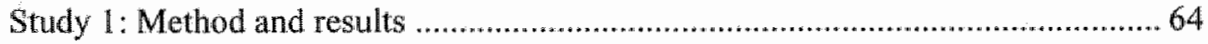

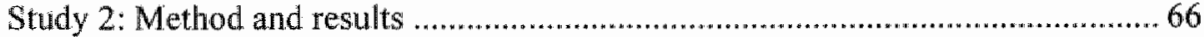

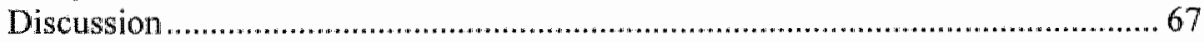

\section{PART III: DISSOCIATION, SLEEP, AND CORTICAL ACTIVITY}

Chapter 7: Subjective Sleep Experiences Are Related to Dissociation ...................73

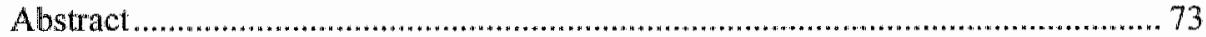

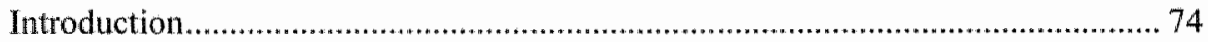

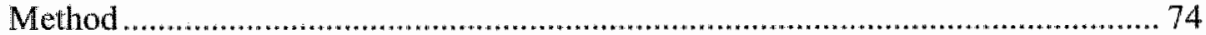

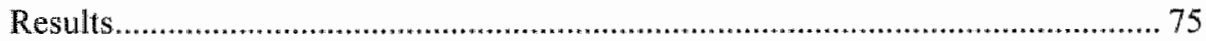

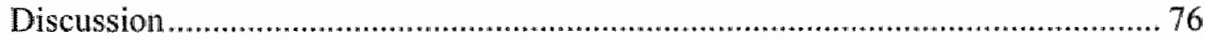

Chapter 8: Dreaming to reduce fantasy - Fantasy proneness, dissociation, and subjective sleep experiences .......................................................................................... 79

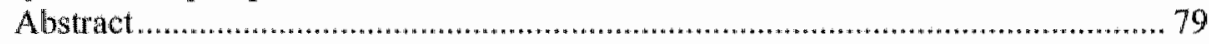

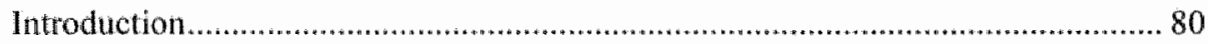

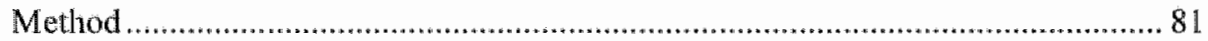

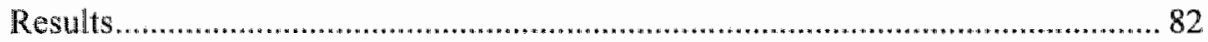

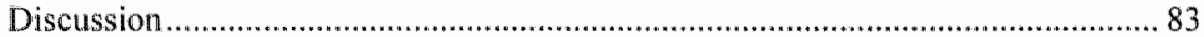

Chapter 9: Dissociation, resting EEG, and subjective sleep experiences in

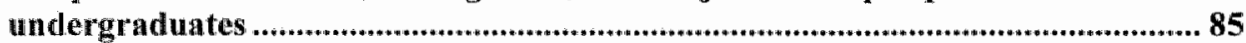

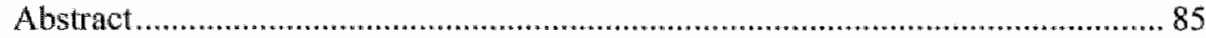

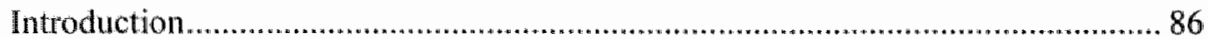

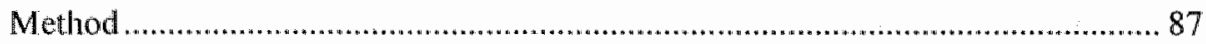

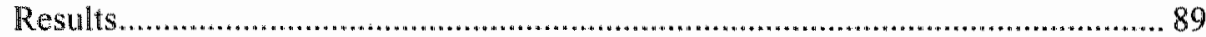

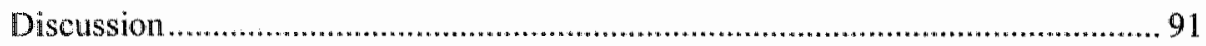


Dissociation: A defensive naneuver? ............................................................... 100

Future perspectives …........................................................................................... 101

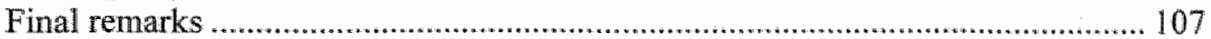

Submitted manuscripts 


\section{General introduction}

When ideas go unexamined and unchallenged for a long time ... they become mythological, and they become very, very powerful.

- Edgar Lawrence Doctorow 


\section{A typical case ${ }^{1}$}

The neighbors were suspicious since the first day the people next door moved in, but they failed to see something specific. If asked, they would tell that there was a strange family living next door. However, Pricilla, the oldest daughter of this family, behaved most weird. This girl could sit in the garden for hours, while reacting to absolutely nothing. Sometimes, when they met the girl in the mall, Priscilla seemed not to recognize them, while at other times, she reacted extremely enthusiastic.

However, suddenly the whole neighborhood understood what was going on. Pricilla was behaving maladjusted at school and therefore, she was sent to a psychiatrist. She was playing truant at school, but later she denied remembering anything of it at all. She told the psychiatrist about the tense relationship with her father. In addition, she told the psychiatrist that she could spend hours just staring and daydreaming. Besides, their conversation revealed that she sometimes felt very weird. Everything seems to happen subdued, as if things were not really happening. It felt more like a movie to her. Later she stated in vague terms that she had been raped by her nephew.

Based on what Pricilla had told him, the psychiatrist concluded that she was suffering from a dissociative disorder caused by the particularly traumatic incident of being raped by her nephew. He advised her to report the crime, which would be of great importance to resolve the trauma and as a consequence her dissociative disorder. During the subsequent lawsuit, the psychiatrist reported that Pricilla was suffering from a dissociative disorder, which is solely encountered in survivors of severe traumatic experiences, in her case rape. Therefore, her symptoms have had to be interpreted as a means of coping with these experiences and should be considered as additional evidence to bolster the credibility of her report.

\footnotetext{
${ }^{1}$ See for a simillar case, Case No. AU0582, Rechtbank Zutphen, 21 July 2004, available at: http://rechtspraak.nl.
} 


\section{Outline of the present thesis}

This case illustrates a recurrent theme both in the clinical literature (e.g., Gast, Rodewald, Nickel, \& Emrich, 2001; Gershuny \& Thayer, 1999), as well as in the legal domain. Both practitioners and researchers all too often assume that dissociative symptoms (and dissociative disorders in particular) are caused by exposure to traumatic events, such as childhood sexual abuse. These persistent dissociative symptoms should, according to the received view in the clinical literature, be regarded as the manifestation of a defense mechanism to withdraw yourself psychologically from the overwhelming impact of traumatic events.

The present dissertation empirically tests this theme, which is commonly voiced in the clinical literature. The first chapter reviews the literature on the relationship between persistent dissociative experiences and trauma. It highlights methodological shortcomings in past research and deduces hypotheses that flow from the assumption that these dissociative experiences are, indeed, due to a defensive mechanism. In addition, an alternative explanation, i.e., that dissociative symptoms might stem from a disrupted sleep-wake cycle, is considered. Both these aforementioned hypotheses and the alternative view concerning the etiology of dissociative experiences are investigated in the subsequent chapters.

Part I directly investigates the hypothesis that if dissociation would, indeed, possess defensive properties, heightened levels of dissociation should reduce physiological responsivity (i.e., emotional blunting) during psychological stress. This is accomplished by studying participants with differing frequencies of dissociative experiences using autonomic measures (i.e., skin conductance and cortisol response) during emotionally evoking events, such as an aversive video fragment or a stressful job interview.

The notion that dissociative experiences are the manifestation of a defense mechanism predicts specific aberrations in memory and attention which are addressed in part II. Notably, these experiences should be limited to a particular domain, specifically the domain of aversive stimuli, while not affecting the information processing of neutral stimuli.

Part III empirically tests an alternative explanation for the evolvement of dissociative symptoms. In particular, the relationship between dissociation and sleep experiences, such as nightmares and narcolepsy, is explored. Additionally, the neurophysiology of dissociation is investigated.

Finally, our findings from part I to III are integrated and their contribution to our understanding of dissociation is highlighted. Furthermore, attention is drawn to future research avenues. 


\section{Chapter 1: Dissociation and trauma: A review}

* Chapter 1 is an adjusted and translated version of the following articles: Giesbrecht, T., \& Merckelbach, H. (2005). Über die kausale Beziehung zwischen Dissoziation und Trauma: Ein kritischer Uberblick. Der Nervenarzt, 76, 20-27, and of Giesbrecht, T., \& Merckelbach, H. (2006). Dissociatieve Symptomen en Slaap. Tijdschrift voor Psychiatrie, 48, 207-215. 


\section{Introduction}

According to Bernstein and Putnam (1986, p.727), dissociation is "the lack of normal integration of thoughts, feelings, and experiences into the stream of consciousness and memory." These symptoms are commonly considered being the manifestation of an automatic psychological defense mechanism, employed by victims of traumatic experiences to withdraw themselves from the impact of these incidents (van IJzendoom \& Schuengel, 1996). However, while many authors regard symptoms of derealization, depersonalization, and psychogenic amnesia as core features of dissociation, the concept of dissociation is semantically open and still lacking a generally accepted definition. This led to a situation in which the term dissociation is also employed to describe various additional phenomena such as perception without awareness and hypnosis (Carderia, 1994).

At the end of the nineteenth century, Pierre Janet (1889) started to systematically investigate dissociative symptoms, thereby coining the term of psychological dissociation. In addition, Janet is considered the first scientist linking. dissociation to stress and psychological trauma (Gast et al., 2001). Originally, he regarded dissociation as a coping mechanism that an individual might use to reduce the impact of traumatic experiences. However, the use of dissociation as coping mechanism during a traumatic event can cause individuals to dissociate habitually even to minor stressors, which in turn leads to emotional constriction, causing various types of psychopathology (van der Kolk \& van der Hart, 1989). The idea that trauma directly causes dissociation is prominently present in the clinical literature (see for a critical review: Merckelbach \& Muris, 2001) and is often presented as an uncontroversial issue (Gast et al., 2001; Gershuny \& Thayer, 1999; Irvin, 1998).

The idea that trauma directly causes dissociation is critically evaluated in this chapter. Firstly, the primary measure of dissociation, the Dissociative Experiences Scale (DES; Bernstein \& Putnam, 1986) will be described. Next, studies investigating the relationship between dissociation and traumatic experiences will be discussed and their shortcomings will be pointed out. In addition, the overlap between dissociation and personality traits that might have confounded findings from many studies in this domain will be highlighted. Finally, an alternative model linking dissociative experiences to distuptions in circadian rhythms will be presented.

\section{The Dissociative Experiences Scale as a measure of dissociative experiences}

The official inclusion of dissociation in the Diagnostic and Statistical Manual of Mental Disorders III (DSM III; American Psychiatric Association, 1980) led to the development of the Dissociative Experiences Scale (DES; Bernstein \& Putnam, 1986). The DES is the standard instrument to quantify the frequency of dissociative symptoms (Merckelbach, Horselenberg, \& Schmidt, 2002) and has been used extensively in both clinical and non-clinical samples (van IJzendoorn \& Schuengel, 1996). Its widespread use is reflected by the fact that a computerized search in October 2005 using PsychINFO indicated that 190 studies have employed this measure since its development in 1986. This self-report measure quantifies the frequency with which respondents experience 28 dissociative phenomena in daily life. These phenomena 
include experiences of autobiographicall amnesia, derealization, depersonalization, and absorption and are measured on $100-\mathrm{mm}$ visual analogue scales. The respondents are asked to indicate how often (in percent of the time) they experiences particular dissociative symptoms. The DES total score consists of the arithmetic mean of all 28 items and can vary between 0 and 100 . Higher values imply an increased frequency of dissociative experiences, whereas values above 25 or 30 indicate potential psychopathology (Putnam, Carison, Ross, \& Anderson, 1996).

By means of a meta-analysis, van IJzendoorn and Schuengel (1996) underpinned the validity of the DES by investigating the relation between this instrument and various questionnaires, as well as semi-structured interviews. Their analysis revealed that the DES did not only correlate with other scales measuring related phenomena (Perceptual Aberration Scale, Questionnaire of Experiences of Dissociation, Bliss Scale, Dissociation Questionnaire), but even more so with the outcomes of the semi-structured interviews (Structured Clinical Interview for DSM III-R Dissociative Disorders, Dissociative Disorders Interview Schedule). Additionally, the DES was found to exhibit an excellent internal consistency (Cronbach's $\alpha=.93)$ and good temporal reliability with test-retest correlations ranging from .74 to .84 (Holtgraves \& Stockdale, 1997).

While the DES had been developed to measure an unidimensional dissociative construct, other researchers were quick to point out that the DES might consist of various subscales. A factor analysis by Carlson et al. (1991) yielded three factors, notably amnesia, absorption, and depersonalization. With respect to the factorial structure of the DES consensus has, yet, to be reached. While various studies report Carlson et al.'s three subscales (Ross, Ellason, \& Anderson, 1995; Sanders \& Green, 1994) or four factors (Ray \& Faith, 1994), others emphasize the unidimensionality of the DES. This unidimensionality of the DES is underpinned by Waller (1995) who reanalyzed the original Carlson et al. (1991) data and concludes that the three factor solution might be due to the skewed distribution of the DES with the three factor solution merely being a statistical artifact (for a recent contribution to this discussion, see Holmes et al., 2005).

In order to be able to discriminate between benign and pathological forms of dissociation, Waller, Putnam, and Carlson (1996) developed the DES-T. They extracted eight items from the DES measuring derealization and depersonalization, but excluded items tapping the less pathological features of dissociation, notably absorption. The probability that a respondent exhibits a pathological degree of dissociative experiences is calculated using a taxometric method.

As the DES is unsuitable for adolescents, Armstrong, Putnam, Carlson, Libero, and Smith (1997) developed the Adolescent Dissociative Experiences Scale (A-DES). This is a version of the DES that is adjusted to the cognitive capabilities of adolescents and consists of 30 questions with an 11-point scale. The internal consistency of the A-DES is high (Cronbach's $\alpha=.93$; Armstrong et al., 1997; Muris, Merckelbach, \& Peeters, 2003).

\section{The causal relation between dissociation and traumatic experiences}

The Diagnostic and Statistical Manual of Mental Disorders (DSM IV; American Psychiatric Association, 1994) defines an event as a trauma, when the individual 
witnesses or is confronted with death, the threat of death, or injury, i.e., a threat to the individual's or other people's bodily integrity. In addition, the individual's reaction has to be one of horror, fear, or helplessiness. This definition is the consequence of various author"s critique (Gershuny \& Thayer, 1999), implying that the third version of the Diagnostic and Statistical Manual of Mental Disorders (DSM III; American Psychiatric Association, 1980) focuses on the exceptionality of the event to an unreasonable extent. According to these critics, this prerequisite is in stark contrast to the prevalence of traumatic experiences, which varies from 40 to $72 \%$ in the general population (Gershuny \& Thayer, 1999). Consequently, the definition of the DSM IV focuses more on the subjective reaction of the individual than previous definitions, thereby allowing for the possibility that different persons can experience the same event as more or less traumatic (Gershuny \& Thayer, 1999).

Proponents of the causal link between trauma and dissociation frequently draw their evidence from positive correlations between measures of dissociation and retrospective self-report of traumatic events. These correlations are found in both clinical and non-clinical populations. Various clinical studies that are directed at the link between trauma and dissociation will be described, first, as well as the disadvantages of clinical samples in the study of this particular relationship.

The link between their own Sexual Assault Questionnaire (SAQ) and the DES was investigated by Zlotnick et al. (1996). The SAQ quantifies various properties of sexual assault, including age at onset of the abuse, frequency, and identity of perpetrator. Their study yielded a significant correlation of 40 between the SAQ and the DES in a sample of 103 self-mutilators and 45 non-mutilators. It shoulld, however, not go unnoted that the authors state that both the reliability and the validity of the instrument are dubious since studies investigating the psychometric merits of the SAQ are lacking.

In another study, Nash, Hulsey, Sexton, Harralson, and Lambert (1993) also underpinned the relationship between dissociative experiences and self-reports of abuse in a sample of 105 abused and non-abused women. They noted that compared to controls, women with a history of self-reported sexual abuse exhibited significantly elevated scores on both the Indiana Dissociation Scale and the Dissociation Content Scale. At first sight, their finding nicely fits the idea that traumatic events lead to an increase in dissociative symptomatology. However, an interesting aspect of this study is that these findings were mediated by a third factor, as, when family dysfunctioning was held constant statistically, differences in level of dissociation disappeared.

In a related study, Sanders and Giolas (1991) scrutinized the relationship between the DES and childhood sexual abuse by using a questionnaire developed by themselves in a sample of 47 psychologically disturbed adolescents that were institutionalized in a private mental hospital. Their questionnaire quantified negative experiences in four different domains: physical maltreatment, physical abuse, sexual abuse, and neglect. Significant correlations emerged between the DES and their sexual abuse questionnaire's four factors ranging from .26 to .50 and a correlation of .44 between the DES and the mean score of their questionnaire.

However, all these findings have to be interpreted with caution, as one major disadvantage of using clinical samples is that these samples might not be representative for a population of individuals having suffered abuse and might be even less representative for the general population. As dissociative experiences are often considered being continuously distributed and high in frequency even in the general population, using clinical samples might lead to distortions and inflations of findings for 
the following two reasons. Firstly, patients might be more willing to actively look for a justification of their problems and might therefore be more tempted to report events that might be classified as abuse. This distortion of the data is often amplified by the fact that members of dysfunctional families are overrepresented in these samples (Rind, Tromovitch, \& Bauserman, 1998). Secondly, one might expect that individuals with a history of abuse in combination with dissociative experiences might more readily search help than persons with a history of abuse but without dissociative symptoms (Pope \& Hudson, 1995).

Moreover even the treatment of sexual abuse itself can lead to an increase in dissociative symptoms, thereby inflating correlations artificially. Winkielman, Schwarz, and Belli (1998), for example, compared participants who had to recall three events from their adolescence with others that had to recall nine events. Their study indicates that, ironically, participants, who had to bring to mind more autobiographical memories, were more likely to experience a feeling of having forgotten substantial part of their adolescence, as compared to participants who had to recollect less autobiographical memories. Likewise, psychotherapy involves retrieval of past experiences and could therefore lead to a feeling of dissociative amnesia. To circumvent these problems, non-clinical populations should be preferred when investigating the relation between trauma and dissociation.

Various studies investigated the relation between retrospective self-report of trauma and dissociation in non-clinical samples (e.g., Dalenberg \& Palesh, 2004; Irwin, 1999; Martinez Taboas \& Bernal, 2000; Zoroglu et al., 2003). For example, Sandberg and Lynn (1992) compared 33 female students with a high frequency of dissociative experiences, as measured by the DES, to the same number of students reporting a low frequency of these experiences with respect to retrospective self-report of psychic, physical, and sexual abuse. Their study showed that the frequency of dissociative experiences is related to their measure of abuse even when the tendency to answer socially desirable is partialled out.

The aforementioned or similar studies showing that dissociation is related to retrospective self-reports of trauma are regularly cited as evidence for a direct causal relationship between trauma and dissociation. However, all of them possess some common shortcomings. Firstly, findings are difficult to interpret and to compare, as often unstandardized, unvalidated questionnaires, with unknown reliability, were used in order to quantify traumatic experiences (Irvin, 1998; Sanders \& Giolas, 1991; Zlotrick et al., 1996). Secondly, some studies employ non-representative samples which might result in artificial distortion and exaggeration of relations.

Two other studies (Johnson, Edman, \& Danko, 1995; Merckelbach \& Jelicic, 2004) demonstrate another serious threat to the validity of studies investigating the relationship between trauma and dissociation by means of retrospective self-report, which is a positive response bias that is associated with heightened levels of dissociation. Johnson, Edman, and Danko (1995) studied the relationship between trauma and dissociation using the Bad Things Scale (BTS), a questionnaire, that retrospectively quantifies various negative experiences, ranging from serious ("I have been sexually abused by a family member.") to insignificant events ("I have been sold defective products."). These experiences were measured on a 4-point scale. In a sample of 94 students, these researchers found a correlation of . 42 between the BTS and the DES. In the light of the trauma-dissociation doctrine, one might be tempted to interpret these finding as lending support to the view that trauma is an important antecedent to dissociation. Interestingly, Johnson et al. (1995) repeated their analysis, while excluding 
all items that refer to abuse. As the trauma-dissociation view assumes that the exposure to severe trauma leads to dissociation, one would expect that the relation between the DES and the BTS without the abuse items would be low. The opposite was true. Unexpectedly, the relation between the DES and the BTS remained intact. In a related study, Merckelbach and Jelicic (2004) investigated the origin of the link between dissociation and retrospective self-report of trauma in two samples of 43 and $\$ 27$ students. Their study indicated that the trauma-dissociation link is carried by high dissociators' positive response tendency to vague trauma-items and items that ask for beliefs or opinions (e.g., "Do you recall an adult looking at you when you were less than fully dressed in a way that you suspect might have had a kind of sexual meaning for that adult?"). It is worthy of note that this positive bias was not present for specific traumaitems (e.g., "Do you remember any kind of penetration by an adult?").

This positive response tendency, which is associated with heightened levels of dissociation, might confound all studies that rely on retrospective self-report of traumatic experiences. This problem gets even more obvious when one looks at another facet of the Sanders and Giolas (1991) study. At first glance, their finding that dissociation is related to retrospective self-report of trauma appears to support the trauma-dissociation view. However, in addition to retrospective self-report measures of traumatic events, hospital records of their participants were scored in this study by a "blind" rater for indications of trauma. This procedure yielded a slightly negative correllation between ratings of traumatic experiences based on hospital records and dissociation $(r=-.21, P=.10)$. This finding supports the notion that dissociation is related to retrospective self-reports of traumatic experiences and especially to vague trauma items or items that ask for beliefs or opinions (Merckelbach \& Jelicic, 2004). However, dissociation appears to be unrelated to objective indices of such experiences. This finding is backed by a study of Cima, Merckelbach, Klein, Schellbach-Matties, and Kremer (2001), who also failed to find any relationship between dissociative symptoms and objective indicators of childhood traumatic experiences in a sample of 30 forensic psychiatric patients.

One possibility to circumvent some of the pitfalls of retrospective self-report of traumatic events is to employ prospective studies. Interestingly, there are two prospective studies, which tried to elucidate the developmental pathway to dissociation. Macfie, Cicchetti, and Toth (2001) were able to examine dissociation in a group of maltreated $(n=45)$ and a group of non-maltreated children $(n=33)$ between the age of 3 and 4. Their study showed higher levels of observer-rated dissociation in maltreated children. In another prospective longitudinal study, Ogawa, Sroufe, Weinfield, Carlson, and Egeland (1997) studied the development of dissociation in a sample of children, who were considered high-risk for poor developmental outcomes at birth due to poverty. They followed their participants for an impressive period of 19 years. During the first set of these sessions, dissociation was quantified by observer ratings, but later by self-report. This study yielded modest correlations between dissociation and trauma, which are of a similar magnitude as the ones based on retrospective self-report of trauma, that were discussed earlier. In addition, this study generated some other interesting findings, which might prove problematic for the idea that there exists a direct causal relation between dissociation and trauma. Firstly, the authors conclude that "it is not possible to say that earlier onsets of trauma lead to higher levels of dissociation" ( $p$. 865), which is in contrast to the commonly held assumption that vulnerability to trauma is highest in early childhood. Secondly, another, maybe even more stunning, finding is the fact that early sexual abuse did not predict dissociation at the end of the study (when 
participants were 19 years old). This is worthy of note, as it was only during the 19 years follow-up measurement that the DES was employed to measure dissociative tendencies. Remarkably, this last measurement of dissociation was unrelated to all other measurements dissociation, which is in stark contrast to the stability of dissociative experiences reported elsewhere (van Uzendoorn \& Schuengel, 1996). As a result, it is uncertain, what the prior measurements of dissociation were actually measuring.

Apart from these problematic findings, some methodological shortcomings deserve consideration. Firstly, similar to the clinical studies mentioned earlier, these two longitudinal studies did employ non-representative samples, which might have inflated their findings. Relevant to this issue is a meta-analysis by Rind et al. (1998) that indicates that in the general population the relationship between retrospective self-report of trauma and dissociation is weak (effect size: $r=.07$ ) and is confounded by family environment, which explains considerably more variance. Secondly, a large community sample study $(n=1000)$ highlights the importance of controlling for psychopathology, since the authors (Mulder, Beautrais, Joyce, \& Fergusson, 1998, p. 809) summarize their findings as follows:

"Any causal influence of childhood sexual abuse on dissociation is likely to be indirect and mediated by more general linkages between childhood sexual abuse and ristss of mental disorder."

In sum, the aforementioned studies show that there is a relation between dissociation on the one hand and retrospective self-report of traumatic events on the other. However, none of the aforementioned studies allows the conclusion that there is direct causal relationship between actual trauma and dissociation. This is due to the fact that, dissociative tendencies go along with a positive response bias (Johnson et al., 1995; Merckelbach \& Jelicic, 2004), while studies based on objective evidence failed to substantiate the relationship between trauma and dissociation. Moreover, most research did not control for other confounding factors such as family pathology and psychopathology.

\section{Dissociative experiences and other personality traits}

If worth asking why dissociative tendencies are linked to this positive response tendency, which confounds studies that make use of retrospective self-report measures of trauma. A number of personality traits that overlap with dissociation, but have not been mentioned, yet, might shed light on this matter. These traits include fantasy proneness, suggestibility, and the susceptibility to cognitive failures.

Fantasy, claydreaming, and imagination are essential for the healthy psychological functioning. The inability to control these processes, however, can lead to psychological problems (Rauschenberger \& Lymn, 1995). Nevertheless, most individuals, who fantasize extensively, exhibit no signs of psychopathology (Merckelbach \& Muris, 2001). Various studies established the relationship between dissociative experiences and fantasy proneness. Rauschenberger and Lynn (1995) compared 26 participants, who reporting fantasizing extensively, with the same number of participants, who fantasized modestly, as quantified by the Inventory of Childhood Memories and Imaginings (ICMS). The authors found that undividuals, who fantasize more, also dissociate more frequently. Silva and Kirsch (1992) also established a correlation of 42 between the ICMS and the DES. Other studies obtained comparable results with correlations ranging from .53 to .58 (Merckelbach et al., 2002; Merckelbach, Muris, \& Rassin, 1999; Merckelbach, Muris, Rassin, \& Horselenberg, 
$2000 \mathrm{~b})$. Importantly, the conmection between dissociative experiences and fantasy proneness is not limited to non-clinical samples (Merckelbach, à Campo, Hardy, \& Giesbrecht, 2005; Pekala et al., 1999-2000). Pekala et al. (1999-2000) found a significant correlation of .41 between these two personality traits in a sample of 1229 drug addicts, while Merckelbach et al. ${ }^{3} s$ (2005) study yielded a correlation of .55 in a mixed sample of psychiatric patients.

The proneness of individuals, who dissociate frequently, to engage in fantasizing, can have profound consequences for our understanding of the causes of dissociative experiences. More specifically, this promeness migh endanger the validity of the questionnaires measuring the occurrence of trauma retrospectively. Fantasy proneness might influence the results in two ways. Firstly, fantasizers might exhibit a tendency to confuse imagined events and factual autobiographical memories. This would be a reality monitoring error. Secondlly, these individuals might employ a more liberal criterion to report an experience as being genuine, thus, exhibiting a tendency to confabulate.

Merckelbach et al. (2000a) studied the relation between the DES, confabulation, and reality monitoring errors in two experiments. In the first experiment, particupants watched a series of 40 slides. Twenty were photos of common abjects or situations, whereas the other 20 consisted of a short paragraph, describing an object or a situation. Ten of the latter were related to slides that were shown previously. Fifteen minutes later, a surprise recognition task followed. The participants were asked whether they had seen the slide in question previously. Confirmatory answers were followed by the question whether the slide had been presented as a picture, a paragraph, or both. This task taps two kinds of errors. Firstly, participants can mistakenly indicate having seen a photo while the original slide was a paragraph or vice versa. This would be a reality monitoring error. Secondly, participants may confabulate, thus indicating having seen a slide that was not present during the study stage. This experiment noted a significant correlation of .39 between the DES and the tendency to confabulate, but no relationship between dissociation and reality monitoring errors.

More evidence for the idea that respondents that are high on dissociation exhibit an increased tendency to confabullate comes from a second study. Merckelbach et al. (2000a) asked 70 participants to complete the Life Events Inventory (LEI). This is a questionnaire consisting of 60 items describing 20 negative, 20 positive, and 20 neutral events. These items are detailed descriptions of specific experiences (e.g." "f went to Disney Land with my school."). The respondent is asked to indicate how certain they are having experienced these particular events on a 7-point scale. It goes without saying that some respondents will have actually experienced some of the events. If a respondent, however, indicates having experienced most or all events, which is highly improbable, this indicates a tendency to confabulate. In this study, a significant correlation of .39 between the DES and the LEI emerged. This correlation, however, disappears when the influence of fantasy proneness was partialled out. This study underpins the idea that individuals with an extensive amount of dissociative experiences, more readily endorse descriptions of events they have never experienced.

Another relevant personality trait is interrogative suggestibility, which is the extent to which, within a closed social interaction, individuals accept messages embedded in questions, which later influence their behavior. Individuals differ widely in the tendency to adjust their behavior as a result of misleading information or

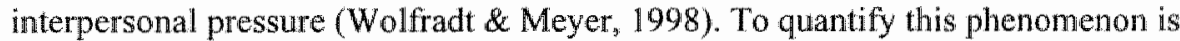
essential, especially in the legal domain. With this in mind, Gudjonsson (1984) 
developed the Gudjonsson Suggestibility Scalle (GSS), which measures suggestibility on two scales. The Yield-scale quantifies the extent to which answers are shaped by misleading questions. The Shift-scale measures whether the individual is prone to changing the answer, when prompted by the interviewer. The sum of both scales represent the GSS total score. Wolfradt and Meyer (1998) found significant correlations of .53 between the DES and the Yield-scale and .44 between the DES and the ShiftScale. In addition, the DES and the GSS total score correllated.54. Merckelbach et al. (2000b) found a weaker relation. In their study the DES and the GSS total score and the DES and the Yield-score correlated .37 and .29 , respectively, whereas the correlation between the DES and the Shift-scale was non-significant. The significant correlations between DES and suggestibility indices, however, disappeared when the influence of cognitive failures (see below) was statistically corrected for. Nevertheless, the conclusion that individuals high on dissociation are also higher on suggestibility remains valid.

Besides to being more prone to fantasy and suggestion, individuals high on dissociation also say they commit more cognitive failures, as measured by the Cognitive Failures Questionnaire (CFQ; Broadbent, Cooper, Fitzgerald, \& Parkes, 1982). This questionnaire asks respondents to indicate on a 5-point scale how frequent they commit everyday cognitive failures, such as forgetting names, missing signs on the road, or being distracted (Boomsma, 1998). The correlation between the DES and the CFQ ranges from .43 to .51 (Merckelbach et al., 2002; Merckelbach et al., 1999).

Furthermore, it should not remain unmentioned that dissociation modestly correlates with other psychopathological syndromes, such as depression, as measured with Beck's Depression Inventory (van IJzendoorn \& Schuengel, 1996) and schizotypal traits (e.g., Pope \& Kwapil, 2000). Most studies, however, do not control for psychopathology as a cofounder.

\section{Heritability of dissociation, cognitive failures, and fantasy proneness}

The previous paragraph highlighted the relationship between dissociation and three personality traits, notably fantasy proneness, suggestibility and the susceptibility to cognitive failures. We drew attention to the finding that individuals who dissociate more frequently also fantasize more. This, in turn, leads to more confabulations, possibly resulting in pseudo-memories (Merckelbach et al., 2000a). In addition, dissociation also overlaps with susceptibility to cognitive failures. This might function as a confounder in research that attempts to retrospectively exposure to traumatic experiences, as individuals who report many cognitive failures, are more prone to mistrust their memory. This would make them more susceptible to suggestive post-hoc information. It: can, therefore, not be precluded that a combination of the three personality traits fantasy proneness and the susceptibility to cognitive failures might add to the retrospective report of traumatic experiences.

Merckelbach et al. (2002) investigated the idea that the relationship between self-reported trauma and dissociation is due to individual differences in cognitive failures and fantasy proneness. This was done by using structural equation modeling in a non-clinical sample of 109 undergraduates. Two contradictory models were tested. Model I was the classical trauma-dissociation model, which assumes that trauma precedes dissociation, which in turn leads to an increase in fantasy proneness and 
cognitive failures. In contrast, model 2 presumes that dissociation is the starting point, leading to fantasy proneness and cognitive failures. The last two should, in this model, contribute to retrospective reports of traumatic experiences. The analysis underpinned both models, thereby not ruling out the possibility that fantasy proneness and cognitive failures lead to an over-reporting of trauma.

This alternative model, however, raises the question of the origins of dissociative experiences. Research investigating the heritability of cognitive failures and fantasy proneness might contribute to solving this problem. In her situdy on monozygotic and dizygotic twins, Boomsma (1998) could, indeed, show that a "a substantial part of the inter-individual variation in everyday cognitive failures in memory, perception and motor control can be attributed to genetic factors." The proneness to fantasize, which overlaps with dissociation, can also be traced back to genetic factors. Fantasy proneness is regarded as a manifestation of the superordinate personality trait "openness to experience". This trait has a substantial heritability (Bergeman et al., 1993), which should, at least in part, modulate fantasy proneness. Using twin register data, Lang, Paris, Zweig-Frank, and Livesley (1998) directly investigated the heritability of dissociative experiences. They report that about half of the variability in dissociative experiences can be attributed to genetic factors. The evidence concerning the genetic predisposition of dissociative experiences is, however, mixed, as another study (Waller \& Ross, 1997) reports no genetic influence. The most recent study looked at children and adolescents and this study lends support to the notion of a genetic predisposition of dissociation (Becker-Blease et al., 2004). If dissociation would, indeed, possess a strong genetic basis, this would be in stark contrast to the postulated traumatic etiology of dissociative experiences.

\section{The defensive properties of dissociation}

In the previous sections we have presented two contrasting views concerning the relationship between dissociation and trauma. The first one, termed the trauma-dissociation view, is most prominently represented in the clinical literature and states that traumatic experiences are an important developmental antecedent of dissociative tendencies (e.g., Classen, Koopman, \& Spiegel, 1993; Holmes et al., 2005; Kersting et al., 2003; Reinders et al., 2003). In contrast, the alternative view suggests that dissociation overlaps with fantasy proneness and cognitive failures, which in turn lead to an inflation of the relation between dissociation and self-reported trauma (e.g., Merckelbach et al., 2002; Merckelbach \& Muris, 2001). According to this view, trauma is not necessary for the evolvement of dissociation.

By now, it should be plain to the reader that the direct causal relationship between trauma and dissociation is difficult and cumbersome to examine. The only appropriate way to test it would be by employing large, longitudinal studies that follow participants with and without traumatic experiences. However, there exists an alternative and more practical possibility to investigate the causal relation between dissociation and trauma which will be described below. This line of reasoning is based on the core assumption of the trauma-dissociation view. As only in the traumadissociation view, dissociation serves as a defense mechanism, this allows us to make specific predictions concerning the effect of heightened levels of dissociation on emotion, memory, and attention.

One assumption of the trauma-dissociation view is that the defensive properties of dissociation serve to regulate the impact of owerwhelming emotions. This is 
emphasized by Gershuny and Thayer $(1999$, p. 647) who state that "when emotional pain is acute and deemed unbearable by the sufferer, dissociation may be called upon as means of escape." These dissociative symptoms should manifests themselves in the form of "freezing, analgesia, and emotional numbing [which] are elicited automatically" (Nijenhuis, Spinhoven, Vanderlinden, van Dyck, \& van der Hart, 1998, p. 65).

Anecdotal evidence for decreased or absent pain sensation during disaster underpins this view (Spiegel \& Cardena, 1991). In line with the aforementioned assertions, Williams, Haines, and Sale (2003, p. 102) hypothesize that

"If dissociative experiences associared with DID are a form of coping, it should follow that the experience of dissociation as part of DID should produce a somatic and psychic anxiety reduction that form the basis of the protection of the individual that dissociation episodes allow."

Thus, if dissociation is a defensive mechanism, one would expect high dissociators to exhibit diminished pychophysiological responsivity to aversive stimuli (i.e., emotional blunting; Sierra \& Berrios, 1998) due to their assumed abillity to withdraw themselves psychologically from emotional events (Markowitsch, 2003).

Another core assumption of the trauma-dissociation view can be traced back to Pierre Janet who stressed the importance of disturbance in memory for emotional events due to dissociation (van der Kolk \& van der Hart, 1989). This idea is rephrased by Bernstein and Putnam (1986) who highlight the importance of both disruptions of memory and attention in their definition of dissociation. There is broad consensus in the clinical literature that deficits in memory function (e.g., compartmentalization, psychogenic amnesia) should be considered core features of dissociation if dissociation would serve as a defensive mechanism (Ehlers \& Clark, 2000; Holmes et al., 2005; van der Kolk \& Fisler, 1995).

The detrimental effects of dissociation on attention and memory should stem from disturbances of information processing (Spiegel \& Cardena, 1991; van der Kolk \& Fisler, 1995) as "victims of a psychotraumatic event may protect themselves against the overwhelming exposure of threatening stimuli by inhibiting information processing" (Ladwig et al., 2002, p. 242). Thus, "dissociation may impede the elaboration of the trauma memory and its integration into the autobiographical knowledge base" (Ehlers \& Clark, 2000, p. 330). Eminent to the idea that dissociative symptoms, such as psychogenic amnesia and derealization, serve the function to reduce awareness during trauma, is the prediction that individuals with heightened levels of dissociation should be accompanied by slower processing of threat related information. Conversely, Spiegel and Cardena (1991, p. 367) state that dissociation "may influence ongoing experience and performance, either through priming or through increase or reduction of interference".

Thus, there is broad consensus concerning the profound influence of dissociation on attention and memory (Brewin \& Saunders, 2001; Cardeña, 1994; Classen et al., 1993; Holmes, Brewin, \& Hennessy, 2004; Spiegel \& Cardena, 1991) and the importance of exploring information processing characteristics that go along with heightened levels of dissociation (e.g., Dorahy, 2001), but there is some debate on the direction of the effect.

Few studies have followed that lead. A noteworthy exception are a series of studies by Freyd and colleagues (DePrince \& Freyd, 1999, 2004; Freyd, Martorello, Alvardo, Hayes, \& Christman, 1998), which only partially lend support to the notion that heightened levels of dissociation are solely related to deviations in the deployment of attention in response to aversive stimuli. Interestingly, Freyd et al. (1998) found that 
high dissociators showed more interference during the standard Stroop color naming task (1935), while being at advantage during divided attention conditions (DePrince \& Freyd, 1999). In addition, high dissociators remembered fewer aversive and more meutral stimulus words than low dissociators. The last findings can be interpreted in terms of the defensive properties of dissociations. The former findings, however, cannot, as they are not related to one specific stimulus domain (i.e, aversive stimuli).

In sum, our reading of the literature led us to conclude that if dissociative experiences were the manifestation of a defense mechanism, heightened levels of dissociation should result in deviations in information processing of aversive stimuli, but not neutral ones. This influence on information processing should in turn cause another core feature of dissociation, dissociative amnesia, manifesting itself as omission errors in memory. Furthermore, individuals with heightened levels of dissociation should exhibit attenuated psychophysiological responsivity to aversive stimuli, as compared to indiwiduals with a low frequency of dissociative experiences. In combination, these predictions can be used to indirectly test the causal relation between trauma and dissociation.

\section{Dissociation and sleep}

Another alternative explanation for the evolvement of dissociation has, until now, received surprisingly little attention. This is the influence of individual difference in sleeping behavior on dissociative tendencies. Individuals differ widely in both sleep quality and quantity, but also in whether they can remember their dreams. The dreamlike character of dissociative experiences seems to hint at a possible relationship between sleep-related experiences and dissociative experiences.

Watson (2001) investigated this matter using the lowa Sleep Experiences Survey (ISES). This questionnaire requires participants to indicate the frequency of various sleep- and dream-related experiences. The 18 items consist of questions such as "I can remember my dreams." and "I dream that I am falling." In two studies, Watson found significant correlations between the DES and the ISES of .53 and .54.

These findings indicate that individuals who dissociate more frequently, also exhibit a heightened frequency of various sleep experiences, such as nightmares or narcolepsy. This is important, as there is extensive evidence showing that sleep deprivation has profound effects on performance and physiological alertness (Jewett, Dijk, Kronauer, \& Dinges, 1999). Thus, a disrupted sleep-wake cycle would lead to an increment of cognitive failures. In addition, there is evidence indicating that sleep deprivation profoundly increases suggestibility (Blagrove, 1996).

Besides the influence of the sleep-wake cycle on cognitive failures, fantasy proneness could possibly also be influenced by the disruptions in sleep. One postulated function of the Rapid-Eye-Movement (REM) sleep is to increase the efficiency of the brain. Evidence for this comes from two sources. Firstly, the enchidna and various dolphins have relatively large brains, but they display no REM sleep. This signifies that the REM sleep might serve to store information more effectively in the brain. Secondly, research using neural nets illustrates that random stimulation originating from the brainstem during REM sleep might serve to prevent confusion between different information. Or as Crick and Mitchison (p. 150, 1995) stated: "We dream to reduce fantasy".

Disruptions in sleep might, thus, not only be related to cognitive failures, but possibly also to fantasy proneness. This means that the overlap between dissociation 
and sleep might be modulated by the influence of sleep on both cognitive failures and fantasy proneness. It should, however, not remain unmentioned that massive traumatic experiences lead in nearly all cases to sleeping problems. Harvey, Jones, and Schmidt (2003), for example, consider the latter as a typical reaction to traumatic experiences.

\section{Sleep experiences as an alternative explanation for the development of dissociative experiences}

The relevance of Watson's $(2001 ; 2003 \mathrm{~b})$ findings is that they offer an alternative for the popular opinion that dissociative experiences are the manifestation of a defense mechanism. This popular view lacks a proper empirical foundation (Kihlstrom, 2005; Merckelbach \& Muris, 2001). However, the lack of such a foundation does not necessarily render this opinion wrong. Above, we explained that the direct causal relationship between trauma and dissociation is difficult to examine and presented an altemative way to scrutinize this causal relationship. This alternative approach relies on the assumption that dissociative experiences are the manifestation of a defense mechanism.

Part I and II of this dissertation are dedicated to studies that critically evaluate one or more of these assumptions flowing from the idea that dissociative experiences possess defensive properties. Part I consists of studies that investigate the influence of dissociative tendencies on physiological reactivity. More specifically, the question is addressed whether dissociation goes along with emotional blunting during psychological stress. Part II, on the other hand, assesses whether dissociation is indeed, as is often assumed, related to deviations in cognitive processes within one certain domain of stimulus valence (i.e., aversive stimuli). The contrasting hypothesis, that dissociative experiences are related to a global deficit, as opposed to a selective one, is also considered. Conversely, part III describes studies that explore an alternative to the trauma dissociation view. This alternative view hypothesizes that disruptions in the sleep-cycle give rise to dissociative experiences ${ }^{2}$.

We would like to note that a different line of research is directed at the relation between acute dissociative symptoms during traumatic experiences and postraumatic stress disorder. We solely focused in our studies on the relation between trait dissociation and trammatic experiences and therefore suggest to readers interested in the relevance of peri-traumatic dissociation during traumatic experiences, such as torture or traffic accidents, the review article of Candel and Merckelbach (2004). 


\section{Part I: Dissociation and emotion}

Dissociation appears to be the mechanism by which intense sensory and emotional experiences are disconnected from the social domain of language and memory, the internal mechanism by which terrorized people are silenced.

- Judith Herman 


\section{Chapter 2: Dissociation, memory commission errors, and heightened autonomic reactivity*}

\section{Abstract}

If dissociative symptoms were manifestations of a psychological defense mechanism, one would expect that individuals, who experience such symptoms more frequently than others, should react with lower physiological arousal and memory omissions to emotionally provocative material. The current study tested this assumption in a sample of undergraduates. Sixty-two undergraduate students viewed a highly emotional video fragment. Pearson product-moment correlations were calculated between dissociation (as indexed by the Dissociative Experiences Scale) and all dependent measures. High dissociators exhibited raised skin conductance responses to the fragment. Memory for the video fragment was then tested. While omission errors were unrelated to dissociation, high dissociators exhibited a tendency to produce commission ertors. "This could not be explained by a reduced working memory capacity. However, fantasy proneness was found to be related to high dissociators' commission errors. Thus, it seems that a pattern of heightened emotional reactivity and commission errors is typical for people with raised dissociation scores. This pattern is difficult to reconcile with the defensive function ascribed to dissociation.

* Giesbrecht, T., Geraerts, E., \& Merckelbach, H. (submitted). Dissociation, memory commission errors, and heightened autonomic reactivity. 


\section{Introduction}

According to Bernstein and Putnam (1986, p. 727), dissociation is "a lack of normal integration of thoughts, feelings, and experiences into the stream of consciousness and memory". Many authors believe that it "functions to fragmentize, derealize, and depersonalize traumatic experiences" (Markowitsch, 2003, p. S133). Dissociation can be conceptualized as forming a continum that ranges from minor dissociation of everyday life to major forms of psychopathology (Fischer \& Elnitsky, 1990).

Historically, dissociation has been considered a defense mechanism that enables the individual to withdraw psychologically from the impact of overwhelming traumatic events (Gershuny \& Thayer, 1999). This view dates back to $19^{\text {th }}$ century psychologist Piere Janet (1894) who coined the term of dissociation and emphasized its role as a defensive maneuver in response to psychological trauma. A habitual tendency to dissociate would, however, promote psychopathology (Hacking, 1995). Following this tradition, recent clinical literature emphasizes the causal relationship between dissociation and trauma (Gast et al.., 2001; Gershuny \& Thayer, 1999; Irvin, 1998).

While the view that dissociation is caused by exposure to tratmatic events is widely accepted, empirical studies providing unequivocal support for this causal direction are still lacking. For example, Zlotnick, Shea, Pearlstein, Simpson, Costello, and Begin (1996) found a correlation of 40 between dissociation, as measured with the DES, and their own Sexual Assault Questionnaire (SAQ). Yet, the authors admit that the validity and the reliability of the SAQ are unknown. Similarly, Nash, Hulsey, Sexton, Harralson, and Lambert (1993) investigated the link between trauma and dissociation in a sample of 105 women. They found that participants with a selfreported history of sexual abuse endorsed significantly more dissociative symptoms than women without such history. However, this connection disappeared when a measure of family pathology was entered as covariate in the analysis. This suggests that the connection between trauma and dissociation might be less straightforward than is often thought. This point is emphasized by Mulder, Beautrais, Joyce, and Fergusson (1998, p. 809), who summarized their findings in a large community study $(n=1000)$ as follows:

"Any causal influence of childhood sexial abuse on dissociation is likely to be indirect and mediated by more general linkages between childhood sexual abuse and risks of mental disorder."

Another study yielding problematic results for the trauma-dissociation hypothesis is that by Sanders and Giolas (1991). These authors found retrospective selfreports of traumatic experiences and dissociative symptoms to be modestly correlated in a sample of adolescent psychiatric patients. Curiously, when hospital records were scored by a "blind" rater for indications of trauma, a slightiy negative correlation emerged between ratings of traumatic experiences based on hospital records and dissociation. This suggests that dissociation is related to retrospective self-reports of traumatic experiences, while being unrelated to more objective and specific indices of such experiences. Recently, Merckelbach and Jelicic (2004) offered an explanation for this phenomenon. In two samples of undergraduate students $(n=43$ and $n=127)$, they found that dissociative experiences are related to endorsement of vague, but not specific trauma items. This response tendency might also have played a role in other studies that found a link between dissociation and trauma as measured by retrospective self-report scales of traumatic experiences. 
Mild dissociative symptoms are rather common in the general population, with $80 \%$ to $90 \%$ of the respondents indicating that they sometimes experience these symptoms (Gershumy \& Thayer, 1999). This is difficult to reconcile with the alleged traumatic etiology of dissociative symptoms, which implies that they should have a lower base rate. In addition, Lang, Paris, Zweig-Frank, \& Livesley (1998) investigated the influence of heritability on dissociative experiences using a twin study. They report that about half of the variability in dissociative experiences can be attributed to heritability. The evidence concerning the genetic predisposition of dissociative experiences is, however, mixed, as another study (Waller \& Ross, 1997) reports no genetic influence, while a recent study in children and adolescents does lend support to the notion of a genetic predisposition of dissociation (Becker-Blease et al., 2004).

Thus, the high prevalence and the possible heritable basis of dissociative phenomena make a direct causal link between dissociation and trauma less likely. It also underlines the importance of a different research perspective, namely one that focuses on the trait-like features of dissociation. One robust finding in this domain is that there exists a substantial overlap between dissociation and fantasy proneness (Merckelbach et al., 2000a; Rauschenberger \& Lynn, 1995; Waldo \& Merritt, 2000). Fantasy proneness refers to a deep and extensive involvement in fantasizing and daydreaming (Merckelbach \& Muris, 2001). While fantasy proneness is integral to healthy psychological functioning, an inability to control the cognitive processes implicated in imaginative involvement may be associated with psychopathology (Rauschenberger \& Lynn, 1995). Fantasy proneness has repeatedly been shown to be related to dissociation in both clinical (Merckelbach et al., 2005; Pekala et al., 1999-2000) and non-clinical samples (Merckelbach et al., 2000a; Merckelbach et al., 1999) with correlations ranging from. 41 (Pekalla et al., 1999-2000) to .55 (Merckelbach et al., 2000a).

Given the fact that fantasy proneness is associated with susceptibility to pseudo-memories, dissociation might also be related to a tendency to confabulate. Evidence for this comes from a study by Merckelbach et al. (2000a). Participants watched 40 slides, with 20 depicting photographs of common objects or situations and the other 20 being a short paragraph describing a scene or situation. Following this, participants were given a surprise recognition test in which they had to identify slides they had seen earlier in a series of old and new slides. They also had to indicate whether they had seen the "old" slide as a photograph or a paragraph. "Two types of errors may occur during this task. Participants may erroneously confuse a picture with a text or vice versa. This would be a reality monitoring error. Participants might also claim that they recognize a slide, which was not presented earlier. This would be a pseudo-memory, i.e., a commission error. Dissociative experiences were found to be related to commission errors, but not to reality monitoring errors.

In a more recent study, Candel, Merckelbach, and Kuijpers (2003) examined memory commission errors in a sample of undergraduate students scoring either high ( $n$ $=19)$ or low $(n=19)$ on the DES. The authors presented an emotional narrative to the participants, who then had to complete a free-recall task. Dissociation was associated with commission errors, but not with proportion correct recall, with high dissociators committing significantly more commission errors than low dissociators. In contrast to other studies (Merckelbach et al., 2000a), the link between dissociation and commission errors was not mediated by fantasy proneness. This, however, might have been due to the limited sample size in the Candel et al. (2003) study.

Our study attempted to replicate the Candel et al. (2003) findings, but also tried to extend our understanding as to why commission errors are associated with heightened 
dissociation. One possibility is that this association has to do with high dissociators suffering from limited working memory capacity. A limited working memory capacity might hamper encoding, leading to gaps in memory (Brewin \& Saunders, 2001), which are later filled in by participants, thereby leading to confabulations. Thus, the first issue examined in the current study is whether high dissociators' commission errors are related to working memory problems.

A second issue addressed by our study has to do with the deferisive function that is often ascribed to dissociative experiences (Gershumy \& Thayer, 1999). If this idea is correct, one would hypothesize that high dissociators show fewer hits on a mernory twsk for emotional material (i.e., one would expect more omission errors), as one of the core features of dissociation is psychogenic amnesia (American Psychiatric Association, 1994). During exposure to the emotional provocative material, one would also expect them to exhibit attenuated skin conductance responses as compared to low dissociators (e.g., Simeon, 2004). That is, one would predict that high dissociators are able to withdraw themselves psychologically from emotional events. Alternatively, if dissociative experiences and fantasy proneness describe a common domain, high dissociators" skin conductance responses should be more pronounced as compared to low dissociators due to their more pronounced imaginative involvement in the emotional material (Candel \& Merckelbach, 2003).

\section{Method}

\section{Participants}

Participants were 62 undergraduate students enrolled at the Maastricht University. Forty-five of them were women. Their mean age was 19.03 years $(S D=1.56$; range: 17 to 26 years). Participants were compensated through course credit points and gave written consent prior to taking part. The study was approved by the local ethical committee.

\section{Psychometric instruments}

Dissociative Experiences Scale (DES; Cronbach's $a=92$ ). The DES (Bernstein \& Putnam, 1986) is a self-report scale asking respondents to indicate on $100 \mathrm{~mm}$ visualanalogue scales to what extent they experience 28 dissociative phenomena in daily life. Examples of such phenomena include feelings of depersonalization, derealization, and psychogenic amnesia. The DES has high internal consistency and test-retest correlations ranging from.74 to .84. In a meta-analytic study, Van Uzendoorn and Schuengel (1996) provide evidence for the sound psychometric properties of the DES.

\section{Creative Experiences Questionnaite (CEQ. Cronbach's a $=.70$ ). The CEQ} (Merckelbach, Horselenberg, \& Muris, 2001) is a 25-item self-report measure of fantasy proneness sealed in the true/false form. It requires respondents to specify whether they exhibit various characteristics of fantasy proneness (e.g., "As a child, I thought that the dolls, teddy bears, and stuffed animals that I played with were living creatures." and "When I think of something cold, I actually get cold."). A total score is obtained by summing all yes answers. Merckelbach et al. (2001) reported adequate test-retest stability, internal consistency, and concurrent validity for this instrument. 
Digit Span forward and backward. The digit span forward is a standard measure of working memory capacity (Lezak, Howieson, \& Loring, 2004). A sequence of digits ranging from 1 to 9 of increasing length is presented to the participant who attempts to repeat it immediately. The number of digits in the longest sequence that has been repeated correctly is taken as the person's score. When the participant fails to recall two sequences of the same length the test is terminated. Digit span backward is similar to the digit span forward, except that the participant has to recall the digits in reversed order.

\section{Psychophysiological measures}

In the present study, one 3-min episode of skin conductance was sampled at $40 \mathrm{~Hz}$. While we recognize the limitations of only one physiological measure, we chose to measure skin conductance responses for the following two reasons. Firstly, skin conductance is a reliable indicator of sympathetic nervous system activity that can be measured unobtrusively (Kring \& Gordon, 1998). Secondly, there exists an extensive literature indicating that skin conductance is proportional to the emotionality of stimuli (Fowles, 1980). Skin conductance was measured with two silver/silver chloride $(\mathrm{Ag} / \mathrm{AgCl})$ electrodes filled with isotonic electrode gel and placed on the thenar and hypothenar eminences of the palm of the non-dominant hand. Before placement of the electrodes, participants rinsed their hands with distilled water. Respiration rate was measured with a strain gauge around the participant's waist. Respiration was recorded as control variable so as to make sure that skin conductance was related to the film fragment and not to respiratory irregularities. A personal computer was used for stimulus presentation, while a second personal computer running Psylab 7 (Dow, 1997) was used for on-line storage of SCR and respiration data. Stimuli were presented on a 19-inch color monitor. The timing of the video fragment and the skin conductance measure were synchronized.

\section{Stimulus material}

A highly emotional video fragment from the Hollywood film American History X (Kaye, 1998) was used. The fragment lasted approximately $2 \mathrm{~min}$ and depicted a neoNazi attacking a group of three African-Americans who try to steal his car. First, he shoots at them, ultimately forcing one of them to put his teeth on the sidewalk in order to step on his head. We selected this particular video fragment because previous work in our lab (Smeets, Candel, \& Merckelbach, 2004) made clear that participants evaluated it as emotionally provocative and that the video fragment led to an increase in both anxiety and tension. In more general terms, research (e.g., Jansen \& Frijda, 1994) shows that video clips may elicit strong emotional arousal in participants.

\section{Procedure}

Participants were tested individually in our lab with a female experimenter present. First, the DES and the CEQ were administered. Next, participants completed the digit span forward and backward. Then, participants were connected to the psychophysiological recording equipment and given $5 \mathrm{~min}$ to accommodate. Following this, they were instructed to watch the video fragment. At the end of it, the experimenter asked them to write down everything they could remember from the fragment. Participants were given sufficient time to complete this task. When the participants had completed this task, they were thanked for their participation and fully debriefed. 


\section{Statistical analyses}

Statistical analyses were performed using SPSS software. Cronbach's $\alpha$ 's were used to estimate the DES'S and CEV"S internal consistency.

Two independent raters, who were blind as to of the DES and CEQ scores of participants, evaluated free-recall performance (hits) using a set of 31 predefined criteria (Smeets et $\mathrm{al}, 2004$ ). The same ratets identified commission errors in participants" freerecall protocols. Commission errors were defined as details mentioned by the participant, which were clearly not present in the video fragment (e.g., "The AfricanAmerican man shoots back at the Neo-Nazi.'). Both hits and commission errors were averaged across raters.

Participants' skin conductance response (SCR) to exposure to the video fragment was measured in the $4 \mathrm{sec}$ window after the most emotional moment of the video clip. This moment lasted about one sec and depicted a neo-Nazi kicking on an African-American's head who is lying with his teeth on the sidewalk (i.e., Californian curve kick). We selected this event because free-recall transcripts collected during earlier experiments in our lab showed that it is experienced as an emotionally provocative fragment (see Smeets et al., 2004). SCR was defined as any deflection relative to baseline (measured during the 8 sec's preceding the onset of the film fragment; Lundgren, Berggren, \& Carlsson, 2004) exceeding 0.05 micro-siemens. To normalize the distribution, SCR data were square-root transformed.

To explore the possibility that high dissociators exhibited a pattern in which heiglitened skin conductance reactivity is followed by a steep decrease in reactivity (i.e., reflecting defensive processes; Friedman, 2000), we examined the time-course of skin conductance during an $8 \mathrm{sec}$ window following the maximum skin conductance response described above and calculated the decrement in skin conductance during this period relative to the maximum SCR.

Pearson product-moment correlations were calculated between the DES and all dependent measures. The mediational effect of fantasy proneness was investigated using the approach outlined in Baron and Kenney (1986).

\section{Results}

\section{Psychometric instruments}

Individual differences measures. Mean DES and CEQ scores were $17.86(S D=10.46)$ and $7.22(S D=3.73)$, respectively. These scores correspond with values that previous studies reported for student samples (Merckelbach et al., 2002). Table 2.1 presents Pearson product-moment correlations between DES, CEQ, and performance on the digit span task. As can be seen, there was a significant correlation between DES and CEQ scores. Thus, participants reporting more dissociative experiences exhibited stronger tendencies to fantasize.

Digit Span. Digit span forwand and backward were 6.18 $(S D=1.11)$ and 4.65 $(S D=$ 1.10), respectively. Correlations between digit span forward, backward, and dissociative experiences remained non-significant. 


\section{Recall performance}

Free-recall. Inter-rater reliability for hits and commissions were .85 and .82 , respectively. Participants recalled on average $36 \%(S D=8 \%)$ of the critical details, while making on average 0.37 ( $S D=0.58$ ) commission errors (in absolute numbers). Pearson product-moment correlations showed that both individual differences in dissociative experiences and fantasy proneness were positively related to commission errors, while not affecting hits (see table 2.1). It is worthy of note that previous research (Smeets et al., 2004) in our lab indicates that recall or emotional impact is unaffected by whether participants are familiar with the video fragment.

Table 2.1. Pearson product-moment correlations between dissociative experiences (DES), fantasy proneness (CEQ), Digit Span, free-recall performance, and commission errors for an undergraduate sample $(n=62)$. Only relevant correlations are shown.

Individual Difference Measures

DES CEQ

Individual

Difference

Measures

Digit Span

Free-recall
DES

CEQ

forward

Backward

proportion hits

commission errors

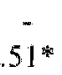

$-.11$

.15

$-.07$

$.34^{*}$
$.51^{*}$

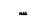

.10

.11

., 06

$.36 *$

* Correlation is significant at the .01 level (2-tailed).

One might wonder, whether the finding that dissociation and fantasy proneness are related to the number of commission errors in memory, is confounded by the self-paced nature of our free-recall task. The length of a written report might exert a strong influence on the reported content. That is why we calculated Pearson correlations between the number of words in the written report and the number of hits and commission errors. As expected, the number of hits was positively related to the number of words $(r=.64, p<.01)$, however, more interestingly, commission errors were unrelated to the number of words $(r=-.03, p>.05)$. Therefore, our finding that heightened levels of dissociation and fantasy proneness are related to commission errors in memory is not dependent on the length of the report participants give.

Modeling fantasy proneness as mediator during free-recall. The mediational effect of fantasy proneness on the relationship between dissociation and commission errors during the free-recall task was examined following the approach recommended by Baron and Kenny (1986). Firstly, we tested the unmediated model, consisting of the link between dissociation and commission errors in memory without controlling for fantasy proneness. Dissociative experiences, as measured with the DES, were found to predict commission errors during the free-recall task, $B=0.02, \beta=0.34, t(61)=2.78, p<.01$. 
Next, the mediated model was tested. This model consisted of dissociative tendencies, fantasy proneness, and commission errors during the free-recall task. Dissociative tendencies predicted fantasy proneness, $B=0.18, \beta=0.51, r(61)=4.60, p$ $<.01$, whereas a trend emerged with fantasy proneness predicting a tendency to commit memory commission errors, $B=0.04, \beta=0.25, t(61)=21.14, p=.07$. In addition, the effect of dissociation on memory commission errors decreased and was no longer significant, $B=0.01, \beta=0.21, f(61)=1.51, p=.14$. However, as it is not sufficient to show that the link between the independent variable and dependent becomes nonsignificant when the mediator is added to the model (Frazier, Tix, \& Barron, 2004), we calculated Sobel's (1982) test. This test indicates whether the indirect influence of the independent variable via the mediator is different from zero. Only a trend emerged, $z=$ $1.69, p=.09$, with $38 \%$ of the relation between dissociation and commission errors in free-recall being mediated by fantasy proneness.

\section{Skin conductance}

Dissociative experiences were positively correlated with SCR magnitude: $r=.34, p=$ .01 , whereas correlations between dissociative experiences and skin conductance baseline lewel remained non-significant $(r=-15, p>.10)$. Fantasy proneness was not related to $\mathrm{SCR}$ magnitude $(r=.18, p>.10)$.

Dissociation was unrelated to the dlecrement of skin conductance following the maximum SCR $(r=.03, p>.10)$. While there is no reason to expect that the $4 \mathrm{sec}$ window described above should differentially affect high and low dissociators, we excluded this possibility by calculating the maximum skin conductance response during the whole video fragment. This approach did not alter the correlation between dissociation and the skin conductance response $(r=.34, p<.01)$.

\section{Discussion}

The most important findings of the current study can be catallogued as follows. To begin with, heightened dissociation was related to commission errors during the free-recall task. Dissociation was, however, completely unrelated to the proportion of hits (i.e., accurate information elements). Secondly, in contrast to Candel et al. (2003), a mediator analysis revealed a trend with the link between commission errors and dissociation being partially mediated by fantasy proneness during free-recall. Thirdly, our findings cannot be explained by individual differences in working memory capacity, as all correlations with dissociation and working memory remained non-significant. Fourthly, dissociative tendencies were accompanied by a higher skin conductance response to the emotional video.

Our finding that high dissociators do not perform worse on an explicit memory task is in line with Sandberg, Lynn, and Matorin (2001), who reported that high dissociators do not exhibit degraded recall of danger cues from a video fragment. Our results are also in line with those of Brewin and Saunders (2001), who found that artificially inducing dissociation by means of a dual task during exposure to a video lragment does not lead to deterioration of recall performance. However, while these two previous studies clearly show that dissociation is unrelated to recall performance in terms of hits, both failed to look at commission errors. So far, only Candel et al. (2003) looked at whether dissociation is linked to the fabrication of details that were not present in the stimulus material (i.e., commission errors). In line with the 
aforementioned studies, these authors noted that the tendency to dissociate was unrelated to free-recall performance in terms of hits. Yet, Candel et al. (2003) did find evidence that dissociation was linked to commission errors in free-recall. They then explored whether the relationship between dissociative experiences and commission errors might be modulated by fantasy proneness. Candel et a1. (2003) found no evidence for such a mediating role of fantasy proneness. Replicating the results of Candel et al., our study showed that dissociation was linked to commission errors. While in the present clinical literature dissociation is rarely linked to confabulatory tendencies (but see Candel et al, 2003; Hyman \& Billings, 1998), the present finding reminds us of the historical roots of the concept of dissociation, which lie in the syndrome of hysteria (Kihlstrom, 1994). Interestingly, hysterics were renowned for their tendency to verbally exaggerate and for being fantasy ridden (e.g., Chodoff, 1974). In line with this assertion and unlike the Candel et al. study, we found some indications that fantasy proneness might partially mediate the dissociation-memory commission link. That Candel et al. were unable to detect this mediational role of fantasy proneness may have to do with two features of their study. One is that their sample was relatively small and consisted of students selected for their low and high DES scores. Another feature that might have obscured the role of fantasy proneness is the type of stimulus material employed by Candel et al. (2003). These authors exposed their participants to an emotional narrative that was read out loud. By presenting material only in the auditory modality, participants might have had the opportunity to fully concentrate on the material, which in turn, might have limited the room for commission errors. By relying on a highly emotional video clip, our stimulus material was more ecologically valid and may have allowed for a stronger influence of fantasy proneness on commission errors in memory. However, while this finding offers an explanation for high dissociators" susceptibility to pseudo-memories (Candel et al., 2003; Hyman \& Billings, 1998), it has to be interpreted with caution, as only a trend emerged regarding the mediating influence of fantasy proneness.

We found no evidence that working memory capacity is related to dissociation. This concurs with the fact that dissociation was not correlated with memory performance in terms of hits. Again, however, our failure to find a connection between dissociation and a traditional measure of short term memory has to be interpreted with caution. A clinical study by Cima, Merckelbach, Klein, Schellbach-Matties, and Kremer (2001) showed that cognitive differences between forensic patients high and low on dissociation might be connected to deficits in executive functioning. In addition, in one of our own studies (Giesbrecht, Merckelbach, Geraerts, \& Smeets, 2004), we found that dissociation was related to minor disruptions in executive functioning.

In the current study, higher dissociation scores were accompanied by a more pronounced skin conductance response to the critical scene of the emotional video fragment. Importantly, this pronounced skin conductance response was not followed by a dissociative or defensive process, which might serve to counter regulate this increased arousal. Our skin conductance findings are difficult to reconcile with the view that dissociative experiences constitute a defense mechanism that allows an indiwidual to psychologically withdraw from the impaot of the situation. Such a mechanism should reduce emotional engagement, thereby leading to lowered skin conductance responses. However, while skin conductance is a reliable and valid measure of emotionality (Fowles, 1980), future research relying on heart rate might further broaden our understanding of the supposedly defensive properties of dissociation. Heart rate is a valid measure of defensive responding. More specifically, defensive reactions are 
associated with heart rate acceleration (e.g., Verschuere, Crombez, Clercq, \& Emst, 2004). In addition, our study only measured dissociation as a trait, which means that our data does not provide any information about the current dissocialized state of our participants during the experimental condition. Therefore, future studies might also want to quantify state (i.e., peritraumatic) dissociation and its relation to both physiological responsiveness and commission errors in memory during emotional events in the laboratory.

If dissociation were to serve a defensive function, one would also predict that higher dissociation scores are accompanied by higher rates of forgetting. But, as said before, dissociation was not negatively related to hit rates (i.e., the inverse of forgetting). Other attempts to document a relationship between dissociation and defensive forgetting were also unsuccessful (e.g., Sandberg et al., 2001). One of the few studies that reported evidence that might be interpreted as support for the defensive function of dissociation was that by Koopman, Carrison, Butler, Sudhakar, Palmer, and Steiner (2004). These researchers found dissociative experiences to be associated with lowered mean heart rate in a sample of 41 delinquents. But, then the question arises whether this lowered mean heart rate reflects defensive blunting or a general deficit in arousall (see also Griffin, Resick, \& Mechanic, 1997). Clearly, the psychophysiology of dissociation is an under researched area and future studies addressing how tonic and phasic arousal relate to dissociation would be extremely welcome.

On the basis of our findings, we would argue that dissociative experiences, at least in our sample of undergraduate students, do probably not serve any defensive function. Watson $(2001 ; 2003 \mathrm{~b})$ pointed out that there is one alternative possibility that deserves serious consideration, which is that individual differences in sleeping behavior underlie variations in dissociative experiences (Giesbrecht \& Merckelbach, 2004; Watson, 2001, 2003b). The idea here is that high dissociators might more readily pass from normal waking into dream-like states, resulting in dissociative episodes and fantasy proneness. This idea is based on correlational studies showing robust associations between dissociation (as measured by the DES) and sleep experiences (as indexed with the lowa Sleep Experiences Survey; ISES; Watson, 2001, 2003b) ranging from 37 (Giesbrecht \& Merckelbach, 2004) to .54 (Watson, 2001). Positive response tendencies can be ruled out as a primary source of the connection between disturbed sleep pattens and dissociation (Giesbrecht \& Merckelbach, 2004). This connection does, however, not necessarily imply that tramatic experiences have no role in the development of dissociative symptoms. For example, Harvey, Jones, and Schmidt (2003) consider sleeping disruptions a typical reaction to traumatic events. Other factors, such as genetical predisposition (e.g., Lang et al., 1998), may further contribute to the sleep-dissociation link. Again, studies on the psychophysiology of dissociative experiences could shed more light on this issue. Thus, it would be interesting to measure background EEG in people high or low on dissociation. If high dissociators would exhibit typical sleep features in their waking EEG, this would constitute compelling evidence for a sleep-dissociation link (Giesbrecht \& Menckelbach, 2004; Watson, 2001).

To conclude, our findings are difficult to reconcile with the idea that dissociative experiences are manifestations of a defense mechanism, at least in our sample of undergradumte students. Plainly, new approaches to the study of dissociative experiences are needed. One promising avenue is the link between dissociative experiences and sleep distuptions. Studying differences in information processing and executive functioning between high and low dissociators might also broaden our insight. 
However, in contrast to previous studies (e.g., Sandberg et al., 2001), researchers should not limit their variables of interest to measures of hits, but should also take memory commissions into account. A combination of these approaches might contribute to a better understanding of the etiology of dissociative experiences. 


\section{Chapter 3: Trait dissociation and stress induced cortisol responses}

\section{Abstract}

The alleged defensive properties of trait dissociation, as measured by the Dissociative Experiences Scale, were studied in two samples of undergraduate students $(n=58$ and 67). Acute stress was induced by means of the Trier Social Stress Test. Subjective and physiological stress (i.e., cortisol) responses were measured. Dissociation was unrelated to cortisol responses, but positively related to subjective stress experiences, as indicated by the Tension-Anxiety subscale of the Profile of Mood States. This finding is in contrast with the notion that dissociative experiences constitute the manifestation of a defense niechanism.

* Giesbrecht, T., Smeets, T., Merckelbach, H., \& Jelicic, M. (submitted). Trait dissociation and stress induced cortisol responses. 


\section{Introduction}

More than a century ago, the French psychiatrist Pierre Janet (1889) drew attention to the phenomenon of psychological dissociation. In his book $L$ 'automatisme psychologicue, he conceptualized dissociation as a defensive response to psychological trauma. By this view, menories of nomal experiences are automatically integrated into appropriate schemas. Highly aversive experiences, however, cannot be readily fitted into existing schemas and are therefore split off from conscious awareness. Janet termed these unintegrated experiences subconscious fixed ideas. He believed that they are not readily accessible, buit nevertheless influence perception and behavior, while not fulfilling any function. Janet also assumed that in traumatized individuals, dissociating aversive experiences from consciousness often becomes a habitual way to cope with stress. He referred to this as "emotional constriction" (see van der Kolk \& van der Hart, 1989).

Janet"s theory of dissociation was nearly forgotten, but reemerged as a result of increased scientific interest in the devastating influence of overwhelming psychological experiences, such as was the case in Vietnam veterans or surviwors of sexual abuse. This renewed interest in dissociation led to the inclusion of dissociative disorders in the third edition of the Diagnostic and Statistical Manual of Mental Disorders (DSM JII; American Psychiatric Association, 1980). In this way, dissociation regained its status as an important psychiatric concept. Following Janet's tradition, recent clinical literature emphasizes the defensive properties of dissociation (Classen et al., 1993; Gershuny \& Thayer, 1999; Sanders \& Giolas, 1991; Zlotnick et al., 1996).

Ample evidence has been gathered, relating dissociative experiences to retrospective reports of a variety of traumatic experiences, such as childhood sexual and physical abuse (Brumer, Parzer, Schuld, \& Resch, 2000; Nash et al., 1993; Zlotnick et al., 1996). While these studies greatly contributed to our understanding of dissociative symptoms astonishingly little is know about high dissociators' responses to acute stress situations (but see Koopman et al., 2004; Sierra et al., 2002; Williams et al., 2003). This is surprising for two reasons. To begin with, Janet (in van der Kolk \& van der Hart, 1989) formulated explicit predictions about how individuals with habitually high levels of dissociative experiences would react under stressful situations. Relative to individuals with low dissociative levels, high dissociative individuals should, according to Janet, exhibit emotional constriction, manifested in lower psychophysiological reactions and less subjective stress experiences during emotionally provocative situations. Secondly, acute stress can be reliably induced in the laboratory and readily quantified using salivary cortisol levels (Dickerson \& Kemeny, 2004) and validated self-report measures of subjective stress experiences (e.g., Profile of Mood States; McNair, Lorr, \& Droppleman, 1992).

To examine the alleged defensive properties of trait dissociation, we measured subjective stress experiences and physiological stress (i.e., cortisol responses) in undergraduate students who were exposed to the Trier Social Stress Test (TSST; Kirschbaum, Pirke, \& Hellhammer, 1993). This task allows the induction of acute stress in the laboratory. In Jine with Janet (1889) and others (Classen et al., 1993; Gershuny \& Thayer, 1999), we expected higher levels of trait dissociation to go along with attenuated stress responses. 


\section{Study 1}

\section{Method}

\section{Participants}

Participants were 58 undergraduate students enrolled at Maastricht University.

Twenty-nine of them were women. Their mean age was 19.93 years $(S D=3.33$; range:

17 to 41 years). Participants gave written consent prior to taking part and were compensated through a small amount of money. Participants were asked to refrain from eating, drinking, and smoking for at least $1 \mathrm{~h}$ prior to the beginning of the testing session. The study was approved by the standing ethical committee of the Faculty of Psychology, Maastricht University.

\section{Psychometric instruments}

Dissociative Experiences Scale (DES; Bernstein \& Putmam, 1986, Cronbach's alpha = .92). The DES is a self-report scale asking respondents to indicate on $100 \mathrm{~mm}$ visual analogue scales to what extent they experience 28 dissociative phenomena in daily life. Examples of such phenomena include feelings of depersonalization, derealization, and psychogenic amnesia. In a meta-analytic study, Van IIJzendoorn and Schuengel (1996) provide evidence for the sound psychometric properties of the DES.

Profile of Mood States (POMS; McNair et al., 1992). The POMS is a self-report measure that is widely used to quantify typical and persistent mood reactions to current life situations. In the present study, subjective stress experiences were assessed using its Anger-Hostility and Tension-Anxiety subscales. Participants indicate to what extent they agree with adjectives describing their current mood or feelings on 5-point scales (anchors: $0=$ not at all, 4 = extremely). Adjectives include "annoyed", "angry", and "grumpy" for the subscale Anger-Hostility and "nervous", "tensed", and "panicky" for the Tension-Anxiety subscale. Total scores for the two subscales were calculated by adding up all item scores. The POMS has excellent psychometric properties (Lezak, 2004; Wald \& Mellenberg, 1990).

\section{Trier Social Stress Test}

The Trier Social Stress Test (TSST; Kirschbaum et al., 1993) combines both social and cognitive stress. It consists of three stages each lasting 5 min: a preparation period, a free-speech, and a mental arithmetic task. Thus, participants are first told to prepare a speech for a job interview, which they subsequently have to give. Following the speech, a mental arithmetic task is administered, which consists of counting backwards from 1022 in steps of 13. Every time the participant makes an error, she/he has to start from the beginning. Both the speech and the mental arithmetic task are videotaped and observed by the experimenter. The participant has to remain standing during these two latter stages. The TSST is a reliable method to induce psychological stress in the laboratory (see Kirschbaum \& Hellhammer, 1994) and was found to provoke the most pronounced physiological (cortisol) reaction as compared to other stress induction procedures (Dickerson \& Kemeny, 2004). 


\section{Procedure}

Participants were tested individually in our lab with the experimenter present. All experimental sessions took place in the afternoon (between 14.00 and $17.00 \mathrm{~h}$ ) in order to reduce variability in the cortisol response due to fluctuations of cortisol levels over the day (Kirschbaum \& Hellhammer, 1994). Participants first completed the DES. Next, pre-manipulation cortisol levels were sampled and participants completed the first POMS. Then, participants were exposed to the Trier Social Stress Test (TSST; Kirschbaum et al., 1993). Immediately after the TSST, a second cortisol sample was obtained and the POMS was re-administered. A third and fourth cortisol sample were taken, 20 and 40 min after the TSST, respectively.

\section{Saliva sampling and free cortisol analysis}

Cortisol data were obtained using Salivettes (Sarstedt, Nümbrecht, Germany). The uncentrifuged saliva samplles were stored at $-40^{\circ}$ Celsius immediately upon collection. Salivary free cortisol levels were determined in duplicate by direct radioimmunoassay, including a competition reaction between 125iodohistamine-cortisol and anti-cortisol serum made against the 3-CMO-BSA conjugate. Via a conventional 'second antibody" method, separation of free and antibody-bound 125 iodohistamine 9 cortisol was performed after overnight incubation at $4^{\circ}$ Celsius of $100 \mu \mathrm{l}$ of saliva. In order to reduce sources of variability, all 4 samples from an individual were analyzed in the same assay. Mean intra- and inter-assay coefficients of variation ranged from $4.3 \%$ to $8.3 \%$, respectively.

\section{Results}

Subjective stress experiences. The mean DES score was $16.61(S D=9.76)$. These scores correspond to values that previous studies reported for student samples (Merckelbach et al., 2002). Mean score on the Anger-Hostility and the Tension-Anxiety POMS subscales, obtained prior to the TSST, were $1.03(S D=2.88)$ and $5.16(S D=$ $2.46)$, respectively, and increased to $2.50(S D=3.29)$ and $9.00(S D=2.97)$, respectively after the TSST. Paired samples $t$-tests indicated that for both Anger-Hostility and Tension $\sim$ Anxiety post-manipulation scores were higher than pre-manipulation scores $[t(57)=3.24, p<.01$ and $t(57)=8.95, p<.01$, respectively]. This shows that our TSST manipulation succeeded in inducing psychological stress.

We computed difference scores reflecting pre- to post-manipulation changes on the two POMS subscales. Table 3.1 presents Pearson product-moment correlations between the DES and these pre- and post-manipulation change scores. Dissociation was neither related to the POMS Anger-Hostility $(r=.17$, ns.) nor to the Tension-Anxiety $(r$ $=-.06$, ns.) subscales prior to the manipulation (i.e., at baseline). However, dissociation correlated positively with changes on the Tension-Anxiety, but not with changes on the Anger-Hostility subscale.

Cortisol reaction. Mean baseline cortisol level was $4.00 \mathrm{nmol} / \mathrm{l}(S D=1.82)$. Salivary free cortisol levels throughout the study are displayed in Figure 3.1. Repeated measures ANOVA with the four cortisol levels as within-subject factor revealed a significant main effect of time of measurement $(F(3,55)=11.32, p<.01)$ indicating that our manipulation significantly increased cortisol levels in participants. 
Figure 3.1. Mean free salivary cortisol level (nmol/l) before stress manipulation ( 0 min), immediately after stress exposure (TSST; $15 \mathrm{~min}$ ), 20 min after stress exposure $(35 \mathrm{~min})$, and at the end of testing (55 min) for study $1(n=58)$ and study $2(n=67)$.

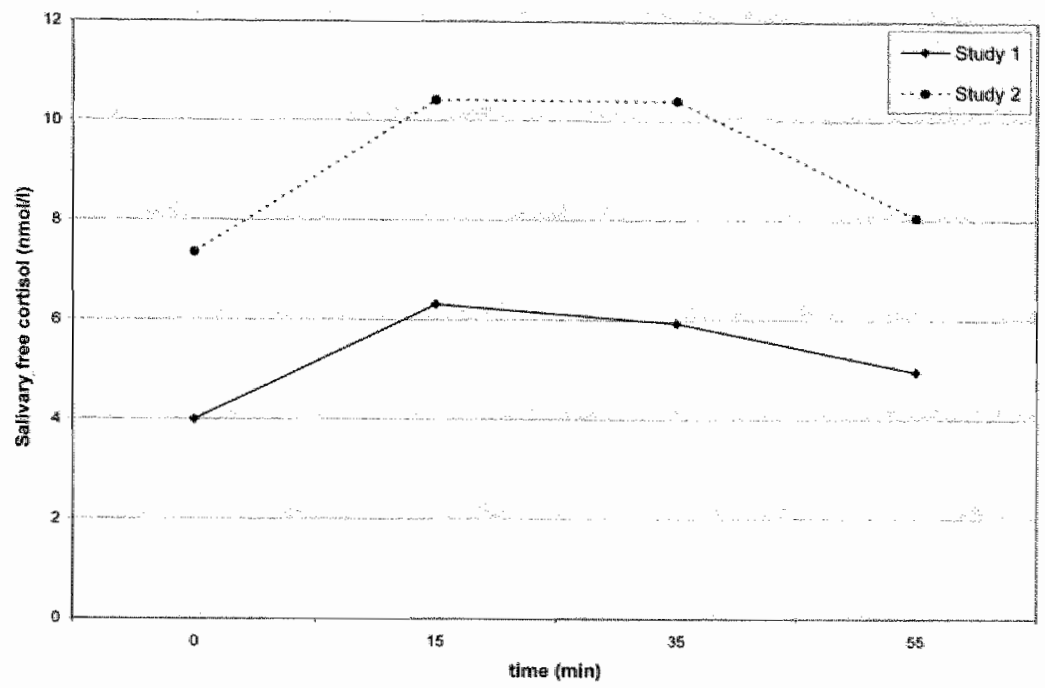

Following the recommendations of Pruessner, Kirschbaum, Meinlschmid, and Hellhammer (2003), we quantified the cortisol response in three ways. Firstly, we calculated the increase in cortisol level immediately after the manipulation (t15) with respect to the baseline (t0). In addition, we calculated the area under the curve with respect to ground $\left(A U C_{G}\right)$ and with respect to increase $\left(A U C_{1}\right)$. All correlations between DES and measures of the glucocorticoid stress response fell short of significance (see Table 3.1). In addition, baseline cortisol levels were unrelated to DES sores.

\section{Study 2}

Our finding that dissociation is not accompanied by an attenuated cortisol response during acute stress, i.e., the Trier Social Stress Test, is in sharp contrast with the alleged defensive function of dissociative experiences. However, due to our relatively small sample size, the aforementioned null findings have to be interpreted with caution and require replication. Therefore, we conducted a second study, which parallels study 1's procedure, while employing a modified version of the TSST.

\section{Method}

A sample of 67 ( 33 woman) undergraduate students enrolled at Maastricht University participated in our second study. Their mean age was 19.70 years $(S D=1.76$; range: 18 to 27 years). After completion of the DES, they all underwent our slightly modified version of the TSST. To make the TSST even more personally relevant, the job 
interview was replaced by a speech about their personality. Furthermore, the difficulty of the arithmetic task was increased by having participants solve a number of difficult subtractions. Our cortisol sampling procedure closely mirrored that employed in study 1. Subjective stress experiences were not measured during study 2.

\section{Results}

Cortisol reaction. Mean baseline cortisol levels were 7.36 nmol/I $(S D=5.90)$. Repeated measures ANOVA with the four cortisol levels as within-subject factor revealed a significant main effect of time of measurement $[F(3,64)=21.51, p<.01]$ indicating that our manipulation significantly increased cortisol levels (see figure 3.1 for salivary free cortisol levels).

Table 3.1. Pearson product-moment correlations between the Dissociative Experiences Scale (DES), the post-minus-pre manipulation changes on the Anger-Hostility and the Tension Anxiety Scale of the Profile of Mood States (POMS), cortisol baseline levels, increase from to (pre-manipulation) to 115 (post-manipulation), area under the curve with respect to ground $\left(A \cup C_{G}\right)$ and to increase $\left(A U C_{D}\right)$ for an undergraduate sample $(n$ $=58$ ). Only relevant correlations are shown.

\section{DES}

Study 1

Study 2

\begin{tabular}{lrcc}
\hline POMS & $\begin{array}{c}\text { Anger-Hostility } \\
\text { Tension-Anxiety }\end{array}$ & .20 & - \\
& Baseline & -.01 & - \\
& $\mathbf{A ( t 0 , t 1 5 )}$ & -.10 & -.20 \\
Cortisol & $\mathbf{A U C}_{\mathrm{G}}$ & -.15 & .10 \\
& $\mathbf{A U C}_{\mathbf{l}}$ & -.10 & -.14 \\
& & .18 \\
\hline
\end{tabular}

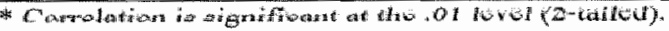

In study 2, cortisol responses were quantified as described earlier. In line with the findings from study 1 , all correlations between DES and measures of the glucocorticoid stress response fell short of significance (see Table 3.1).

\section{Discussion}

The most important findings of our two studies can be catalogued as follows. As indicated by elevated levells of both subjective experienced (in study 1) and physiological stress (in study 1 and 2), we successfully induced acute stress by means of the TSST. Dissociative experiences were, however, unrelated to baseline cortisol levels and cortisol responses to acute stress (i.e., cortisol responses) in our two samples of undergraduate students. On the other hand, individuals high on dissociation experienced a pronounced increcase in subjective stress as measured by the Tension-Anxiety subscale of the POMS. Clearly, this pattern does not support the idea, that dissociation 
has a defensive function. On the basis of this idea one would have predicted lowered levels of subjective and physiological stress responses.

Although we acknowledge the limitations of relying on only one phystological measure, the cortisol response is a widely used and valid measure of stress (Dickerson \& Kemeny, 2004). An interesting alternative parameter would be heart rate, as defensive reactions are associated with heart rate acceleration (e.g., Verschuere et al., 2004). Therefore, the relationship between dissociation and heart rate reactivity warrants further study. Psychophysiological studies conducted along these lines might broaden our understanding of the supposedly defensive properties of dissociation. Also a replication of our findings in clinical groups exhibiting severe dissociative symptoms would be highly informative, although ethical issues arise when using the TSST in such groups. In addition, the quantification of acute (i.e., peritraumatic) dissociative responses during acute stress and its relation to dissociation as a trait might be interesting.

To conclude, our finding that individuals with raised dissociative levels report more subjective stress and do not exhibit reduced physiological stress is difficult to reconcile with the defensive function commonly ascribed to dissociative experiences. 


\section{Part II: Dissociation, attentional control, and memory}

Basic cognitive processes involved in attention and menory most likely play an important role in dissociating explicit awareness of betrayal trauma.

- Jennifer Freyd, 1996 
" a 


\section{Chapter 4: Dissociative experiences in undergraduate students reflect lack of cognitive efficiency*}

\section{Abstract}

Undergraduate students scoring high $(n=22)$ or low $(n=24)$ on the Dissociative Experiences Scalle (DES; Bernstein \& Putnam, 1986) completed a selective attention and a dual attention version of the Stroop color-naming task. The tasks involved congruent, incongruent, baseline stimuli, neutral words, and trauma-related words that were combined with a free-recall task. Relative to participants low on the DES, undergraduates with high DES scores exhibited overall slowing of Stroop reaction times. The two groups did not differ in free recall. High DES participants' slowing of reaction times appeared to be related to individual differences in the susceptibility to cognitive failures. Our results suggest that dissociative experiences might reflect a general lack of attentional control.

"Chapter 4 is an adjusted version of the following article: Giesbrecht, T., Merckelbach, H., Sluis, A. F., \& Cima, M. (submitted). Critically evaluating the betrayal trauma theory of dissociative experiences - A negative finding. 


\section{Introduction}

The concept of dissociation is widely used in neuropsychology, neurology, and psychiatry. In the psychiatric domain, it is defined as "the lack of normal integration of thoughts , feelings, and experiences into the stream of consciousness and memory" (Bernstein \& Putnam, 1986, p. 727). This definition is in line with the Diagnostic and Statistical Manual of Mental Disorders (DSM IV; American Psychiatric Association, 1994), which considers symptoms such as derealization, depersonalization, and psychogenic amnesia as core features of dissociation. The commonly voiced view in psychiatric literature is that dissociative experiences constitute an automatic psychological defense mechanism against the impact of traumatic events. According to this view, trauma victims would display dissociative symptoms in order to withdraw themselves mentally from the impact of trauma. However, in the normal population mild dissociative experiences are highly prevalent, with $80 \%$ to $90 \%$ of the respondents indicating that they experience dissociative experiences at least some of the time (Gershuny \& Thayer, 1999).

The systematic study of dissociative experiences dates back to the end of the 19 th century when Pierre Janet (1889) was the first to introduce the term of psychological dissociation, a term that enjoys renewed interest during the last 20 years. Janet was also the first to relate dissociation to psychological trauma and he conceptualized dissociation as a defensive maneuver of trauma victims (Hacking, 1995). In this way, a habitual tendency to dissociate would promote psychopathology (van der Kolk \& van der Hart, 1989). Following this tradition, recent clinical literature emphasizes the causal relationship between trauma and dissociation (see, for a critical review, chapter 1; Kihlstrom, 2005; Merckelbach \& Muris, 2001). This relationship is widely accepted and is presented by many authors as uncontroversial (e.g., Gast et al., 2001: Gershuny \& Thayer, 1999).

The inclusion of dissociation in the previous version of the Diagnostic and Statistical Manual of Mental Disorder (DSM III; American Psychiatric Association, 1980) has led to the development of the Dissociative Experiences Scale (DES; Bernstein \&. Putnam, 1986). This instrument is now recognized as the standard instrument for measuring dissociative experiences and is extensively used to quantify dissociation in normal and clinical populations (Wright \& Loftus, 1999). The DES asks the respondent to indicate on $100-\mathrm{mm}$ visual analogue scales the frequency with which they experience 28 distinct dissociative symptoms (e.g., not recognizing friends or family members, finding oneself deeply involved in fantasy or daydreaming). Scores are then averaged across ifens to obtain a mean DES score.

A variety of studies found DES scores to be significantly related to retrospective self-reports of traumatic childhood experiences (Nash et al., 1993; Sandberg \& Lynn, 1992; Sanders \& Giolas, 1991). The interpretation of this finding is, however, extremely difficult given the fact that trauma was evaluated retrospectively and often with unvalidated, non-standardized questionnaires (Irvin, 1998; Sanders \& Giolas, 1991; Zlotnick et al., 1996).

A completely different research line focuses on the well-documented overlap between dissociation and fundamental personality traits, notably fantasy proneness and cognitive failures (Merckelbach et al., 2002). Cognitive failures refer to everyday lapses in perception, memory, and motor function and the frequency with which they occur can be measured with the Cognitive Failures Questionnaire (CFQ; Broadbent et al, 
1982). This self-report questionnaire asks the respondent to indicate how often they experience everyday lapses, like forgetting of names or getting distracted unwillingly on a 5-point scale (anchors: $0=$ never, $4=$ very often) and comprises of 25 items. A sample item is: "Do you fail to notice signposts on the road?" Scores on the individual items are surnmed to yield a total CFQ score. It is important to emphasize that self-reports of cognitive failures go hand in hand with poor performance on inhibitory tasks. This is underpinned by the study of Robertson, Manly, Andrade, Baddeley, and Yiend (1997). These authors showed that the performance on the Sustained Attention to Response Task (SART), a task that involves key presses to frequently presented non-targets, while requiring the participant to withhold motor responses to occasionai targets, significantly correlates with cognitive failures as indexed by the CFQ. Also of interest is that the correlations between DES and CFQ range from .43 (Merckelbach et al., 1999) to .51 (Merckelbach et al., 2002).

The overlap between dissociation and cognitive failures strongly suggests that it might be worthwhile to look at information processing characteristics of individuals scoring high on the DES. Unfortunately, few studies have followed that lead. A notable exception is the work by Freyd and co-workers (DePrince \& Freyd, 1999; Freyd et al., 1998) who used the Stroop task (Stroop, 1935) to investigate the attentional basis of dissociation. Freyd et al. (1998) administered a standard Stroop task involving color words, xxx's as baseline, and words from 4 neutral categories (i.e., kinship, animals, household and space) to undergraduates scoring high or low on the DES. Words were presented in colored ink on stimulus cards. Dissociation was found to be associated with color-naming latencies for incongruent color word (i.e, the standard Stroop effect), but not with color-naming latencies for other stimulus categories. Thus, high DES students displayed more Stroop interference than did low DES students, leading Freyd et al. (1998) to conclude that heightened levels dissociation are accompanied by disruptions in attentional control. In a follow-up study, DePrince and Freyd (1999) extended the standard Stroop task with emotional and neutral words. This task was combined with a surprise free-recall task that was administered after the Stroop procedure. On a second block of trials, a dual task version of the Stroop was employed such that participants had to respond as fast as possible while simultaneously encoding all stimuli. Again, this was followed by a free-recall task. In line with previous work, high DES students exhibited more interference during the traditional (i.e., selective attention) Stroop task. However, during the dual task version (i.e., divided attention) of the Stroop task, this interference disappeared. Furthermore, high DES students consistently reproduced fewer aversive words during free recall than did low DES students, a result that is only partially in line with findings reported by Holtgraves and Stockdalle (1997). These researchers found that higher scores on the DES were associated with poorer recall for both threatening and non-threatening words. On the basis of this pattern of findings, DePrince and Freyd (1999, p. 452) conclude that dissociative individuals have a preference for context, which involve multiple tasks. They state that "individuals who are highly dissociative have developed ways to cope in life that allow for their dissociation." This means that they "may learn to dual-task as a way of managing and controlling the flow of information."

While the studies of Freyd and co-workers represent an important and innovative contribution to the literature of dissociation, there are two points that can be raised. To begin with, in the Freyd et al. (1998) study, Stroop reaction times were measured in a rather crude way using a stopwatch and stimulus sheets. This method is prone to measurement errors threatening the reliability of effects. Secondly, Freyd and 
co-workers interpret their results in terms of disrupted attentional and memory processes that would be typical for trauma victims. While this interpretation might be correct, an alternative explanation is that these disruptions reflect a generalized susceptibility to cognitive failures, a trait that has strong heritability (Boomsma, 1998) and has little to do with traumatic experiences per se.

With these considerations in mind, the current study was conducted. Using a more sensitive measurement procedure, we sought tot replicate the attentional and memory disnptions that Freyd and colleagues found in their studies on high dissociative individuals. In addition, we wanted to know whether such disnuptions are related to individual differences in cognitive failures.

\section{Method}

\section{Participants}

During mass testing sessions, undergraduate students enrolled at Maastricht University were selected on the basis of their scores on the DES. Students with DES scores above 20 or below 10 were invited to participate in our study. Sixty-four participants agreed to take part in the experiment. However, 15 of them had to be excluded as they failed to fulfll the inclusion criterion (DES score $>20$ or $<10$ ) during the second time the DES was administered. Two further participants had to be excluded, one because of dyslexia and one due to color vision deficits. One other participant failed to follow instructions properly during the Stroop task and therefore was also excluded. The final sample involved 24 low DES individuals (mean DES $=5.67 ; S D=2.66$; range: $0.73-9.50,20$ women) and 22 high DES individuals (mean DES $=32.46 ; S D=10.52$; range: 20.64 $55.00,17$ women). It is worthy of note that the mean DES score for the two groups closely match values reported by Freyd and co-workers for their samples. Mean age of Jow and high DES groups were 20.5 years ( $S D=1.87$; range: $18-24$ years) and 20.3 $(S D=2.06$; range: $18-26$ years $)$, respectively. The low and high DES group did not differ with regard to age $[f(43)=.35, p=.73]$ or gender distribution $\left[\chi^{2}(\mathbb{1})=.84, p=\right.$ 361 . All participants had normal or corrected-to-normal vision, and were compensated through either course credit points or a small amount of money.

\section{Design}

The design followed a 2 (groups: low DES versus high DES) x 2 (tasks: selective attention Stroop task versus dual attention Stroop task) x 5 (stimuli: congruent color words, incongruent color words, baseline stimuli, neutral, and trauma-related words) set-up, with the frst factor being a between-subjects factor and the last two factors being within-subjects factors.

\section{Stimulus material}

The selective-attention Stroop task (Stroop, 1935) consisted of standard Stroop stimuli, which are congruent color words (e.g., blue printed in blue ink), incongruent collor words (e.g, blue printed in red ink) and baseline stimuli (e.g., xxx printed in blue ink). In addition, enotional Stroop stimuli, consisting of 12 trauma-related words (e.g.; "rape" printed in blue ink), were employed. A set of 12 farm words (e.g., "pig" printed in blue ink) that matched the trauma-related stimuli with respect to frequency (CELEX; Baayen, Piepenbrock, \& Gulikers, 1995) and word length served as baseline stimuli. During the divided-attention Stroop task, the same categories were used. However, this 
time a different set of 12 farm and 12 trauma-related words, equivalent to the aforementioned ones was employed.

\section{Apparatus}

Using ERTS software, Stroop stimuli were presented via an IBM-compatible personal computer and a 19-inch color monitor. Each stimulus was preceded by a $100 \mathrm{msec}$ blank. Following this, the word was displayed in lower case at the center of the screen in one of four colors (red, blue, yellow, or green). The word remained on the sereen until the subject had started to name its color aloud. When no verbal reaction occurred, the trial was aborted after $2000 \mathrm{msec}$ and treated as a missing value. Verbal reaction times were measured using a voice key (Lafayette, type 3050 AT) with $2.6 \mathrm{msec}$ accuracy (Experimental Timing Standards Laboratory, 2001). The experimenter marked trials on which errors occurred due to coughing or wrong color naming.

\section{Procedure}

Participants were tested individually in a sound-isolated room. First, DES (Bernstein \& Putnam, 1986; Cronbach's $\alpha=.96$ ) and CFQ (Broadbent et al., 1982; Cronbach's $\alpha=$ .89) were administered. Next, participants were instructed that stimulus words would presented in the middle of the screen in either of four different colors (blue, red, green, or yellow) and that their task was to ignore the meaning of the words, while identifying as fast and accurate as possible the color of the word. Participants were shown how to speak to the voice key located in front of them. In addition, they were told not to correct themselves, in case they committed an error and to proceed concentrated. Following this, participants engaged in practice sessions involving colored country names to ensure that they understood the task. Following the procedure of Freyd et al., participants completed a first block of selective attention Stroop trials followed by a surprise free-recall memory task. During free recall, participants were instructed to write down as many of the words they had seen during the preceding task as they could remember. Following a 2-min paced-break, particippants completed a divided-attention (i.e., dual) version of the Stroop task. This time, they were explicitly instructed to name the color of the word as fast as possible while remembering as many of the words as possible. Again, this was followed by a free-recall memory task. Both Stroop tasks consisted of 24 congruent, 24 incongruent, 24 baseline, 24 neutral, and 24 traumerelated stimuli presented in random order. Neural and trauma stimuli were 12 different stimulus words each, presented twice during the block. In order to prevent recency effects, both Stroop task ended with a set of 8 country words. Finally, the participants were completely debriefed and thanked for their participation.

\section{Results}

\section{Cognitive failures}

An independent samples t-test performed on CFQ scores showed that the high DES group did report a substantially higher rate of cognitive failures than the low DES group, means being $32.75(S D=7.07)$ and $48.54(S D=10.60), t(44)=-5.99, p<.001$. 


\section{Stroop reaction times}

Reaction times for trials with incorrect responses were excluded from the analysis. To normalize the reaction time distribution, data were $\log$ transformed. In addition, logtransformed reaction times falling more than $3 \mathrm{sd}$ s from the mean reaction time of the participant were deleted. Mean Stroop reaction times for different categories of stimuli during the two different Stroop tasks are presented in figure 4.1. The overall difference between the low and the high DES group attained significance $[t(43)=3.32, p=.04$, one-tailed]. This group difference disappeared when CFQ scores were entered as a covariate $[F(1.42)=1.80, p=.19]$. The 2 (high vs. low DES) $\times 2$ (Stroop version) $\times 5$ (stimulus category) Analysis of Variance (ANOVA) with repeated measurements on the last two factors further revealed main effects for Stroop version $[F(1,44)=70.19, p<$ $.001]$ and stimulus category $[F(1,41)=109.58, p<.001]$. Only the interaction between Stroop version and stimulus category attained significance $[F(1,41)=8.08, p<.001]$. This effect has to do with the fact that both trauma and farm word stimuli led to longer reaction time in the dual task condition. All other interactions failed to meet conventional levels of significance.

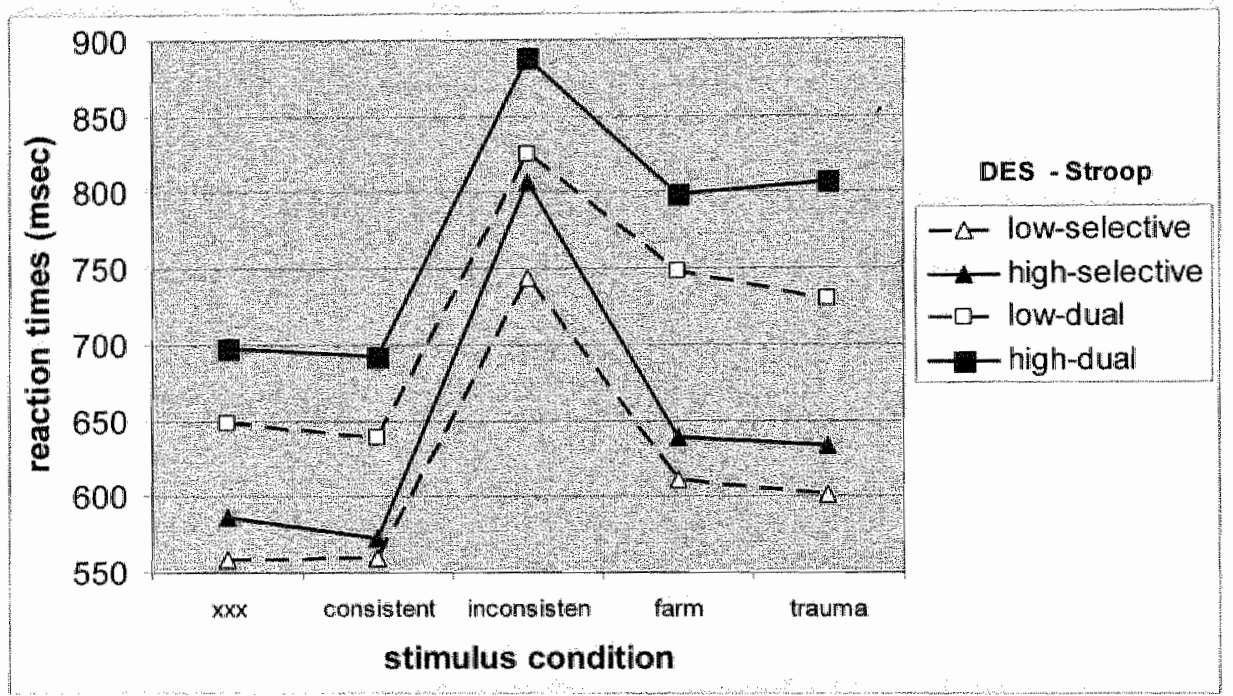

Figune 4. 1. Mean Stroop reaction times for high $(n=22)$ and low dissociative $(n=24)$ individuals as a function of attentional task (selective versus dual Stroop Color Naming Task) and stimulus condition.

\section{Free recall}

Table 4.1 shows the free-recall data. The two groups did not differ with regard to their overall free-recall performance $[t(43)=1.89, p=.18]$. A 2 (high vs. low DES) $\times 2$ (Stroop version) $\times 2$ (stimulus category) ANOVA with repeated measurements on the last two factors revealed main effects for Stroop version $[F(1,43)=142.63, p=.001]$ and stimulus category $[F(1,44)=13.37, p<.001]$. The main effect of Stroop version reflects, of course, the overall higher levels of free recall after the dual task version of the Stroop. The main effect of stimulus category was due to a larger proportion of 
neutral words that both group reproduced in comparison to their recall of trauma-related words. All other comparisons failed to acquire conventional levels of significance.

Table 4.1. Mean percentage of correctly recalled neutral and emotionally charged words by attentional task (selective and dual Stroop Color Naming Task) and high $(n=22)$ versus low DES $(n=24)$ group.

\begin{tabular}{cccccc} 
& \multicolumn{2}{c}{ Selective attention Stroop } & & \multicolumn{2}{c}{ Dual attention Stroop } \\
\cline { 2 - 3 } \cline { 5 - 6 } Group & Neutral & Trauma & & Neutral & Trauma \\
\hline Low DES & 21.74 & 13.77 & & 47.83 & 41.67 \\
High DES & 16.29 & 11.74 & & 44.70 & 40.91 \\
\hline
\end{tabular}

\section{Discussion}

In line with previous research (Merckelbach et al., 2002), higher scores on the DES were associated with substantially higher rates of self-reported cognitive failures. In addition, high dissociative individuals performed worse during all conditions of the selective as the dual attention version of the Stroop task. This means that, compared to low DES individuals, high DES individuals exhilbit an overall slowing of Stroop reaction times. This slowing appeared to be related to individual differences in cognitive failures, as the effect disappeared once cognitive failures were entered as covariate. The two groups did not differ with regard to their performance during the free-recall task.

Our findings stand in sharp contrast to those reported by Freyd et al. (1998) and DePrince and Freyd (1999). Recall that these authors found that relative to low DES individuals, high DES persons exhibit a selective slowing for conflicting color words, a difference that disappears once high DES persons perform under dual task conditions. These authors also reported that high DES individuals recall fewer trauma-related words than low DES individuals. Neither of these specific effects were reproduced in our study. This discrepancy might be due to the following reasons. Firstly, we included only individuals who showed reliable high or low DES scores, as indicated by the fact that they fulfilled the inclusion criterion twice. This strict inclusion strategy minimizes ertor variance in DES and ultimately prevents wrong group classification. That this is an important point is shown by the fact that in 15 out of 64 selected participants $(23.4 \%)$, DES scores were highly labile. The issue of DES stability and potential context effects that compromise this stability will be addressed in chapter 6 . Secondly, in contrast to Freyd et al. (1998), our study relied on a computerized version of the Stroop task, allowing a more precise measurement of color naming latencies. Admittedly, both the work of DePrince et al. (1999) and our study indicate that dissociative individuals exhibit a lack of attentional control. However, whereas the findings of Freyd and coworkers suggest that this attentional dysfunction is domain specific (i.e., depending on instruction and stimulus material), our work demonstrates that it is of a more generalized nature. In addition, Freyd and co-workers suggest that the cognitive dysfunctions extends to memory of emotionally laden material, whereas we found no evidence for this. In a way, these discrepancies remind us of discussions about $O C D$ symptoms, where some experts have argued that they reflect a general deficit, whereas others have conceptualized them as domain-specific (i.e., related to thoughts with an intrusional quality) phenomena (see, for an analysis of this discussion, Salkovskis, 
1999). As for dissociative symptoms, we are quite confident that they first and foremost reflect a subtle general deficit. The reason for this is the substantial difference between high and low dissociative individuals with regard to their self-reported cognitive failures (Merckelbach et al., 2002; Merckelbach et al., 1999).

Our findings demonstrate that dissociative experiences are related to (minor) attentional disruptions. The origins of these disruptions warrant further study. For example, it might be informative to look at differences in executive (i.e., frontal lobe) functioning between high and low dissociative indiwiduals. Note that there some indications that dissociation is related to subtle frontal lobe abnormalities. A more systematic study of these abnormalities might use different paradigms that are know to tap different aspects of executive functions, such as shifting, inhibition and updating (Myake, Friedman, Emerson, Witzki, \& Howerter, 2000). One related issue that comes to mind is the effect of poor attentional control on thought suppression. To the extent that successful thought suppression is highly dependent on effective attentional control, one would expect that dissociation is implicated in poor thought suppression (see chapter 6). However, so far this issue has not been fully addressed by researchers. Meanwhile, such research avenues might shed more light on the proximate mechanisms anderlying dissociative experiences.

Dozens of studies have focused on the relationship between dissociation and reports of childhood trauma (for a review, see Merckelbach \& Muris, 2001). While this focus on distal processes (i.e., childhood trauma) related to dissociation have undoubtly revealed highly relevant findings, it is now probably time to address the cognitive processes involved in dissociation. The field of anxiety disorders is a fine example of how such a cognitive analysis might contribute to a better understanding of the etiology and treatment of symptoms (e.g., Williams, Mathews, \& MacLeod, 1996). 


\section{Chapter 5: Dissociation in undergraduate students: Disruptions in executive functioning}

\section{Abstract}

The concept of dissociation refers to disruptions in attentional control. Attentional control is an executive function. Few studies have addressed the link between dissociation and executive functioning. Our study investigated this relationship in a sample of undergraduate students $(n=185)$, who completed the Dissociative Experiences Scale (DES; Bernstein \& Putnam, 1986) and the Random Number Generation Task ( $R N G$; Horne, Evans, \& Orne, 1982). We found that minor disruptions in executive functioning were related to a subclass of dissociative experiences, notably dissociative amnesia and the Dissociative Experiences Scale Taxon (DES-T; Waller et al., 1996). The two other subscale of the DES, measuring depersonalization and absorption, were, however, unrelated to executive functioning. Our findings suggest that a failure to inhibit dominant responses might contribute to the pathological memory manifestations of dissociation.

* Giesbrecht, T., Merckelbach, H., Geraerts, E., \& Smeets, E. (2004). Disruptions in executive functioning and dissociation in undergraduate students. Jownal of Nervous and Mental Disease, 192, 567-569. 


\section{Introduction}

According to Bernstein and Putnam (1986, p. 727), "dissociation is a lack of the normal integration of thought, feeling, and experiences into the stream of consciousness and memory." While some degree of dissociative symptoms are common in the general population, with $80 \%$ to $90 \%$ of the respondents indicating they experience such symptoms at least some of the time (Gershiuny \& Thayer, 1.999), an habitual tendency to dissociate is considered a defensive maneuver to withdraw from the impact of traumatic events. Accordingly, much research in this domain has focused on the relationship between trauma and dissociation.

Despite Bernstein and Putnam's (1986) inclusion of memory dysfunctions in their definition of dissociation, there is virtually no research on individual differences in basal information processing mechanisms associated with different levels of dissociation. A noteworthy exception is the work of Freyd et al. (1998) and DePrince and Freyd (1999). Their studies compare high and low dissociators with regard to performance on the Stroop task (Stroop, 1935), which is a measure of selective attention (see chapter 4 for a replication of their methodology). High dissociators exhibited greater Stroop interference, leading the authors to conclude that "dissociative tendencies were related to a basic cognitive operation and not dependent on the content of the material" (DePrince \& Freyd, 1999, p. 449). Their conclusion is underpinned by the fact that dissociation, as measured on the Dissociative Experiences Scale (DES; Bernstein \& Putnam, 1986), overlaps with susceptibility to everyday cognitive failures (e.g., failing to notice signposts on the road), as measured on the Cognitive Failures Questionnaire (CFQ; Broadbent et al., 1982), with correlations ranging from .43 (Merckelbach et al., 1999) to .5 I (Merckelbach et all, 2002). High scores on the CFQ have been linked to poor performance on the Sustained Attention to Response Task (SART; Robertson et al., 1997), a measure that is sensitive to attentional deficits.

A connection between dissociation and fundamental cognitive operations is also suggested by a study by Cima et al. (2001). These authors found in a sample of forensic inpatients that heightened levels of dissociative symptoms were accompanied by poor performance on tasks measuring executive (i.e., frontal) functions.

With these findings in mind, we conducted a study, which explored the possible links between aspects of dissociation and executive functions. To this end, we used the Random Number Generation Task. This measure has been extensively employed in clinical (Horne et al., 1982; Williams, Moss, Bradshaw, \& Rinehart, 2002) and non-clinical samples (Ginsburg \& Karpiuk, 1994). We hypothesized that differences in executive functioning might be related to certain dissociative symptoms as measured by the DES and its subscales. The DES comprises several subscales (Carlson et al., 1991 ). One of these - absorption - is more related to non-pathological manifestations of dissociation, whereas others (i.e., depersonalization/ derealization, amnesia, and DES-T) are thought to tap the malign aspects of dissociation (Waller et al., 1996). Thus, we tested the idea that poor executive functioning is related to the malign, but not the benign aspects of dissociation. 


\section{Method}

\section{Participants}

Participants were 185 undergraduate students (129 women) enrolled at the Maastricht University. Their mean age was 20.4 years ( $S D=2.36$; range: 17 to 30 years).

Participants were compensated through either course credit points or a small amount of money. The study was approved by the local ethical committee.

\section{Measures}

Dissociative Experiences Scale (DES; Cronbach"s $\alpha=93$ ). The DES (Bernstein \& Putnam, 1986) is a self-report scale asking respondents to indicate on $100 \mathrm{~mm}$ visual analogue scales to what extent they experience 28 dissociative phenomena in dally life. Examples of such phenomena include feelings of depersonalization, derealization, and psychogenic amnesia. A subset of 8 DES-items forms the Dissociative Experiences. Scale Taxon (DES-T; Cronbach's alpha $=.81$; Waller et al., 1996), which is thought to be especially sensitive to pathological dissociation. DES-T scores can be obtained by averaging across DES items 3, 5, 7, 8, 12, 13,22, and 27 (e.g., Eisen \& Carlson, 1998).

In addition to the DES-T, we calculated separate subscale scores. Following the three-factor solution proposed by Ross et al. (1995), Sanders and Green (1994), and Carlson et all (1991), we focused on three DES subscales. These were amnesia (Cronbach's $\alpha=.80$ ), absorption and imaginative involvement (Cronbach's $\alpha=.89$ ), and depersonalization and derealization (Cronbach's $\alpha=.85$ ).

Random Number Generation ( $R N G$ ). The RNG task requires the participant to generate random sequences of numbers ranging from 1 to 10 on the beep of the metronome (Myake et al, 2000). In the current study, participants were asked to cone up with 100 numbers. Various studies have employed this task to tap executive functioning (e.g., Horne et al., 1982; Williams et al., 2002). Three different parameters of deviations from randomness have been identified. Firstly, Repetition (e.g., 2,2) is the duplication of a number in succession and represents the inability to inhibit previous responses.

Secondlly, Series (e.g., 1, 2, 3) is related to deficits in schema inhibition. Thirdly, Poker represents the failure to use every possible alternative, before repeating any other digit and is indexed by the number of repetitions of the same digit within a sequence of tive items (e.g., 1, 5, 9, 1, 3). The poker score is linked to monitoring of previous responses (Williams et al., 2002). As all these paraneters are measures of deviations from randomness, higher values indicate less random responding and, therefore, relatively poor executive functioning.

\section{Procedure}

Participants were tested individually in our lab with an experimenter present. First, the Dissociative Experiences Scale (DES) was administered. Next, participants completed the Random Number Generation Task. Finally, the participants were thanked for their participation and completely debriefed. 


\section{Results}

Mean DES and DES-T scores were $16.91(S D=11.45)$ and $9.93(S D=10.40)$, respectively. Mean values for Repetition, Series, and Poker were 1.59 $(S D=2.99)$, $32.67(S D=7.44)$, and 4.71 $(S D=3.42)$, respectively. It is noteworthy that the mean DES values (Merckelbach et al., 2000a; Merckelbach et al., 2000b) and mean values for the RNG parameters (Ginsburg \& Karpiuk, 1994) correspond with values that previous studies reported for student samples, while DES-T values were slightly higher than usual (e.g., Watson, 2003a).

Petarson-product-moment correlations between total DES scores and all RNG parameters fell short of significance (see table 5.1). However, DES-T scores were significantly related to Repetition and Poker of the RNG task, but not to Series. Interestingly, the dissociative amnesia subscale of the DES followed the same correlational pattern. That is, this subscale correlated with Repetition and Poker, but not with Series (see table 5.1). Conversely, the Pearson correlations between the other DES subscales and RNG parameters remained non-significant.

Table 5.1. Pearson product-moment correlations between Dissociative Experiences Scale (DES), subscales of the DES, and parameters of the Random Number Generation Task (RNG) in an undergraduate sample $(n=185)$. Only relevant correlations are shown.

DES subscales

\begin{tabular}{|c|c|c|c|c|c|c|}
\hline & & \multirow[b]{2}{*}{ DES } & \multicolumn{3}{|c|}{ DES subscales } & \multirow[b]{2}{*}{ depersonalization } \\
\hline & & & DES-T & amnesia & absorption & \\
\hline & DES & - & & & & \\
\hline \multirow{4}{*}{ DES subscales } & DES-T & $.89 *$ & - & & & \\
\hline & amnesia & $.82 * *$ & $.73^{*}$ & - & & \\
\hline & absorption & $.94^{\text {*as }}$ & $.77^{* * *}$ & $.67^{*}$ & - & \\
\hline & Ination & .79 米将 & $.91 * *$ & $.54 * *$ & $.67 * 3$ & - \\
\hline \multirow{3}{*}{ RNG } & repetition & .13 & $.17^{*}$ & $.20 * *$ & .07 & .10 \\
\hline & series & .06 & .11 & .08 & .03 & .13 \\
\hline & polier & .12 & $.20 * *$ & $.23 *$ & .02 & .114 \\
\hline
\end{tabular}

* Correlation is significant at the .01 level (2-tailed).

* Correlation is significant at the 05 level (2-lailed).

\section{Discussion}

This study is the first systematic attempt to relate dissociative experiences, as measured by the DES and its subscales, to various parameters of executive functioning. In line with previous studies that focused on basal attentional mechanisms (DePrince \& Freyd, 1999; Freyd et al., 1998; chapter 4), frontal lobe tasks (Cima et al., 2001), or everyday cognitive lapses (Merckelbach et al., 1999), we found that minor disruptions in executive functioning were related to a subclass of dissociative experiences, notably dissociative amnesia and DES-T. The two other subscales of the DES, measuring depersonalization and absorption, were not related to executive functioning. 
Thus, our data suggest that there is a significant, albeit modest, relationship between failures to inhibit dominant responses (as indexed by Repetition and Poker), on the one hand, and the pathological memory manifestations of dissociation (amnesia, DES-T), on the other.

While the significant correlations that we found between the more pathological aspects of dissociation and executive functioning as indexed by RNG were not large, this may reflect our sample. After all, our study relied on undergraduates in whom variations in executive functioning will be relatively small. Our findings suggest that links between dissociation and executive function warrant further study.

This line of research might shed light on the proximal antecedents of dissociation. So far, most studies have emphasized distal antecedents, in particular traumatic childhood experiences (e.g. Gershuny \& Thayer, 1999). However, precisely how early aversive experiences might shape a habitual tendency to dissociate is unknown. Perhaps, then, such early aversive events might compromise frontal functioning, and in this way produce trait-like dissociative phenomena.

Our study indicates that it would be useful to systematically explore differences in executive functioning between high and low dissociators. Tasks known to tap the different aspects of executive functioning, such as updating, shifting, and inhibition (Myake et al., 2000) might further our insight into the cognitive processes involved in dissociation. In addition, the finding that the DES-T correlates with two of the three parameters of executive functioning suggests the value of extending our investigation to clinical samples with even more pronounced deficits in executive functioning. 


\section{Chapter 6: First you find them, then you don't- Correlations between thought suppression and dissociation and their context sensitivity}

\section{Abstract}

Various research (Muris \& Merckelbach, 1997; Spinhoven \& van der Does, 1999; van den Hout, Merckelbach, \& Pool, 1996) found that dissociative experiences, as measured with the Dissociative Experiences Scale (DES; Bernstein \& Putnam, 1986) are related to thought suppression, as measured with the White Bear Suppression Inventory (WBSI; Wegner \& Zanakos, 1994). This issue was further investigated in two samples of undergraduate students. Study I $(n=220)$ replicates and extends previous research in that it found a robust correlation between DES and WBSI, even when more pathological manifestations of dissociation (i.e., DES-T; Waller et al., 1996) were taken into account. Study $2(n=40)$ shows that more pathological features of dissociation are related to poor performance on a thought suppression task using highly emotional stimulus material. In addition, study 2 illustrates the susceptibility of the DES to context effects.

"Giesbrecht, T., Merckelbach, H., \& Smeets, E. (submitted). First you find them, then you don't - Thought suppression, dissociation, and context effects. 


\section{Introduction}

According to Bernstein and Putnam (1986, p. 727), "dissociation is a lack of the normal integration of thoughts, feelings, and experiences into the stream of consciousness and memory." However, the systematic study of dissociation dates back to the beginning of the $19^{\text {th }}$ century when Janet (1889) coined the term of dissociation and conceptualized it as a defensive maneuver of trauma victims (Hacking, 1995). During the last 20 years, the study of dissociation has enjoyed renewed interest and many authors followed Janet's tradition emphasizing traumatic experiences as the causal antecedent of dissociation (Brunner et al., 2000; Gershuny \& Thayer, 1999).

Another defense mechanism listed in the Diagnostic and Statistical Manual of Mental Disorders (DSM IV; American Psychiatric Association, 1994, p. 757) is suppression, which is defined as "avoiding thinking about disturbing problems, wishes, feelings, or experiences." Various studies found suppression, as measured with the White Bear Suppression Inventory (WBSI; Wegner \& Zanakos, 1994), and dissociation, as measured with the Dissociative Experiences Scale (DES; Bernstein \& Putnam, 1986), to be related to each other (Muris \& Merckelbach, 1997; Spinhoven \& van der Does, 1999; van den Hout et al., 1996). The theoretical importance of this lies in the suggestion that dissociation is not an automatic, unconscious defense mechanism, as is often assumed (Classen et al., 1993), but rather a deliberate effortful strategy. While this finding might provide new insights into possible mechanisms underlying dissociative experiences, two methodological shortcomings limit its value. Firstly, the measurement of thought suppression in these studies critically relied on a self-report scale (i.e., the WBSI). However, while there are some good reasons to believe that WBSI scores correspond with behavioral indices of thought suppression (Merckelbach, Muris, Nijman, \& de Jong, 1996), the possibility still exists that this self-report is sensitive to response biases in certain research contexts. However, a more substantial problem has to do with the issue of what the WBSI measures. Thus, one could argue that this instrument does not tap the disposition to suppress unwanted thoughts, but rather susceptibility to thought intrusions, which may originate from unsuccessful thought suppression attempts (Wegner, Schneider, Carter, \& White, 1987). On the other hand, such intrusions could stem from a lack of thought suppression attempts, which may be related to a breakdown of psychological defenses (Hoeping \& de Jong-Meyer, 2003).

The two studies to be described below sought to investigate the relationship between dissociation and thought suppression in two non-clinical samples. Firstly, we tried to replicate Muris and Merckelbach's (1997) and van den Hout et al. "s (1996) finding that dissociation and thought suppression are related to each other, while extending it to the more pathological facets of dissociation as measured with the DES-T (Waller et al., 1996). Secondly "we examined whether dissociation is related to suppressive capabilities, as measured during a behavioral task, i.e., a thought suppression experiment (see Wegner et al., 1987).

\section{Study 1: Method and results}

The Dissociative Experiences Scale (DES; Bemstein \& Putnam, 1986) and the White Bear Suppression Inventory (WBSI; Wegner \& Zanakos, 1994) were administered to 220 undergraduate students ( 150 women) enrolled at the Maastricht University. Their 
mean age was 19.69 years ( $S D=2.39$; range: 17 to 40 years). Participants were compensated through a small amount of money. The questionnaires were completed during a mass testing session at the beginning of the semester. The study was approved by the standing ethical committee of our faculty.

The DES (Cronbach's $\alpha=.90$ ) is a self-report scale asking respondents to indicate on $100 \mathrm{~mm}$ visual analogue scales to what extent they experience 28 dissociative phenomena in daily life. Examples of such phenomena include feelings of depersonalization, derealization, and psychogenic amnesia. A subset of 8 DES-items forms the Dissociative Experiences Scale Taxon (DES-T; Cronbach's alpha $=.74$; Waller et al., 1996), which is thought to be especially sensitive to pathological dissociation. Typical DES-T items are "Some people sometimes have the experience of feeling that other people, objects, and the world around them are not real" and "Some people sometimes find that they hear voices inside their head that tell them to do things or comment on things that they are doing.." DES-T total score can be obtained by averaging across DES items 3, 5, 7, 8, 12, 13, 22, and 27 (e.g., Eisen \& Carlson, 1998). In addition, Waller et al. (1996) recommended a way to derive Bayesian taxon membership probabilities from these 8 items (see Waller \& Ross, 1997). These probabilities reflect the chance that individuals belong to the pathological dissociative taxon. In the current study, we also calculated these taxon probabilities. ${ }^{3}$

The WBSI (Cronbach ${ }^{*} \alpha=.85$ ) is a 15-item self-report scale measuring people"s general tendency to suppress unwanted negative thoughts. The respondent is asked to indicate on 5 -point scales $(1=$ strongly disagree, $5=$ strongly agree) to what extent they agree with statements about their suppression of unwanted thoughts. Typical examples of the WBSI items are "I wish I could stop thinking of certain things." and "Sometimes I stay busy just to keep thoughts from intruding in my mind."

Mean DES, DES-T, and WBSI scores were $18.34(S D=10.53), 10.97(S D=$ $9.87)$, and $48.29(S D=9.90)$, respectively. These scores correspond with values that previous studies reported for student samples (Merckelbach \& Jelicic, 2004; Muris \& Merckelbach, 1997). Table 6.1 shows Pearson product-moment correlation between the DES, DES-T, DES-T taxon probability, and the WBSI. As can be seen, dissociative experiences as measured by the DES were related to the suppression of unwanted thoughts as measured by the WBSI. In addition, both average DES-T scores and DES-T taxon probabilities, which are thought to index the more pathological forms of dissociation (Waller et al., 1996), were related to thought suppression. The correlation between the DES-T taxon probability and thought suppression was, however, smaller than the two other correlations.

Thus, our findings show that the connection between dissociation and thought suppression is a robust one. However, the question arises whether dissociation is related to a behavioral index of suppression that is less susceptible to response bias. This issue was addressed by study 2 .

${ }^{3}$ We are well aware of the studies that cast doubt on the psychometric merits of the DES-T and especially its taxon membership probabilities (see page 140). However, these studies appeared after the data reported here were gathered and analyzed. 
Table 6.1. Pearson product-moment correlations between Dissociative Experiences Scale (DES), the Dissociative Experiences Scale Taxon scores (DES-T), Dissociative Experiences Scale Taxon probabilities (DES-T Taxon), and the White Bear Suppression Inventory (WBSI) in an undergraduate sample $(n=220)$.

\begin{tabular}{lcccc} 
& & & DES-T \\
& DES & DES-T & Taxon probability & WBSI \\
\hline DES & - & $.84^{*}$ & $.56^{*}$ & $.39^{*}$ \\
DES-T & - & $.77^{*}$ & $.35^{*}$ \\
DES-T Taxon probabillity & & - & $.23^{*}$ \\
\hline * Correlation is significant at the .01 level (2-tailed).
\end{tabular}

\section{Study 2: Method and results}

Study 2 tried to extend the finding from study 1 by investigating failures to suppress unwanted thoughts in an experiment along the lines of the White Bear paradigm (Wegner et al., 1987). In our experiment, we measured suppressive capability using highly emotional stimulus material. Thus, 40 randomly selected undergraduate students (24 women), who also participated in study 1, were invited to take part in study 2 that took place approximately 4 months later. Their mean age was 18.55 years $(S D=1.37$; range: 17 to 23 years). Participants were unaware of a relationship between the two studies. Study 2 was approved by the standing ethical committee of our faculty.

Participants were tested individually in our lab with a femalle experimenter present. First, the DES was administered. Then participants viewed an emotional video fragment depicting a severe, but non-fatal automobile accident. The fragment showed a car-rollover and has been used as evidence by the Dutch police in a court case and all participants saw it for the first time. Its duration was approximately $1 \mathrm{~min}$.

Subsequently, participants were instructed not to think about the film for a $5 \mathrm{~min}$ period (suppression phase). Following this, participants were allowed to think about anything including the video (expression phase). The expression phase also lasted $5 \mathrm{~min}$. Prior to each phase, participants were instructed to push a button in front of them every time they thought about the video. Key presses were monitored. Finally, participants were fully debriefed.

As all participants completed the questionnaires twice, we calculated the DES, DES-I", and DES-T taxon probability scores for both moments in time. DES (Cronbach 's $\alpha=.95$ ) and DES-T (Cronbach's $\alpha=83$ ) scores for these 40 participants at time 1, i.e., during study 1, were $19.51(S D=13.52)$ and $12.65(S D=12.48)$, respectively. At time 2, thus during study 2 , DES (Cronbach's $\alpha=.94)$ and DES-T (Cronbach's $\alpha=.81)$ scores were 16.14 $(S D=12.98)$ and $9.23(S D=12.26)$, respectively. Note that although the test-retest correlations for the $\operatorname{DES}(r=.81, p<.01)$ and the DES-T $(r=.73, p<.01)$ were significant, both DES $(t(39)=2.63, p<.05)$, and DES-T $(t(39)=2.53, p<.05)$ declined significantly from time 1 to 2 . 
Table 6.2. Spearman correlations between Dissociative Experiences Scale (DES), the Dissociative Experiences Scale Taxon total score (DES-T), Dissociative Experiences Scale Taxon probability (DES-T Taxon), and the thought suppression task in an undergraduate sample $(n=40)$. All questionnaires were administered twice; four months prior to and during the experiment (time $\mathbb{I}$ and time 2).

\begin{tabular}{cccccccccc} 
& & \multicolumn{2}{c}{ DES } & \multicolumn{2}{c}{ DES-T } & \multicolumn{2}{c}{ DES-T Taxon } & \multicolumn{2}{c}{ Thought } \\
& time & $\mathbf{1}$ & $\mathbf{2}$ & $\mathbf{1}$ & $\mathbf{2}$ & $\mathbf{1}$ & $\mathbf{2}$ & suppress & express \\
& $\mathbf{1}$ & - & $.84^{* *}$ & $.86^{* *}$ & $.84^{* *}$ & $.65^{* *}$ & $.61^{* *}$ & .15 & .20 \\
DES & 2 & & - & $.74^{* *}$ & $.91^{* *}$ & $.52^{* *}$ & $.71^{* *}$ & .04 & .14 \\
& 1 & & & - & $.82^{* *}$ & $.75^{* *}$ & $.53^{* *}$ & .31 & .12 \\
DES-T & 2 & & & & - & $.59^{* *}$ & $.73^{* *}$ & .22 & .15 \\
& 1 & & & & & - & $.44^{* *}$ & $.35^{*}$ & $.32^{*}$ \\
DES-T & 1 & & & & & - & .06 & .13 \\
Taxon & 2 & & & & & & & - & $.46^{*}$ \\
Thought & suppress & & & & & &
\end{tabular}

** Correlation is significant at the .01 level (2-tailed).

* Correlation is significant at the .05 level (2-tailed).

As the distribution of our measures was skewed, non-parametric correlations were calculated. Table 6.2 shows the Spearman correlation between DES, DES-T, DES$\mathrm{T}$ taxon probability, and target thought intrusions during thought suppression and expression. As can be seen in table 6.2, DES, DES-T, and DES-T taxon probabilities, as measured during study 2 , appear to be unrelated to frequency of target thoughts during suppression and expression phases. However, the correlation between the DES-T total score from the same participant obtained during study 1 and failures to suppress target thoughts during study 2 reaches borderline significance $(p=.06)$. As well, correlations between DES-T taxon probability ratings from the same participants, obtained during study 1 and both suppression deficits and the rebound during the expression period attained significance.

\section{Discussion}

The most important findings of our two studies can be catalogued as follows. To begin with, the relationship between dissociative experiences, as measured with the DES, and the suppression of unwanted thoughts, as measured with the WBSI, was replicated. We also showed that similar significant correlations with WBSI can be found, when one focuses on the more pathological manifestations of dissociation, as measured with DES$\mathrm{T}$ (Waller et al., 1996) and its taxon probability. In addition, failure to suppress target thoughts about an emotional video fragment was related only to the more pathological dissociative symptoms, as measured with DES-T and its taxon probability. Heightened scores on these parameters were also accompanied by a more prominent rebound effect during expression. However, these effects only attained significance when DES-T and DES-T taxon probabilities were derived from the data collected during the first test occasion, i.e., 4 months prior to the thought suppression experiment.

Using a behavioral measure of suppressive capabilities (i.e., thought suppression task; Wegner et al., 1987), the relationship between dissociative experiences and the suppression of unwanted thoughts (Muris \& Merckelbach, 1997; 
wan den Hout et al., 1996) could be replicated, at least for the pathological manifestation of dissociation. Our findings, then, illustrate that individuals exhibiting these particular dissociative symptoms, exhibit poor suppressive capabilities. This is in line with Hoeping and de Jong-Meyer (2003), who argue that the WBSI taps thought intrusions stemming from an inability to suppress target thoughts. The relatively poor ability to suppress unwanted thoughts exhibited by individuals high on the DES-T is consistent with research showing that disnuptions in executive (i.e., frontal) functioning (Cima et al. 2001), inhibitory processes (chapter 5), and cognitive efficiency (chapter 4) are associated with dissociation. In more general terms, they suggest that high dissociative individuals have difficulties with attentional control, which makes them susceptible to thought intrusions.

Another important aspect of our studies is the context sensitivity of the DES. Previous studies have noted that total DES scores of individuals may decline from one to the other testl occasion (see Merckelbach, Dekkers, Wessel, \& Roefs, 2003). Our finding that DES parameters obtained during the first but, not the second test occasion were related to intrusions of target thoughts is related to this phenomenon. At first sight, this is a rather counterintuitive finding; as research on the link between hypnosis and absorption indicates that administering a measure of absorption right after a measure of hypnosis inflates the correlation between these two measures (Council \& Kirsch, 1996). Similarly, the relationship between psi beliefs (e.g., extrasensory perception, ghosts) and dissociation is inflated when the two measures are presented together (Council, 1993). Council and Kirsch (1996, p. 30) propose that this is "due to a tendency for consistency in self-presentation." Thus, participants believe that a high score on a hypnosis or psi belief measure should go hand in hand with high dissociation scores. However, Council (1993) demonstrated that presenting two measures in the same context may also masquerade effects, such as the relationship between psi beliefs (e.g., extrasensory perception, ghosts) and psychiatric symptoms. This research showed that when certain items have pathological connotations they will less $\Downarrow$ ikely be endorsed by participants when their relationship can be inferred. In Council's (1993, p. 32) words "Failure to control for context may actually obscure significant relationships among variables." The data of study 2 can be interpreted along these lines. That is, dissociation soredropped when parterpantf were tested individully and they knew that DES scores could be linked to their person. This may help explain why the correlation between intrusions and pathological manifestations of dissociation was context dependent. Perhaps, then, participants exhibited a tendency to present themselves more favorable in the one-on-one situation in our laboratory than during the anonymous mass testing session. This tendency is coined supernormally by Cima el al. (2003). Our findings suggest that researchers using the DES are well-advised to reduce superinormality tendencies by informing participants that the data will be collected anonymously. One way in which this can be done is by creating a context where the participant feels minimally observed (e.g., leaving the room, while the participant is lilling in the questionnaires; not asking for names or student numbers). An even better alternative is measuring the frequency of dissociative experiences in a separate context, e.g. during a mass screening session.

Two shortcomings of our studies warrant comment. First, the data were collected in a healthy undergraduate sample. It may well be the case that correlations between DES and thought intrusions would have been much more impressive and consistent, had we collected data in clinical samples. Second, study 2 relied on a simple version of Wegner et al.'s (1987) thought suppression paradigm. Ideally, one would 
counterbalance suppression and expression phases and use either emotional or neutral target thoughts, but this, of course, would require a large-scale experiment.

To sum up, our research shows that dissociative experiences, notably their more pathological manifestations are related to thought intrusion. Our study also makes clear that researchers investigating dissociation have to be aware of the context dependency of the DES, an issue that is extensively discussed by Council (1993). Systematic studies addressing factors that lead to inflation or attenuation of relations between the DES and other measures are needed, as both can seriously threaten the validity of findings. 


\section{Part III: Dissociation, sleep, and cortical activity}

All the fanous moralists of olden days drew attention to the way in which certain happenings would leave indelible and distressing memories - memories to which the sufferer was continually retuming, and by which he was tormented by doy and night.

- Pierre Janet 


\section{Chapter 7: Subjective Sleep Experiences Are Related to Dissociation*}

\section{Abstract}

We examined the relationship between dissociative experiences as indexed by the Dissociative Experiences Scale (DES; Bernstein \& Putnam, 1986) and sleep- related experiences (e.g., nightmares, recurring dreams) as measured by the Iowa Sleep Experience Survey (ISES; Watson, 2001) in an undergraduate sample $(n=94)$. In addition, we studied the link between dissociative experiences and sleeping pattern as measured by the Morningness-Eveningness Questionnaire (MEQ; Home \& Östberg, 1976). In keeping with earlier work, we found that heightened levels of dissociation are related to a raised frequency of self-reported sleep disturbances. However, dissociation was not related to individual differences MEQ scores.

* Giesbrecht, T., \& Merckelbach, H. (2004). Subjective sleep experiences are related to dissociation. Personality and Individual Differences, 37, 1341-1345. 


\section{Introduction}

The marked influence of sleep deprivation on performance and alertness has been shown by numerous studies (Jewett et al., 1999). However, the issue of how individual differences in sleeping pattern and sleeping quality are related to traditional personality traits has received little attention. This is astonishing as individual differences in sleeping behavior (e.g., frequency of nightmares and lucid dreams) are stable over time and consistent across different contexts (see for a recent study, Watson, 2003b). Also, there are reliable and valid self-report measures that tap individual differences in such sleep-related experiences (Watson, 2003b).

Given their dreamlike properties, one might suspect a robust link between dissociative symptoms (e.g., derealization, depersonalisation, and annesia) and sleeprelated experiences. In a pioneering study relying on two large student samples, Watson (2001) demonstrated this relationship by showing that dissociative experiences symptoms as measured by the Dissociative Experiences Scale (DES; Bernstein \& Putnam, 1986) and sleep- and dream-related experiences as indexed by the Iowa Sleep Experiences Survey (ISES; Watson, 2001, 2003b) are, indeed, correlated with each other, with Pearson correlations circling around .50. On the basis of this finding, Watson (2001) speculated that dissociative symptoms might be fuelled by labile sleepwake cycles, such that individuals with these symptoms easily pass from normal waking mentation to dream-like states. Clearly, this research line offers a fresh perspective on the origins of dissociative symptoms, which are commonly understood as reflecting a defensive coping style caused by a history of childhood trauma (e.g., Merckelbach \& Muris, 2001). With this in mind, the current study made an attempt to replicate Watson's observation that dissociative and subjective sleep experiences are related to each other. In addition, we explored how dissociation relates to sleeping schedule as measured by the Morningness-Eveningness Questionnaire (MEQ; Horne \& Östberg, 1976). Morning type respondents prefer activities during the morning. In contrast, evening type respondents are characterized by a preference for evening activities. Eveningness is associated with irregularities in sleep-wake schedules, complaints of sleep debt and excessive daytime sleepiness (Gianotti, Cortessi, Sebastiani, \& Ottaviani, 2002). Thus, we expected that heightened dissociativity might be linked to eveningness.

\section{Method}

\section{Participants}

Participants were 94 undergraduate students enrolled at the Maastricht University. Sixty-four of them were women. Their mean age was 21.25 years ( $S D=2.16$; range: 18 to 27 years).

\section{Measures}

Dissociative Experiences Scale (DES; Crombach 's a $=.94$ ). The DES (Bernstein \& Putnam, 1986) is a self-report scale asking respondents to indicate on $100 \mathrm{~mm}$ visual analog scales to what extent they experience 28 dissociative phenomena in daily life. Examples of such phenomena include feelings of depersonalization, derealization, and psychogenic amnesia. A subset of 8 DES items forms the Dissociative Experiences Scale Taxon (DES-T; Cronbach's alpha $=.82$; Waller et al., 1996), which is thought to 
be especially sensitive to pathological dissociation. DES-T scores can be obtained by averaging across DES items 3, 5, 7, 8, 12, 13,22, and 27 (e.g., Eisen \& Carlson, 1998).

The lowa Sleep Experiences Survey (ISES; Cronbach's $\alpha=$.85). The ISES (Watson, $2001,2003 \mathrm{~b}$ ) consists of 18 questions asking the respondent to rate the frequency of various sleep- and dream-related experiences (e.g., "A dream helped me to solve a current problem or concern.", "Lying in bed, I sense the presence of someone who actually isn't there.") on a 7 point-Likert scale (anchors: $1=$ never; $7=$ several times a week). The ISES contains two separate subscales that measure general sleep experiences (15 items; Cronbach's $\alpha=.81$ ) and lucid dreaming ( 3 items; Cronbach's $\alpha=.77$ ), respectively. These subscales show a moderate correlation with $r$ 's circling around .40 , indicating that they measure distinct, but related constructs (Watson, 2001). A mean score can be obtained by averaging across all ISES items.

Morninghess-Eveningness Questionnaire (MEQ; Cronbach's alpha $=.82$; Horne \& Östberg, 1976). The MEQ is commonly used to classify individual differences in sleeping schedules of respondents. Based on their answers to items like "During the first half-hour after having woken in the morning, how tired do you feel?", respondents are categorized as belonging to one of the following five groups: definitive morningness, moderate morningness, intermediate, moderate eveningness, and definitive eveningness (Horne \& Östberg, 1976).

All questionnaires were Dutch translations and have been extensively used during prior research, which yielded results highly similar to studies relying on the original English versions.

\section{Results}

Mean DES, ISES, and MEQ scores were 16.13 $(S D=11.33), 2.66(S D=0.75)$, and $46.78(S D=9.09)$, respectively. It is worthy of note that the mean DES score corresponds with values that previous studies reported for student samples (e.g., Merckelbach et al., 2002). Table 7.1 presents Pearson correlations between DES, ISES, and MEQ scores. Correlations with the subscales of the ISES are also shown.

Dissociative experiences were significantly correlated with total ISES scores and scores on its general sleep experiences subscale. However, the correlation of DES with the lucid dream subscale of the ISES reached only borderline significance $(p=.05$; two-tailed), while that with Morningness-Eveningness fell short of significance. Interestingly, the two ISES subscales correlated significantly with each other, while none of the ISES parameters was related to Morningness-Eveningness. The correlational pattern for the DES-T followed that of the DES. Thus, DES-T was related to ISES scores, but not to MEQ scores. 
Table 7.1. Pearson correlations between the Dissociative Experiences Scale (DES), Morningness-Eveningness Questionaire (MEQ), and the lowa Sleep Experience (ISES) in an undergraduate sample $(n=94)$. Correlations involving the General Sleep Experiences Subscale of the ISES (ISES GS), the Iowa Sleep Experiences Lucid Dreaming Subscale of the ISES (ISES LD) and the Dissociative Experiences Scale Taxon (DES-T) are also shown.

\begin{tabular}{lccccc} 
& DES & DES-T & ISES & ISES GS & ISES LD \\
\hline DES & - & & & & \\
DES-T & $.87^{*}$ & - & & & \\
ISES & $.37^{*}$ & $.38^{*}$ & - & & \\
ISES GS & $.38^{*}$ & $.38^{*}$ & $.97^{*}$ & - & - \\
ISES LD & .23 & .24 & $.75^{*}$ & $.56^{*}$ & -12 \\
MEQ & -.05 & -.05 & .12 & -.09 & .12 \\
\hline
\end{tabular}

$* p<.01$ level (2-tailed).

\section{Discussion}

Our findings replicate those of Watson (2001) in that we found heightened levels of dissociation to be linked to reports of sleep phenomena like narcolepsy, vivid and unusual dreams, and nocturnal experiences as waking dreams and sensing the presence of someone who is not present. Interestingly, the more pathological manifestations of dissociation (e.g., the DES-T; Waller et al., 1996) were also found to be related to these sleep experiences. However, we did not find a relationship between dissociation and lucid dreaming or evening-versus morning-oriented sleep schedules. Apparently, the link between dissociation and sleeping is quite specific, which argues against the idea that our findings are the result of a reporting bias. However, due to our relatively small and homogenous (i.e., student sample) sample the aforementioned null findings have to be interpreted with caution and require replication in larger and more diverse samples. We will return to this issue in the next chapter.

These findings are important because they might provide a starting point for new research avenues into the study of dissociative experiences. The received view is that dissociation serves a defensive function and is the result of exposure to traumatic events (Merckelbach \& Muris, 2001). However, our results as well as those of Watson $(2001 ; 2003 \mathrm{~b})$ are consistent with the speculation that individual differences in sleeping behavior might underlie dissociative experiences. Thus, high dissociators might more readily pass from normal waking into dream-like states, which might produce dissociative episodes. Of course, with the correlations obtained in the current study, it is impossible to test such causal theories. Yet, they might inspire studies in which speculations about the causal antecedents of dissociation can be fairly directly tested. For example, to the extent that a labile sleep-wake cycle produces dissociation, one would expect that sleep deprivation enhances dissociative experiences. Also, one would expect that certain EEG parameters (e.g., slow wave activity) are related to individual differences in dissociation.

The comnection between sleep experiences and dissociation might also explain why dissociative phenomena are associated with both fantasy proneness and cognitive failures (Merckelbach \& Muris, 2001; Merckelbach et al., 2002). Germane to this issue are studies indicating that sleep deprivation leads to decrements in alertness and performance (e.g., Jewett et al., 1999). Therefore, studies on sleep disturbances might 
shed a new light on the robust correlations ranging from .43 (Merckelbach al., 1999) to .53 (Merckelbach et al, 2002) between dissociative experiences as measured by the DES and susceptibility to cognitive failures as measured by the Cognitive Failures Questionnaire (CFQ; Broadbent et al., 1982).

Another robust correlation is that between dissociation and fantasy proneness as measured by the Creative Experiences Questionaire (CEQ; Merckelbach et al., 2001), which varies from .48 (Merckelbach et al., 2000b) to .58 (Merckelbach et al., 1999). Fantasy proneness refers to a deep and extensive involvement in fantasy and daydreaming (Merckelbach et al., 2001) and has been linked to dream recall (Watson, 2001). Research on neural nets suggests that regular dreaming might increase the efficiency of neural information processing. By this view, Rapid Eye Movement Sleep prevents interference between different classes of information, which some have taken to imply that we "dream to reduce fantasy" (Crick \& Mitchison, 1995; p. 150). Thus, it is not too farfetched to speculate that disturbed dreaming patterns might promote rather than inhibit fantasies.

To sum up, then, the findings of Watson $(2001,2003 \mathrm{~b})$ and those of the current study might broaden our understanding of the nature of dissociative experiences by relating them to differences in sleeping behavior. However, one clear limitation of this research is its reliance on self-reports and non-clinical samples. Future research based on objective measures such as slow waves in resting $\mathbb{E E G}$ might underpin the correlation between dissociation and sleep experiences. 


\section{Chapter 8: Dreaming to reduce fantasy - Fantasy proneness, dissociation, and subjective sleep experiences}

\section{Abstract}

Fantasy promeness refers to an extensive involvement in fantasy and daydreaming. Previous studies have shown that fantasy proneness overlaps with dissociative tendencies, as measured with the Dissociative Experiences Scale. We tested the hypothesis that deviant sleep experiences form the critical link between fantasy proneness and dissociation. Undergraduate students $(n=205)$ completed the Creative Experiences Questionnaire, the Dissociative Experiences Scale, and the lowa Sleep Experiences Survey. Self-reports of sleep experiences, such as narcolepsy, vivid and unusual dreams, and deviant nocturnal experiences, but not lucid dreaming, were related to both fantasy proneness and dissociation. However, the relationship between fantasy proneness and dissociation was only partially accounted for by sleep experiences. This suggests that the overlap between fantasy proneness and dissociation, does not solely originate from deviant sleep experiences but also from other factors

\footnotetext{
"Giesbrecht, T., \& Merckelbach, H. (in press). Dreaming to reduce fantasy - Fantasy proneness, dissociation and subjective sleep experiences. Personality and Individual Differences.
} 


\section{Introduction}

Crick and Mitchison (1995, p. 150) said about the function of rapid eye movement (REM) sleep that "we dream to reduce fantasy." This hypothesis is based on the idea that REM sleep serves to increase the efficiency of the brain. Evidence for this idea comes from two different sources. Firstly, the Echidna (an Australian monotreme) possesses an abnormally large neocortex relative to its body, while showing no signs of REM sleep. This suggests that one function of REM sleep might be to store more information in a smaller space. Secondly, simulation experiments with neural nets indicate that random stimulation from the brain stem during REM sleep might prevent overlap of stored information by reducing interactions between related concepts (Gardner Medwin \& Kaul, 1995). This would reduce involuntary activation of related, but inappropriate concepts.

These findings have interesting implications for the study of individual differences in fantasy proneness. Fantasy proneness refers to a deep and extensive involvement in fantasy and daydreaming (Merckelbach et al., 2001). Speculations about how sleep and daydreaming might be related to each other have a long tradition in psychology that goes back to Sigmund Freud (1907/1906). Following that tradition, one could argue that individual differences in fantasy proneness might be fuelled by disruptions in REM sleep. However, while the robust effects of sleep deprivation on human performance have been extensively studied (e.g., Jewett et al., 1999;

Williamson, Feyer, Mattick, Friswell, \& Finlay Brown, 2001), individual differences in various sleep experiences, such as lucid dreaming, narcolepsy, and other nocturnal experiences have not received much attention. This is surprising, as individual differences in sleeping behavior are stable over time and different contexts (Watson, $2003 \mathrm{~b})$. In addition, there are reliable and valid self-report measures that tap individual differences in sleep-related behavior (Watson, 2001). One important exception is a pioneering study by Watson (2001), who explicitly looked at the relationship between sleep experiences and dissociative symptoms such as depersonalization, absorption, derealization, and memory problems. In two large samples, Watson (2001) demonstrated that sleep-related experiences, as measured by the lowa Sleep Experiences Scale (ISES; Watson, 2001, 2003b), are related to dissociative symptoms, as indexed by the Dissociative Experiences Scale (DES; Bernstein \& Putnam, 1986) with Pearson correlations circling around .50. This is an important finding, because it might shed a new light on the origins of dissociative symptoms.

It is a well-established fact that dissociative symptoms have a relatively high frequency in the general population (Gershuny \& Thayer, 1999). Common explanations for their origins have focused on early traumatic experiences as the antecedents of dissociation (Gast et al., 2001). However, recent research has made it clear that early traumatic experiences cannot account for the high prevalence of various dissociative symptoms. There are some indications that traumatic experiences might be connected to a special subgroup of dissociative symptoms involving severe memory problems (i.e., amnesia), depersonalization, and derealization (Waller et al., 1996). This pathological subgroup of symptoms has been termed the DES-T (Waller et al., 1996). Interestingly enough, even these more pathollogical manifestations of dissociation, appear to be related to disturbed sleep patterns (see chapter 7). 
Another robust correlate of dissociative experiences is fantasy proneness (Merckelbach et al., 2002). The substantial overlap between these two constructs implies that they define a common domain. In this study, we wanted to test whether disturbed sleep experiences form the critical factor that ties together (i.e, mediates) dissociation and fantasy proneness. If it could be shown that the overlap between dissociation and fantasy proneness critically depends on disturbed: sleep patterns, then this would open new theoretical perspectives.

\section{Method}

\section{Participants}

Participants were 205 undergraduate students (140 women) enrolled at the Maastricht University. Their mean age was 19.4 years ( $\mathrm{SD}=1.75$; range: 17 to 26 years). Participants were compensated through a small amount of money. The study was approved by the local ethical committee. The questionnaires were completed in one mass testing session at the beginning of the semester.

\section{Measures}

Creative Experiences Questionnaive (CEQ; Cronbach's $\alpha=68$ ). The CEQ is an index of fantasy proneness, asking the respondent to state whether they agree with 25 statements concerning either developmental antecedents of fantasy pronemess, profound involvement in fantasy and daydreaming, or consequences of daydreaming. Sample items are: "I can recall many occurrences before the age of three.", "I spent more than half of the day fantasizing and daydreaming." Merckelbach et al. (2001) reported adequate test-retest stability, internal consistency, and concurrent validify for this instrument.

Dissociative Experiences Scale (DES; Cronbach's $\alpha=90$ ). The DES (Bernstein \& Putnam, 1986) is a 28-item self-report scale that asks the respondent to indicate the frequency of various dissociative experiences, such as derealization, depersonalization, and psychogenic amnesia on 100-mm visual analogue scales (VAS). A sample item is "Some people have the experience of looking in a mirror and not recognizing themselves. Mark the line to show what percentage of the time this happens to you." The anchors of the 100-mm VAS are 0 (never) and 100 (always). In a meta-analytic study, Van IJzendoorn and Schuengel (1996) provide evidence for the sound psychometric properties of the DES. The DES exhibits high internal consistency, while test-retest correlations range from .74 to .84 . A subset of 8 DES-items forms the Dissociative Experiences Scale Taxon (DES-T; Cronbach's $\alpha=.75$; Waller et al., 1996), which is thought to be especially sensitive to pathological dissociation. DES-T total score can be obtained by averaging across DES items $3,5,7,8,12,13,22$, and 27 (e.g., Eisen \& Carlson, 1998).

Iowa Sleep Experiences Survey (ISES; Cronbach's $\alpha=.80$ ). The ISES (Watson, 2001) consists of 18 questions asking the respondent to rate the frequency of various sleepand dream-related experiences on a 7 point-Likert scale (anchors: $1=$ never, $7=$ several times a week). The ISES consists of 2 separate subscales, which measure general sleep experiences (Cronbach"s $\alpha=.79$, e.g.* "I have recurring dreams.") and lucid dreaming (Cronbach's $\alpha=.73$, e.g., "I am aware that I am dreaming, even as I dream"), 
respectively. Whereas the general sleep experiences subscale taps symptoms of narcolepsy, wivid and unusual dreams, and other nocturnal experiences, the lucid dreaming subscale consists of several items about knowing that you are dreaming while still being asleep. Previous research by Watson (2001) shows that these two subscales are moderately correlated with $r$ 's circling around .40 , indicating that they measure distinct, but related constructs. Watson $(2001 ; 20036)$ obtained both evidence for the convergent validity and acceptable internal consistency of the ISES. Note that there is no overlap in content between the CEQ and ISES items.

All questionnaires were Dutch translations and have been extensively used during prior research, which yielded results highly similar to studies relying on the original English versions.

\section{Results}

Mean CEQ, DES, DES-T, and ISES score were $7.39(S D=3.53), 18.61(S D=10.63)$, $10.97(S D=9.87)$, and $2.93(S D=0.73)$, respectively. These scores closely correspond with values reported by previous studies for student samples (Merckelbach et al., 2002; Merckelbach et al., 2000a). Table 8.1 shows Pearson product-moment correlations between the CEQ, DES, DES-T, and the ISES. In addition, correlations with these measures and the two subscales of the ISES are presented.

Table 8. 1. Pearson product-moment correllations between the Dissociative Experiences Scale (DES), the Dissociative Experiences Scale Taxon (DES-T), Iowa Sleep Experiences Scale (ISES), the General Sleep Experiences Subscale of the ISES (ISES GS), the ISES Lucid dreaming subscale (ISES LD), and the Creative Experiences Scale (CEQ) in an undergraduate sample $(n=205)$,

\section{DES DES-T ISES ISES GS ISES LD}

$\begin{array}{llllll}\text { DES } & - & & & & \\ \text { DES-T } & .84^{*} & - & & & \\ \text { ISES } & .33^{*} & .34^{*} & - & & \\ \text { ISES GS } & .35^{*} & .35^{*} & .95^{*} & - & \\ \text { ISES LD } & .08 & 0.1 & .57^{*} & .28^{*} & - \\ \text { CEQ } & .44^{*} & .41^{*} & .36^{*} & .38^{*} & 0.1\end{array}$

* Correlation is significant at the .01 level (2-tailed).

Fantasy proneness, as measured with the CEQ, was significantly related to both dissociation, as measured with the DES, and sleep experiences, as measured with the ISES. In addition, fantasy proneness displayed a significant correlation with the general sleep experiences subscale of the ISES, while the correlation between fantasy proneness and the lucid dreaming subscale of the ISES remained non-significant. For the association between DES-T and ISES subscales, a comparable correlational pattern was found.

The mediational effect of sleep-related experiences on the relationship between dissociation and fantasy proneness was examined using the approach outlined by Baron and Kenny (1986). Firstly, we tested the unmediated model, consisting of the relationship between dissociation and fantasy proneness. Dissociative experiences, as 
measured by the DES, were found to significantly predict fantasy proneness, as measured by the CEQ, $B=0.15, \beta=0.44, t=7.30, p<.01$. Next; we tested the mediated model consisting of dissociative experiences, fantasy proneness, and sleep experiences. Dissociative experiences predicted sleep experiences, as measured by the ISES, $B=0.02, \beta=0.34, t=5.45, p<.01$, whereas sleep experiences predicted fantasy proneness, $B=1.30, \beta=0.27, t=4.33, p<.01$. In addition, though still being significant, the predictive power of dissociation with regard to fantasy proneness decreased, as compared to the unmediated model. It is, however, not sufficient to show that the relation between the predictor and the outcome is reduced when the mediator is added to the model (Frazier et al., 2004). Therefore, we calculated Sobel's (1982) test. This test indicated that the relationship between dissociation and fantasy proneness is partially mediated through sleep experiences, $Z=3.39, p<.01$, with $21 \%$ of the relation between dissociation and fantasy proneness being mediated by sleep experiences. A mediator analysis investigating the influence of sleep experiences on the relation between the DES-T and fantasy proneness yielded similar results with Sobel's test indicating a significant mediator effect for these sleep experiences $(Z=3.46, p<$ $.01)$.

\section{Discussion}

Our findings can be catalogued as follows. Firstly, in line with previous research (Merckelbach et al., 2000a; Merckelbach et al., 1999), dissociation and fantasy proneness correlated with each other. Secondly, we replicated the relationship between dissociation and sleep experiences (Watson, 2001, 2003b; chapter 7). Thirdly, fantasy proneness was related to sleep experiences, as measured by the ISES and its general sleep subscale, but not the frequency of lucid dreaming. Fourthly, the close connection between dissociation and fantasy proneness appeared to be mediated by sleep experiences. This mediation was, however, only partial and could not fully account for the relationship between dissociation and fantasy proneness. Interestingly, the more pathological manifestations of dissociation (i.e., the DES-T) displayed a similar pattern of results in our study. That is, to some extent, the overlap between DES-T and fantasy proneness was meditated by deviant sleep experiences, but this factor could not fully for account the overlap.

Our data suggests that fantasy proneness is, indeed, related to various sleeprelated experiences. The link between fantasy proneness and these sleep experiences is, apparently, quite specific, as fantasy proneness was only related to general sleep experiences, such as narcolepsy, viwid and unusual dreams, and nocturnal experiences. However, fantasy proneness was unrelated to self-reports of lucid dreaming. This finding rules out a positive response bias (i.e., a tendency to endorse all sorts of atypical symptoms), which is sometimes associated with fantasy proneness (Merckelbach et al., 2000a).

Although we found a mediational effect of sleep experiences on the relationship between dissociation and fantasy proneness, these sleep experiences could only explain part of the overlap. This is shown by the fact that the link between dissociation and fantasy proneness remained intact even when correcting for the contribution of sleep experiences (i.e., partial mediation; Frazier et al., 2004). Perhaps, fantasy proneness is more closely related to involuntary activation of related concepts due to disturbance in REM sleep, whereas dissociation is characterized by shifts from normal waking to dream-like states resulting from stage I sleep intrusions during 
daytime (Watson, 2001). Of course, these speculations warrant further study. In particular, studies linking EEG parameters of waking and sleeping to dissociation and fantasy proneness might be informative.

To conclude, our findings might broaden our understanding of the origins of both dissociation and fantasy proneness by relating these traits to differences in sleep experiences, such as nightmares and narcolepsy. In addition, links with sleep experiences were not limited to the benign manifestations of dissociation, but also extended to its more malign manifestations, as indexed by the DES-T. Our findings should, however, be interpreted with caution, as they are solely based on self-report. That is why research using polysomnography to underpin our and Watson"s findings $(2001,2003 \mathrm{~b})$ is needed. 


\section{Chapter 9: Dissociation, resting EEG, and subjective sleep experiences in undergraduates}

\section{Abstract}

In this study, we explored whether individual differences in dissociation are related to certain resting EEG parameters. Baseline EEG with eyes open and closed was recorded in an undergraduate sample $(n=67)$. Cortical power in the $\alpha$-range was inversely related to dissociative symptoms, as measured by the Dissociative Experiences Scale (DES), while both $\delta$ - and $\theta$-power where positively related to dissociation. However, sleep experiences, as indexed by the lowa Sleep Experiences Survey, were unrelated to resting EEG characteristics. We propose that suppression in the $\alpha$-band and raised levels of $\theta$-activity that are typical for high dissociators, might help to explain why dissociative symptoms are accompanied by attentional and memory deficits.

* Giesbrecht, T., Jongen, E., Smulders, F., \& Merckelbach, H. (in press). Dissociation, cortical activity, and sleep. Jonmal of Nervous and Mental Disease. 


\section{Introduction}

Dissociative experiences, like depersonalization, absorption, and derealization, appear to be quite common in the general population. Studies indicate that as much as $80 \%$ to $90 \%$ of the respondents report having these experiences at least some of the time (Gershuny \& Thayer, 1999). Bernstein and Putnam (1986, p. 727) define dissociation as "a lack of normal integration of thoughts, feelings, and experiences into the stream of consciousness", emphasizing the role of memory and attentional deficits. Likewise, Freyd et al. (1998, p. S91) state that "it would seem that fundamental basic cognitive mechanisms of memory and attention are implicated for the involvement in the phenomenon of dissociation." The relatively few studies that followed this lead found that dissociation is, indeed, related to disruptions in memory and attention. For instance, Candel et al. (2003) and we ourselves (chapter 2) noted that elevated scores on the Dissociative Experiences Scale (DES; Bernstein \& Putnam, 1986) were related to the creation of commission errors in memory. This finding is in line with Merckelbach, Muris, Horselenberg et al. (2000a), who reported that dissociative symptoms were related to the creation of pseudo-memories. Their participants watched 40 slides. Twenty of them were photographs of common objects or situations, while 20 others were short paragraphs, which described a scene or an object. After they had seen the slides, participants were subjected to a surprise old-new recognition task. Relative to control participants, participants with heightened levels of dissociative symptoms (i.e., high dissociators) were more likely to claim that they had seen the new slides (see also Hyman \& Billings, 1998).

A related area of research has focused on the link between attention and dissociative symptoms. Studies in this field have consistently found that high dissociators exhibit subtle disruptions in attention. For example, DePrince and Freyd (1999) reported that high levels of dissociation were accompanied by a slowing of reaction times on a selective attention Stroop (1.935) task. Other studies found some tentative evidence that dissociative tendencies are related to subtle disruptions in executive functioning (Cima et al., 2001; chapter 5).

In sum, research indicates that dissociative experiences go along with cognitive disruptions (e.g., Freyd et al., 1998; chapter 4). One would expect that this would have led to attempts to relate dissociation to neurophysiological manifestations of cognitive functioning. However, to the best of our knowledge, so far no such attempts have been made (but see Russ, Campbell, Kakuma, Harrison, \& Zanine, 1999). This is surprising as there is an extensive literature on how, for example, tonic and phasic changes in EEG power are linked to attention and memory (see for a review: Klimesch, 1999). A case in point are studies suggesting that $\alpha$-power is an indicator of speed of cognitive and in particular memory performance (Klimesch, Doppelmayr, Schimke, \& Pachinger, 1996; Surwillo, 1961, 1963a, 1963b). As early as 1961, Surwillo noted that relative to controls, individuals with thigh $\alpha$-frequency exhibit faster reaction times (see also Klimesch et al., 1996). Klimesch (1999; see also Vogt, Klimesch, \& Doppelmayr, 1998) ascribed the superior reaction time performance of individuals with relatively high power in the $\alpha$-range and low power in the $\theta$-range to the ease with which they can retrieve memories. In addition, research indicates that various neurological deficits (e.g., Alzheimer's disease), are related to a reduction in the $\alpha$-power range, while heightened levels of $\theta$-power are associated with poor performance during sustained attention tasks (Beatty, Greenberg, Deibler, \& O'Hanlon, 1974). With this in mind, one would predict 
that that heightened levels of dissociation would be accompanied by increased (tomic) levels of $\theta$ and decreased levels of tonic $\alpha$-power.

Individual differences in EEG power might be the result of fatigue and deviations in circadian rhythms (Klimesch, Vogt, \& Doppelmayr 1999$)$. The effects of these two factors on EEG power are well-documented. For example, since the pioneering study of Dement and Kleitman (1957), it is known that during the transition from alert wakefulness to sleep onset (i.e., the hypnagogic state), a-power decreases and $\theta$ - and $\delta$-power increase. These findings have been replicated in a more recent study (Tanaka, Hayashi, \& Hori, 1997). In line with previous findings, Cajochen et al. (in Klimesch, 1999) have shown that administration of melatonin, which regulates waking and sleeping and increases sleepiness, leads to an increase in the $\theta$ band and a decrease in the $\alpha$-band.

Interestingly, Watson $(2001 ; 2003 \mathrm{~b})$ recently proposed that dissociative experiences possess dream-like properties, which might be fuelled by a labile sleepwake cycle. This would imply that individuals having frequent dissociative experiences more easily pass from normal waking mentation to dream-like states. Support for this line of reasoning comes from studies showing that dissociative experiences, as measured with the DES (Bernstein \& Putnam, 1986) are, indeed, related to various sleeping experiences, as measured with the lowa Sleep Experiences Survey (ISES; Watson, 2001). In a recent study, we (Giesbrecht \& Merckelbach, 2004) replicated this finding and demonstrated that the more pathological manifestations of dissociation, as measured with the DES-T (Waller et al., 1996), are also related to sleep disturbances. That the relationship between sleep experiences and dissociation is not limited to this particular self-report scale of sleep experiences (i.e., ISES), was demonstrated by Levin and Fireman (2002) and Agargun (2003a), who noted a substantial overlap between dissociative experiences and nightmare frequency.

On the basis of the literature reviewed above, we hypothesized that dissociation and subjective slleep experiences are related to heightened $\theta$ and lowered $\alpha-$ power. The current study was a first attempt to test this idea.

\section{Method}

\section{Participants}

Participants were invited to take part in an electroencephalographic study on "attention and memory." They were recruited through a bulletin board at the Faculty of Psychology, Maastricht University. Participants were 67 right-handed undergraduate students (59 women) at Maastricht University, with a mean age of 21.1 years (range: $18-31, S D=2.68$ ). Hand preference was determined on the basis of self-report. The study was approved by the local ethical committee and all participants were naive as to the purpose of the study so as to minimize unwanted expectancy effects (Council, 1993). In return for their participation, they were given a small financial compensation.

\section{Self-report measures}

Dissociative Experiences Scale (DES; Cronbach's $\alpha=90$ ). Bernstein and Putnam $(1986$, p. 727$)$ developed the DES as "a means of reliably measuring dissociation in normal and clinical populations." This instrument consists of 28 items that ask the respondent to indicate the frequency of various dissociative experiences, suchl as derealization, depersonalization, and psychogenic amnesia, on $100-\mathrm{mm}$ visual analogue 
scales (anchors: $0=$ never; $100=$ always). A sample item is: "Some people have the experience of looking in a mirror and not recognizing themselves. Mark the line to show what percentage of the time this happens to you." In a meta-analytic study, Van IJzendoom and Schuengel (1996) provided evidence for the sound psychometric properties of the DES. The DES possesses high internal consistency and test-retest correlations range from .74 to .84 .

A subset of 8 DES items forms the Dissociative Experiences Scale Taxon (DES-T; Waller et al., 1996; Cronbach's alpha $=.80$ ), which is thought to be especially sensitive to pathological manifestations of dissociation. DES-T total score can be obtained by averaging across DES items $3,5,7,8,12,13,22$, and 27 (e.g., Eisen \& Carlson, 1998). The DES-T pretends to offer an index of clinically relevant dissociation that is largely orthogonal of non-pathological dissociative experiences (i.e., absorption). However, recent research by Levin and Spei (2003) indicates that this effort was only partially successful. Employing the DES-T eliminates some, but certainly not all of the overlap with imaginative involvement measures.

\section{lowa Sleep Experiences Survey (ISES; Cronbach's $\alpha=.85$ ). The ISES (Watson, 2001)} consists of 18 questions asking the respondent to rate the frequency of various sleepand dream-related experiences on a 7 point-Likert scale (anchors: $1=$ never; $7=$ several times a week). The ISES consists of 2 separate subscales which measure general sleep experiences (Cronbach's $\alpha=.83$, e.g., "I have recurring dreams.") and lucid dreaming (Cronbach's $\alpha=.79$, e.g., "I am aware that I am dreaming, even as I dream"), respectively. The general sleep experiences subscale taps symptoms of narcolepsy, vivid and unusual dreams, and other remarkable sleep experiences. The lucid dreaming subscalle consists of several items that refer to the situation that one is aware of dreaming while still being asleep. Previous research by Watson (2001) shows that these two subscales are moderately correlated with each other, $r$ 's being in the order of about .40 , indicating that they measure distinct, but related constructs. Watson $(2001 ; 2003 \mathrm{~b})$ obtained evidence for the convergent validity and internal consistency of the ISES.

\section{Procedure}

On arrival, participants were informed that EEG was to be measured. They were shown the control room with its equipment and the sound-attenuated testing room. After they gave informed consent, participants completed the Dissociative Experiences Scale (DES; Bernstein \& Putnam, 1986) and the lowa Sleep Experiences Survey (ISES; Watson, 2001), while they were fitted with a stretch EEG cap. Next, participants were brought to a sound-attenuated lab room, where two periods of baseline resting EEG, one with eyes open and one with eyes closed, were recorded. During the eyes open condition, participants were instructed to fixate a cross in the middle of a computer screen so as to minimize eye movements. The order of these two conditions was counterbalanced across participants. Following this, participants completed an unrelated cognitive task, the result of which will be reported elsewhere. Finally, participants were thanked and fully debriefed.

\section{EEG recording}

Using Neuroscan Synamps and Neuroscan Scan 4.3 software (NeuroSoft Inc., 2004), electroencephalographic (EEG) activity was recorded continuously during two periods of $3 \mathrm{~min}$ with an twenty-eight channel stretch-lycra EEG cap (Quik-Cap) containing tin 
electrodes placed according to the International 10-20 System. Electrical contact between electrodes and the participant's head was made with conducting gel, while positioning was achieved by using known anatomical landmarks. The montage included 5 midline sites ( $\mathrm{Fz}, \mathrm{FCz}, \mathrm{Cz}, \mathrm{Pz}, \mathrm{Oz}$ ) and 23 sites over each hemisphere (FP1/FP2, F3/F4, F7/F8, FC5/FC6, C3/C4, CP5/CP6, P3/P4, P7/P8, P9/P10, PO7/PO8, O1/O2 and the right mastoid). The left mastoid (A1) was used as the reference for all electrodes and AFz served as ground electrode. To facilitate artifact-correction of the EEG, tin electrodes were used to bipolarly record the vertical (above and below the left eye) and horizontal (at outer canthi of both eyes) electrooculogram (EOG). The EEG electrodes were re-referenced offline to the average of right and left mastoids (Hagemann, 2004). All electrode impedances were kept below $5 \mathrm{k} \Omega$. EEG and EOG were digitally refiltered with a bandpass of $0.05-100 \mathrm{~Hz}$. Digitization rate and gain were 500 and 1000 $\mathrm{Hz}$, respectively, and no notch filter was applied. Allen, Coan, and Nazarian (2004) pointed out that at least 2 min of continuous EEG recording are necessary, while longer periods add little to improve the internal consistency of the signal. Accordingly, we chose to record two periods of baseline EEG lasting 3 minutes.

\section{Data reduction and analysis}

Ocular activity was removed using a regression procedure (Semlitsch, Anderer, Schuster, \& Presslich, 1986). Next, the continuous signal was epoched in chunks, 1024 samples in duration, that overlapped $75 \%$ to compensate for loss of data (Tomarken, Davidson, Wheeler, \& Kinney, 1992). Epochs with signals exceeding $1 /-100 \mu \mathrm{V}$ were rejected. Artifact free epochs were subjected to a Fast Fourier Transformation (FFT). To eliminate interindividual variance in absolute EEG power, we normalized spectra by dividing each frequency step (of $0.5 \mathrm{~Hz}$ ) by the mean power, thereby expressing them as a percentage of mean power (see Vogt et al., 1998). Power spectra were normalized for each participant, condition (open vs. closed), and lead.

\section{Results}

\section{Individual differences measures}

Mean DES, DES-T, and ISES scores were $15.48(S D=9.41), 9.00(S D=10.02)$, and $2.81(S D=0.75)$, respectively. These values closely correspond to earlier findings in Dutch student populations (Merckelbach et al., 2002; chapter 7). Table 8.1 shows the correlation between the DES and the ISES. Correlations between the subseales are also presented.

As can be seen, there was a significant correlation between DES and ISES. As well the correlation between DES and the general sleep experiences subscale was significant. However, the correlation between the DES and the lucid dreaming subscale fell short of significance. The same pattern holds for the DES-T. This subscale correlated moderately with the ISES and its general sleep experiences subscale, but not with the lucid dreaming subscale. 
Table 8. 1. Pearson product-moment correlations between Dissociative Experiences Scale (DES), Dissociative Experiencess Scale Taxon score (DES-T), the Iowa Sleep Experiences Survey (ISES), the ISES general sleep (ISES GS), and the ISES lucid dreaming (ISES LD) subscale in an undergraduate sample $(n=67)$.

\begin{tabular}{lllll} 
& DES & DES-T & ISES & ISES GS \\
\hline DES & - & & & \\
DES-T & $.88^{*}$ & $*$ & & \\
ISES & $.49^{*}$ & $.41^{*}$ & - & - \\
ISES GS & $.55^{*}$ & $.47^{*}$ & $.97^{*}$ & $.41^{*}$ \\
ISES LD & .09 & .05 & $.63^{*}$ & \\
\hline
\end{tabular}

* Correlation is significant at the 01 level (2-tailed).
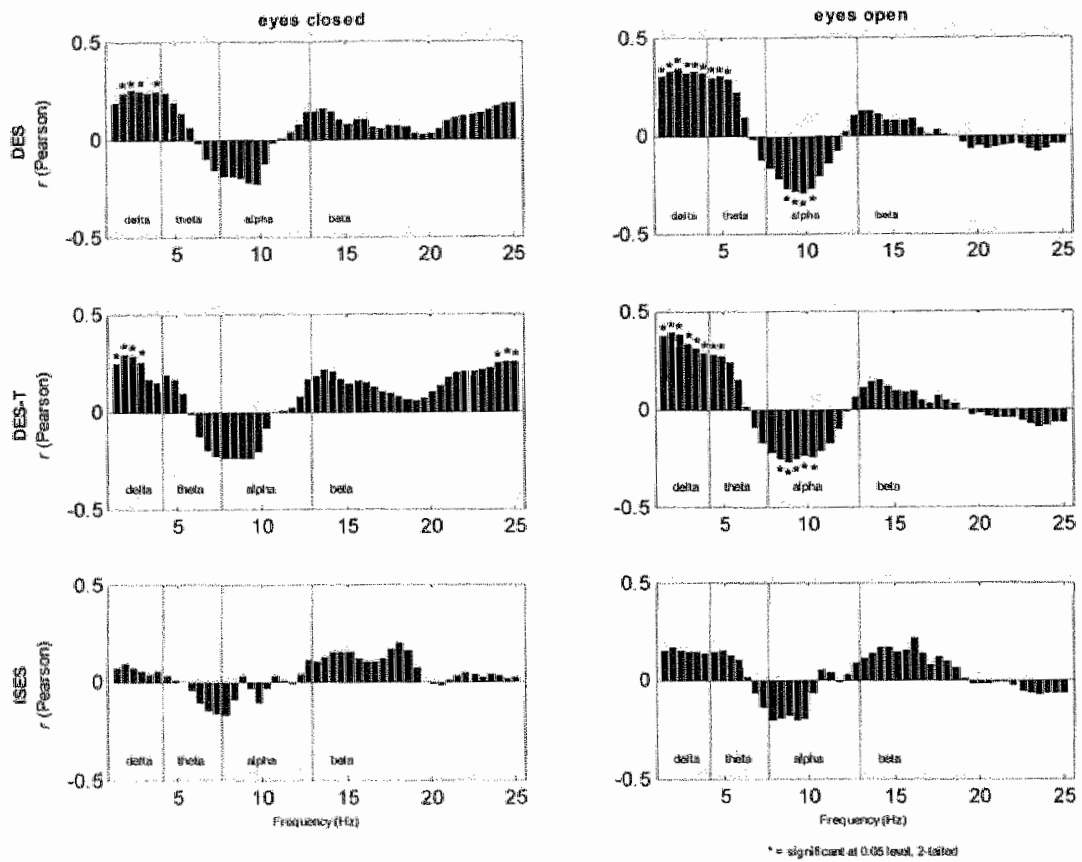

Figure 9.1. Bars represent Pearson correlation coefficients between Dissociative Experiences Scale (DES), Dissociative Experiences Scale Taxon (DES-T), and the Iowa Sleep Experiences Survey (ISES) and the normalized power averaged across all leads for each $0.50 \mathrm{~Hz}$ frequency step in the resting EEG in an undergraduate sample ( $n=$ 67).

\section{EEG power spectrum}

Klimesch ( 1999, p. 172) advocates the abolishment of traditional frequency bands and stated that the "use of fixed frequency bands does not seem justified", as it may masquerade effects. Following his recommendation, we calculated Pearson product 
moment correlations between DES, DES-T, ISES, and each frequency step of the normalized power averaged across all leads. Data were analyzed separately for eyes open and closed. Correlations are depicted in figure 9.1.

These analyses indicate that, especially in the eyes open condition, both DES and DES-T are related to reduced power in the $\alpha$-range and heightened power in both the $\theta_{-}$and the $\delta$-range. That these correlations are not limited to a certain area of the cortex is shown by the fact that a similar pattern of results evolved for all three areas (i.e., frontal, central, posterior), separately. That is, we calculated correlations between $\mathrm{DES}$ and normalized power for each frequency step in the three regions, frontal (Fpl, $\mathrm{Fp} 2, \mathrm{~F} 7, \mathrm{F3}, \mathrm{Fz}, \mathrm{F} 4, \mathrm{~F} 8)$, central $(\mathrm{C} 3, \mathrm{Cz}, \mathrm{C4}, \mathrm{FCz}, \mathrm{FC} 5, \mathrm{FC} 6, \mathrm{CP} 5, \mathrm{CP} 6)$, and posterior (P9, P7, P3, Pz, P4, P8, P10, PO7, O1, Oz, O2, PO8), but the basic pattern is similar. It is unlikely that the current results were systematically biased by eye movenents. Firstly, participants were instructed to fixate a cross during the eyes open condition. Secondly, and more importantly, artifacts caused by eye movements follow a distinct pattern, such that one would expect EEG effects due to eye movements to be most pronounced at frontal sites, while being weakest at posterior sites. Our results, however, do not follow this pattern.

We found no significant correlations between sleep experiences, as indexed with the ISES, and normalized power of the EEG.

\section{Discussion}

To our knowledge, our study was the first attempt to relate dissociative experiences and sleep experiences to a sleep-relevant measure that is independent of self-report. In line with previous research (chapter 7, 8; Watson, 2001, 2003b), we found individual differences in dissociative experiences to be related to self-reports of sleep-related experiences, but not of lucid dreaming. The DES-T followed a similar pattern. While DES and DES-T were related to suppression in the $\alpha$-power range and heightened levels in the slow wave power range (i.e., $\delta$ and $\theta$ ), the ISES was unrelated to cortical activity. The relationship between dissociation on the one hand, and both $\alpha$-power suppression and $\theta$-power increase on the other hand, was evident for all three cortical areas of interest.

Dissociative experiences were related to general sleep-related experiences, but not to lucid dreaming. Apparently, the link between dissociation and general sleep experiences is quite specific, which argues against the idea that the administration of these two measures within a single session might have led to spurious expectancy effects (Council 1993). In addition, in a recent study (Giesbrecht \& Merckelbach, in press), we ruled out report bias as another potential explanation for the link between dissociative symptoms and unusual sleep-experiences.

Our finding that dissociative experiences were unrelated to lucid dreaming is consistent with other research employing the ISES (Watson, 2001). Although more research is needed, we believe that the most plausible interpretation of the link between dissociative symptoms and general sleep experiences is that dissociative individuals have "thin boundaries" between the various stages of the sleep-wake cycle, allowing them to alternate between these stages effortlessly, which could be the origin of various dissociative symptoms (Watson, 2001). Lucid dreaming, however, is not affected by these "thin boundaries." Rather, this phenomenon represents "a cognitive skill that cam be increased by attentional and mnemonic techniques learned when awake" (Blagrove \& Hartnell, 2000, p. 42). 
In the present study, we failed to find supportive evidence for the idea that sleep experiences, as indexed by the ISES, are linked to baseline cortical activity. However, this null-finding has to be interpreted with caution given of our limited sample size. Furthermore, our study relied on a rather homogenous student sample and, therefore, comparing patient and healthy samples with and without prominent sleep disruptions would provide a more powerful test of the idea that dissociation, sleep disturbances, and cortical activity are intimately related. Likewise, inducing sleep disturbance artificially by means of sleep deprivation and studying its influence on state dissociation might also yield important insights.

Our finding that dissociation was related to suppression of power in the $\alpha$ range to some extent differs from the only other study (Russ et al., 1999) that looked at how dissociation relates to cortical activity. This study examined dissociation and background EEG during a cold pressor task (CPT) in a sample of female borderline personality disorder patients, depressed patients, and controls. The author found dissociative experiences to be positively related only to $\theta$-power. While we were able to replicate this particular aspect, at least in our undergraduate sample, dissociation was also related to lowered $\alpha$-power and raised $\delta$-power. This discrepancy might have to do with the specific sample characteristics in the Russ et al. (1999) study, notably their reliance on a mixed clinical sample. Another and more technical point is their use of fixed frequency bands, which might have blurred the negative association between $\alpha-$ power and dissociation (see Klimesch, 1999).

A number of limitations of our study deserve some comment. To begin with, dissociation overlaps with personality traits, such as absorption (Eisen \& Carlson, 1998), fantasy proneness (Merckelbach et al., 2005; Merckelbach et al., 2000a), suggestibility (Wolfradt \& Meyer, 1998), schizotypy (Watson, 2001), hypnotisability (Putnam, Helmers, Horowitz, \& Trickett, 1995), and self-reported history of trauma (Gast et al., 2001). In the current study, we did not assess these personality traits. Thus, we cannot rule out the possibility that these traits contribute in an important way to the dissociation-EEG pattern found in the current study. Clearly, this issue warrants further study. Secondly, our sample consisted primarily of women, which precluded a separate analysis for men and women. Thirdly, all participants were well adjusted undergraduates. While our participants differed widely in their self-reported frequency of dissociative experience, with DES scores ranging from 0 to 45 , Waller et all. (1996) suggest that one has to distinguish between two qualitatively different types of dissociation. These authors emphasize that non-pathological dissociative experiences, such as absorption, are quite common in the general population, while pathological manifestations of dissociation, like depersonalization and derealization, have a lower prevalence. Although we employed the DES-T to tap the pathological aspects of dissociation, one has to acknowledge that this index overlaps with non-pathological manifestations of dissociation (Levin \& Spei, 2003). Therefore, it remains possible that our sample exhibits a qualitatively different type of dissociative experiences in comparison to clinical samples. Hence, replication and extension of our findings, especially in clinical samples suffering from severe dissociative symptoms, while controlling for the aforementioned factors, would be highly informative.

Nevertheless, the fact that we found that dissociative experiences were linked to lowered $\alpha$-power and raised $\delta$ - and $\theta$-power might broaden our understanding of the nature of dissociative experiences. Germane to this issue are studies on how individual differences in tonic $\alpha$ - and $\theta$-power affect memory performance. For example, some studies noted a decrease in power in the upper $\alpha$-range in elderly participants (Klimesch, 
Vogt, \& Doppelmayr, 2000), notably patients with Alzheimer"s disease (Besthorn et al., 1997). Similarly, a reduction in a-power is characteristic for pre-school age children with neurological deficits (Schmid, Tirsch, \& Reitmeir, 1997) and patients with brain diseases that are known to impair memory performance (Klimesch, 1996).

The association between memory performance and $\alpha$ - and $\theta$-power might shed some light on a number of curious phenomena that co-vary with dissociation. To begin with, dissociation predicts susceptibility to cognitive failures, as indexed by Broadbent's Cognitive Failures Questionnaire (CFQ; Broadbent et al., 1982; Merckelbach et al., 2002). This self-report questionnaire measures the frequency of everyday lapses, such as forgetting names or being easily distracted unwillingly, features that have a raised prevalence in neurological disorders.

Secondly, high dissociators show disruptions in executive functioning (Cima et al., 2001; chapter 5) and a slowing of reaction times on the Stroop task (Freyd et al., 1998; chapter 4). These findings fit nicely with Klimesch et al.'s (1996, p. 511) words that " $\alpha$-frequency is significantly correlated with the speed of information processing."

Thirdly, various studies found dissociation to be related to commission errors (i.e., confabulations) in memory. Germane to this issue is the study described in chapter 2 , in which we found an association between heightened DES scores and memory commission errors. Furthermore, Candel et al. (2003) examined commission errors during free-recall in undergraduate students scoring high and low on the DES. They found that such commission errors were related to dissociation, with high DES scores going hand in hand with more commission errors. Clearly, the links between such memory errors and lowered $\alpha$ - and raised $\theta$-power require further study.

Our finding that dissociation was related to lowered $\alpha$-power might hint to a possible source of depersonalization and derealization phenomena. While the classical view is that $\alpha$-rhythms reflect cortical idling, more recent theories stress that the $\alpha$ rhythm is instrumental in increasing the signal-to-noise-ratio within the cortex by inhibiting conflicting processes to the task at hand (Cooper, Croft, Dominey, Burgess, $\&$ Gruzelier, 2003). Thus, it might well be the case that depersonalization experiences reflect a dysfunctional inhibitory process, leading to the influx of inappropriate information, thereby inducing depersonalization or derealization experiences. This interpretation is in line with a case report by Raimo, et al. (1999), who found reduced anterior and posterior mean $\alpha$-frequencies during alcohol-induced depersonalization (but see Simeon, 2004 for an overview of the neurobiology of depersonalization disorder).

Taken together, these findings suggest that the study of how cortical activity relates to dissociative experiences might be a fruitful research area. 
3 


\section{General Discussion}


The central theme of this dissertation concerned the defensive function, which is commonly ascribed to dissociative experiences in the clinical literature. In a number of studies, various predictions flowing from this dissociation-as-a-defense-mechanism view were empirically tested. Firstly, one would expect indiwiduals with heightened levels of dissociation to exhibit emotional blunting. Secondly, this view predicts that highly dissociative individuals will selectively deviate in their reaction to stimuli in one particular domain (to aversive stimuli), but not to other, for example neutral, stimuli. Thirdly, according to the defensive view, this reaction should manifest by influencing the processing of these aversive stimuli and/or omissions (i.e., dissociative amnesia). Furthermore, an alternative account of the origins of dissociative experiences was investigated. This alternative account states that dissociative experiences are due to a disrupted sleep-wake cycle. The following section provides an overview of the present findings.

\section{Summary of the results}

\section{Psychophysiological responsivity}

Three studies sought to investigate the connection between trait dissociation, as measured by the DES, and physiological responsivity as indexed by autonomic reactivity, such as the skin conductance or cortisol response. During a first study (chapter 2), participants were confronted with a highly emotional video fragment. In stark contrast to the supposedly defensive function of dissociation, higher levels of dissociation were accompanied by increased, not lowered, physiological responsivity during the video fragment. Likewise, Friedman's (2000) notion that dissociation might function as a compensatory mechanism to marked physiological arousal was not supported by our data.

Previous research in our lab (Smeets et al., 2004) showed that participants experience this particular video fragment, from the Hollywood movie American History $X$ (Kaye, 1998), as highly emotional. Still, one might object that such a movie fragment does not offer sufficient opportunity for personal involvement. This could, in turn lead to a more passive experience, thereby jeopardizing ecological validity. With this in mind, two additional studies (chapter 3) were conducted that relied on the Trier Social Stress Test (Kirschbaum et al., 1993). This task is known to reliably induce acute stress in the lab. Importantly, this task forces the participant to actively take part in the manipulation. That is, participants are undergoing a stressful job interview in combination with a difficult arithmetic task. In line with chapter 2 , these two additional studies failed to support the hypothesis that heightened levels of state dissociation lead to a reduction in physiological arousal during acute stress, as indexed by our physiological measures of acute stress (i.e., cortisol responses). As a matter of fact, dissociation was even related to heightened levels of subjective stress.

In sum, all three physiological studies failed to underpin the defensive function of dissociation, as indexed by physiological arousal during acute stress situations. In fact, tentative evidence evolved for a tendency of high dissociators to exhibit heightened arousal during acute stress.

General or specific deficit?

Besides emotionall blunting, the notion that dissociative symptoms are the manifestation of a defense mechanism presupposes that dissociation goes hand in hand with specific 
cognitive deviations to aversive stimuli, while no aberrations should occur in case of non-aversive information.

Chapter 4 describes a study which sought to investigate, whether dissociation has to be considered a selective attentional deficit (i.e., only in response to aversive stimuli) or is of a more global nature. To this end, a modified version of the Stroop color naming task (Stroop, 1935) was employed, consisting both of neutral and aversive stimulus words which are presented either during selective or divided attention condition. This study failed to detect any selective attentional deficits to aversive stimuli. Howewer, high dissociators were slower to react to all stimuli, irrespective of valence.

To replicate the finding of chapter 4 that dissociative experiences are related to global deficits in attentional functioning and to deepen our understanding of this relationship, the Random Number Generation Task was employed in chapter 5 . This was done so as to quantify subtle deviations in executive functioning. Of course, this task does not involve any emotional stimuli. Nevertheless, this study indicated that dissociation is related to subtle deficits in executive function. So, again we found evidence that dissociation is related to a global lack of attentional control.

Chapter 6 investigated the possibility that high dissociators might posses a cognitive advantage when suppressing aversive memories. These individuals might, for instance, posses a superior capability to dissociate (i.e., keep out of mind) aversive information. However, our findings from chapter 4 and 5 , showing that heightened levels of dissociation ane related to deficits in attentional control, suggest that the contrary might also be possible. A case in point is a study by Brewin and Beation (2002) showing that individuals' working memory capacity and fluid intelligence are predictive of their suppressive capabilities, while Engle (2002) postulates a strong bidirectional link between working memory capacity and attention. Contrary to the prediction that high dissociators might be able to dissociate the aversive information, a trend emerged with high dissociators being worse at suppressing the memory of the aversive video fragment, as indicated by a heightened frequency of intrusions.

Overall, the results from part II indicate that dissociative experiences are neither related to superior suppression of emotional material, nor to selective deviations in attention to aversive stimuli. What our findings do suggest, however, is that dissociation in undergraduate students manifests itself in global attentional deficits (i.c., independent of valence). Our findings do, however, deviate from findings by De Ruiter, Phal, Elzinga, and Van Dyck (2004), who found dissociation, as measured by the Dissociation Questionnaire (DIS-Q; Vanderlinden, Vandereycken, van Dyck, \& Vertommen, 1993), to be positively related to verbal working memory capacity. Interestingly and in contrast to De Ruiter et al. (2004), Rossini, Schwartz, and Braun (1996) found that a significant subset of his sample consisting of patients with Dissociative Identity Disorder $(26 \%$ ) exhibited subtle neuropsychological deficits on the Memoty/Distractibillity factor of the Wechsler Adult Intelligence Scalle. Moreover, we found no relation between dissociation, quantified by the DES and the Operation Span task (OSPAN; Tumer \& Engle, 1.989) in a sample of 113 (93 females) undergraduates (unpublished).

\section{Ammesia or confabulation?}

A related hypothesis concerns bigh dissociators' memory, as one of the core symptoms of dissociation is dissociative amnesia (American Psychiatric Association, 1994). Due to their (supposedly) exceptional ability to forget distressing events, high dissociators 
should exhibit more omission errors (i.e., less hits) for emotional stimull compared to control participants. However, in line with previous research (Brewin \& Saunders, 2001; Candel ef al., 2003; Kindt \& van den Hout, 2003), chapter 2 clearly indicates that high dissociators" memory for highly emotional stimulus material is far from impaired. These individuals do, as a matter of fact, report as many cortect details of an emotional video fragment as low dissociators. However, they show a distinct tendency to confabulate, i.e., make up information that was actually not present in the video fragment.

A related study ( $n=76$, unpublished) also made use of the same selective and divided attention condition as described in chapter 4 , while investigating the influence of dissociation on the performance in a Directed Forgetting (DF) paradigm. Thus it was investigated whether high dissociative participants possess superior capabilities to forget aversive information. Paralleling our study described in chapter 4, we employed both neutral and aversive stimulus words. In contrast to DePrince and Freyd (2004), but in line with McNally, Ristuccia, \& Perlman (2005), all participants exhibited better recall of emotional stimulus words irrespective of their frequency of dissociative experiences. Moreover, DF performance was unrelated to dissociation. These finding are difficult to reconcile with the defensive function attributed to dissociation.

\section{Sleep experiences and neurophysiology}

The fact that we failed to find any underpinning for the defensive properties of: dissociation in our studies highlights the need for altemative ideas about the origins of dissociative experiences. One alternative view was explored in part III of this dissertation. More specifically, we examined the notion that dissociative experiences might be fuelled by a labile sleep-wake cycle, which should lead to an effortless transition between sleeping and waking states. Therefore, in a first study (chapter 7) we tested, whether dissociative experiences were, indeed, related to various sleep experiences, such as narcolepsy, vivid and unusual dreams, and nocturnal experiences as waking dreams and sensing the presence of someone. While dissociative experiences were related to these sleep experiences, they were not linked to certain sleeping patterns (i.e., morningness vs. eveningness).

In chapter 1, we pointed out that individual differences in sleep experiences might account for the overlap between fantasy proneness and dissociation. This mediational influence was explored in chapter 8 . The study described in that chapter also evaluated the robustness of previous findings showing that dissociation and sleep experiences overlap. This mediational analysis, conducted along the lines of Baron and Kenny (1986), confirmed our hypothesis, as sleep experiences were found to account for a portion of the overlap between dissociation and fantasy proneness.

Carrying the chapter 7 and 8 line of reasoning one step further, the relationship between dissociation and sleep experiences was approached in another way in chapter 9 . Here, we strived to underpin the overlap between dissociation and sleep, while employing a measure that is independent of self-report. Therefore, resting EEG was measured as well as self-report measures of sleep experiences. While we did replicate the relationship between dissociation and self-reports of sleep experiences, no connection emerged between sleep experiences and the resting EEG. However, high dissociators appeared to exhibit distinct deviations in the resting EEG, which have been linked to hypnagogic states (i.e., the transition from the waking to the sleeping state) and deficits in both memory and attention (Klimesch, 1999). 


\section{Dissociation: A defensive maneuver?}

Based on our understanding of the literature, a number of predictions were deduced, which had to be fulfilled, if dissociative experiences were, indeed, the manifestation of a defense mechanism. All these predictions were empirically tested in one or more studies in our lab. In sum, the data from these various studies falled to fulfill each prediction flowing from the notion that dissociative experiences are the manifestation of a defense mechanism. More specifically, high dissociators did not show reduced arousal during acute stress. Our findings suggest that the opposite (i.e., dissociation is related to heightened arousal) seems to be even more probable. Secondly, these individuals exhibit no selective deviations in attention. Rather, their deficit seems to be of a more global nature. Thirdly, our study failed to detect any signs of amnesia, as index by recall performance. High dissociators memory even appeared to be over complete, in the sense that they exhibited a tendency to commit commission errors, i.e., to make up information that was not present in the stimulus materiall.

Germane to the issue that dissociation possesses defensive properties is a recent study in which we (Giesbrecht, Smeets, \& Merckellbach, submitted for publication) investigated an alternative way of inducing acute (i.e, peritraumatic) dissociation in a sample of undergraduates $(n=70)$. This was done by means of a cold pressor test (CPT; Mitchell, MacDonald, \& Brodie, 2004), which basically consists of asking the participants to hold their arm in ice water $\left(1-3^{\circ} \mathrm{C}\right)$ for as long as possible. The CPT led to a significant increase in acute dissociation, as measured by the revised version of the Peritraumatic Dissociative Experiences Questionnaire (PDEQ; Marshall, Orlando, Jaycox, Foy, \& Belzberg, 2002). This finding highlights the procedure's usefulness in inducting acute dissociative reactions. Another finding is worthy of note. Recall that the view that dissociative symptoms constitute the manifestation of a defense mechanism would predict that individuals with heightened levels of trait dissociation would make use of their habitual way of reacting to (even minor) stressors, which. should be acute dissociative symptoms (e.g., van der Kolk \& van der Hart, 1989). As during actual trauma, this use of dissociation as a coping mechanism should reduce suffering and consequently enable individuals that are high on dissociation to endure the task longer. Surprisingly, the increase in acute (i.e., peritraumatic) dissociative symptoms was unrelated to trait dissociation as measured by the DES and secondly and even more importantly, higher levels of trait dissociation were related to shorter times of sustaining the CPT. While being in concert with our previous findings (e.g., chapter $3,4)$, these linding are in stark contrast to the alleged defensive function of dissociation.

To conclude, the findings reported in this dissertation are impossible to reconcile with the view that dissociative experiences are the manifestation of a defensive mechanism. In addition, it is worthy of note that this view articulates why, but not how trauma produces dissociative symptoms, while being silent as to why certain benign personality traits, - notably fantasy proneness and cognitive failures -, overlap to a substantial degree with dissociative symptoms. That is, a number of studies (e.g., Merckelbach et al., 2005; Merckelbach et al., 1999; Rauschenberger \& Lynn, 1995) have found that people scoring high on dissociation also score high on measures of fantasy proneness (i.e., the tendency to engage in extensive and vivid fantasizing) and cognitive failures (i.e., proneness to everyday slips and lapses), with correlations being in the order of $.45-.70$. 


\section{Future perspectives}

\section{Sleep and the evolvement of dissociation}

Watson's (2001; 2003b) and our finding (chapter 7 to 9) that dissociative symptoms are linked to self-reports of unusual sleep-related experiences (e.g., flying dreams, hypnopompic imagery, sensing the presence of someone else) might offer a fresh. perspective on the etiology of dissociative experiences. In another related study, Rassin, Merckelbach, and Spaan (2001) showed that people who find it difficult to discriminate between vivid dreams and reality ("did it really happen or did I only drean?") have raised dissociation scores. Interestingly, McNally and Clancy (2005) recently noted that in their mixed sample of participants with memories of childhood sexual abuse, reports of sleep paralysis were typically found among those with raised dissociation scores. Likewise, Levin and Fireman (2002) and Agargun et al. (2003a) pointed out that there exists a substantial correlation between dissociative symptoms and nightmare frequency.

Recently, we used the Pittsburg Sleep Quality Index (PSQI; Buysse, Reynolds, Monk, Berman, \& et al., 1989) to study the sleep dissociation link in a large undergraduate sample $(n=306)$. The PSQI is a self-report measure of sleep quality and disturbances that consists of seven subscales: subjective sleep quality, sleep latency, sleep duration, sleep efficiency, sleep disturbances, use of sleep medication, and daytime sleepiness. Even in our very homogenous sample with no prominent sleep disorders, the PDEQ total and the DES score were modestly correlated $(r=.19)$. Interestingly, this relation was nearly solely carried by the daytime sleepiness factor which exhibited a correlation of $r=.28$ with the DES, while all other correlations between the DES and the other PSQI subscales were substantially smaller (all $r$ 's $<.18$ ).

On a related note, we (Giesbrecht \& Merckelbach, in press) investigated the possibility that the sleep dissociation link might be due to the positive response tendency that is associated with dissociative symptoms (e.g., Merckelbach \& Jelicic, 2004). That is why we administered the DES and the ISES together with the Structured Inventory of Malingered Symptomatology (SIMS; Smith \& Burger, 1997). The SIMS is a self-report measure that is designed to detect malingering of psychiatric disorders and cognitive impairments. This scale consists of items which describe bizarre experiences (e.g., "Sometimes my muscles go limp for no apparent reason so that my arms and legs feel as though they weigh a ton"), atypical symptoms (e.g." "At times, I am so depressed I welcome going to bed early to "sleep it off"), and approximate answers (e.g., "If you have US $\$ 1.50$ and I take fifty cents away, you will have 75 cents left"). As even psychiatric patients only seldomly endorse these items (Merckelbach \& Smith, 2003), in our study the SIMS was used as a measure of positive response tendency. In a sample of 87 undergraduates, dissociation was indeed accompanied by a positive response tendency, as indicated by a substantial correlation between the SIMS and the DES ( $r=$ .51). The relationship between the ISES and the DES, however, remained intact even when this positive response tendency was controlled for by means of a partial correlation $\left(r_{p a r f a l . S m S}=.35\right)$. Consequently, our data suggests that, while a positive response tendency was present in our sample, the relationship between dissociation and sleep experiences is not an artifact of this tendency.

In sum, all these findings clearly highlight the robustness of the relationship between sleep experiences and dissociation, both across different samples, but also across different measures of sleep experiences. Recently, Agargun, Kara, Ozer, Selvi, Kiran, and Ozer (2003b) demonstrated that the link between dissociation and sleep is not 
limited to healthy samples, but allso occurs in clinical samples, notably people with dissociative disorders.

Thus, there are good empirical reasons to assume that dissociative symptoms and deviant sleep phenomena are related to each other. One exciting interpretation of this link is as follows (see also Watson, 2001). For some, yet to be specified reason, some people have a labile sleep-wake cycle. This leads to intrusions of sleepphenomena (e.g., dreamlike experiences) into waking consciousness, which result in feelings of depersonalization and derealization. Apart from these intrusions of sleepphenomena, another well-documented consequence of disruptions of the sleep-wake cycle is its detrimental effect on memory (Hairston \& Knight, 2004) and attentional control (Jewett et al., 1999; Williamson et al., 2001). This might account for the general attentional deficits encountered in high dissociators (see chapter 4,5 ), i.e., cognitive failures. These orthogonal dimensions, consisting of intrusions of sleep-phenomena and deficiencies in memory and attention due to deviations in the sleep-wake cycle, together would then constitute the various dissociative symptoms.

This interpretation offers a fresh and exciting perspective on the origins of dissociation, because it may reconcile theories that previously seemed to be highly conflicting with one another. Thus, studies stressing that fantasy immersion and lack of cognitive control overlap with dissociative symptoms (Chapter 8; Merckelbach et al., 2002; Merckelbach et al., 2000a; Merckelbach et al., 1999) seem far away from studies assuming a traumatogenic etiology of these symptoms (Gershuny \& Thayer, 1999). Yet, both approaches can be integrated when it can be shown that disturbed sleep patterns are the final common pathway to dissociative symptoms. Indeed, although the details are far from clear, there is good evidence to show that patients suffering from Post Traumatic Stress Disorder (PTSD) exhibit an increase in nightmare frequency and REM sleep density, but also complain of insomnia. On the other hand, dissociative symptoms go hand in hand with raised frequencies of nightmare reports (Levin \& Fireman, 2002). Accordingly, the sleep-dissociation approach may explain how trauma (but not only trauma for that mattery causes dissociative symptoms and why dissociation, trauma, fantasy proneness, and cognitive failures overlap.

Another reason to be enthusiastic about the sleep-dissociation approach as described above is that it may ultimately offer an explanation as to why there is such a robust connection between dissociation and memory commission errors. That is to say, one of the most typical features of high dissociative people's cognitive architecture is that they tend to produce pseudo-memories (i.e., false alarms; commission errors) on memory tasks. This is true for undergraduates scoring high on dissociation (chapter 2; Candel et al., 2003; Merckelbach et al., 2000a), but it is also true for patients with PTSD (Bremner, Shobe, \& Kihlstrom, 2000). Currently, literature on dream and memory is burgeoning and one theory that deserves serious attention holds that the progression of waking state to REM is marked by an increase in "fluid" and hyperassociative thinking (Stickgold, Hobson, Fosse, \& Fosse, 2001). Thus, one could speculate that dreamlike intrusions into waking state that are typical for dissociation interfere with memory performance in such way that commission errors occur.

To be sure, so far, the idea that it is sleep abnormalities that produce dissociation et tutti quanti is a global idea. The sleep-dissociation approach is in need of further articulation. Thus, its attractiveness lies not so much in that it allows for very specific predictions, but rather in its potential to create order in what seems to be a chaotic pattern of findings. For example, there is a nontrivial, but poorly understood correlation between dissociation and schizotypy (Merckelbach, Rassin, \& Muris, 
$2000 \mathrm{c}$ ), while it is also know that schizotypy is a predictor of nightmare distress (Claridge, Clark, \& Davis, 1997). In a recent review (Giesbrecht \& Merckelbach, 2005), we explained how these apparently diverse phenomena may becone more understandable once one adopts the sleep-dissociation approach. So, the explanatory power of this approach is potentially substantial.

However, so far, findings documenting the relationship between dissociation and sleep rest on correlational data. One inherent limitation of this type of study is that it forbids the deduction of causal relations between various variables. Future studies should address this issue in two different ways, either by inducing sleep disturbance in healthy participants or by normalizing sleep in clinical groups.

Sleep disturbance can reliably be induced in healthy participants by depriving them of normal sleep. If dissociative experiences are, indeed, fuelled by a labile sleepwake cycle, one would expect that sleep loss would increase dissociative symptons, and memory commission errors (chapter 2), but will also decrease $\alpha$-power (chapter 9 ). Finding such pattern in sleep-deprived participants would provide support for the causal chain implied by the sleep-dissociation approach.

Sleep deprivation interferes with normal sleep patterns, but there are only few manipulations that may normalize sleep. However, a procedure called "simulated dawn" (Leppamaki, Meesters, Haukka, Lonnqvist, \& Partonen, 2003) has been proposed as a non-invasive technique to treat Seasonal Affective Disorders and one of its (claimed) effects is that it normalizes sleep-wake patterns. The technique employs a particular kind of light therapy that involves exposing participants each morning upon awakening to $30 \mathrm{~min}$ periods of $300 \mathrm{~lx}$. If this procedure should indeed lead to a reduction in sleep complaints and dissociation scores, then this would provide further evidence for the causal implications of the sleep-dissociation approach.

\section{The unity of dissociative experiences}

As is usual in the clinical literature, this dissertation has employed the concept of dissociation as shorthand for a variety of psychological phenomena. These range from common cognitive failures, such as lapses in attention, non-pathological absorption and dayclreaming to pathological dissociation in the strict sense, as represented by the dissociative disorders. However, even within these categories, the way in which dissociation manifests itself differs widely. Based on the phenomenological differences between dissociative amnesia, absorption, depersonalization, and derealization, one might speculate, that at least some of these different symptoms have nothing in common and rely on separate biological substrates. Accordingly, a number of authors have recently criticized the notion of a unitary dissociative process (Hacking, 1995; Holmes et al., 2005; Jureidini, 2003).

In line with Cardeña (1994) and Allen (2001), Holmes et al. (2005) recently contributed to this debate by proposing that one should distinguish between two distinct forms of dissociation, detachment and compartmentalization. The concept of detachment consists of symptoms of depersonalization, derealization, and related phenomena, such as out-of-body experiences. Psychopathological conditions that exhibit symptoms of detachment are depersonalization disorder and feelings of detachment during flashbacks in postraumatic stress disorder. The concept of compartmentalization, on the other hand, incorporates dissociative amnesia and somatoform dissociation, such as sensory loss and "unexplained" neurological symptoms (Nijenhuis, Spinhoven, van Dyck, van der Hart, \& Vanderlinden, 1998). The core feature of this concept is a deficit in deliberate control of processes or actions that 
would normally be amenable to this control, as can be found in Dissociative Identity Disorder or Somatization Disorder.

We are certain that our understanding of the underlying mechanism of dissociation might benefit greatly from the separate investigation of the various manifestations of dissociative experiences. At present, however, this possibility is limited by the availability of psychometric instruments, designed to differentiate between different types of dissociative experiences. In spite of its wide use in the literature, the DES appears to be of little use in this respect for a number of reasons. Firstly, this instrument has been designed to tap a unitary continuum of dissociative experiences (Bernstein \& Putnam, 1986). Nevertheless, various authors argued that the DES should be vilewed as consisting of more than one dimension (Carlson et al., 1991; Ross et al., 1995). However, Waller (1995) ascribed these findings to the widely differing item endorsement rates of the DES. Notably, his reanalysis of the Carlson et al. (1991) data yielded only one single factor. In line with this, our own (unpublished) factor analysis of the Dutch translation of the DES in a sample of 940 undergraduate students underpinned this single factor solution. Based on these findings, these subscales do not offer a means to study different types of dissociative symptoms separately.Another seemingly promising approach is the DES-T (Waller et al., 1996). This scale was derived via a taxonometric method and was designed as a measure of pathological dissociation. This scale only includes DES items that do not tap fantasy immersion and absorption, but pathological manifestations of dissociation, such as depersonalization, derealization, and amnesia. The DES-T has been widely used in the clinical literature (Irwin, 1999; Simeon, Guralnik, Schmeidler, Sirof, \& Knutelska, 2001; Tampke \& Irwin, 1999; Waldo \& Merritt, 2000; Waller, Ohanian, Meyer, Everill, \& Rouse, 2001). Simeon et al."'s (1998) finding that the DES-T total score performs better in differentiating patients with depersonalization disorder from healthy controls than the standard DES total score underpins the validity of the DES-T. The temporal stability of the dissociative taxon membership probability was investigated by Watson (2003a) in a sample of 465 undergraduates. His study yielded a disappointing test-retest correlation of 34 of the taxon membership probability. Our own study employing the DES-T taxon membership probability yilelded a somewhat higher, but still unsatisfactory test-retest correlation $(r=.44$, chapter 6 , table 6.2). Due to this result, Watson cautions researchers to employ the taxon probability as a measure of pathological dissociation in non-clinical samples (see, for a similar finding, Modestin \& Emi, 2004). In addition, Watson (2003a) reports a test-retest correlation of .62 for the DES-T total score after 2 month, which is substantially lower than the stability of the DES total score. Our own research (chapter 6) shows that Watson's study might have underestimated the stability of the DES-T total score, as after an interval of four months the test-retest correlation of the DES-T total score $(r=.82)$ did not differ from the DES s stability $(r=.84)$. However, the main objective during the development of the

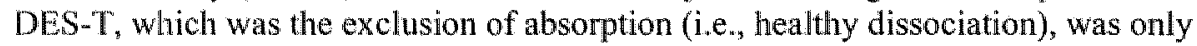
partially successful. As a matter of fact, the overlap between dissociation, as indexed by the DES-T and lantasy immersion is, indeed, reduced, but remains robust (Levin \& Spei, 2003). Our own study, however, indicates that employing the DES-T results in virtually no reduction in associative strength between dissociation and fantasy proneness (chapter 8). While the DES-T and especially its taxon membership probability might lack the sensitivity to serve as a diagnostic instrument for dissociative disorders (Simeon, Knutelska, Nelson, Guralnik, \& Schmeidler, 2003), its total score can be useful, as it does focuses on the less common and probably the more pathological 
experiences of dissociation. This is illustrated by our studies in which the DES-T was related to the frequency of thought intrusion (chapter 6) and minor executive dysfunctions (chapter 5 ), while the DES total score was not. However, in other studies the DES-T did not lead to fundamentally different findings (e.g., chapter 7 ), which is also reflected by correlations between the DES and the DES-T total score which range from .84 to .89 in our own studies.

Two other instruments that might prove fruitful in separating different dissociative symptoms, are the Depersonalization Severity Scale (DSS; Simeon, Guralnik, \& Schmeidler, 2001) and the Somatoform Dissociation Questionnaire (SDQ20; Nijenhuis, Spinhoven, Van Dyck, Van der Hart, \& Vanderlinden, 1996). The DSS is a clinician-rated scale that assesses the severity of symptoms of depersonalization and could be employed as a measure of detachment. This instrument possesses high convergent validity and proved to be sensitive to treatment changes in patients with depersonalization disorder. The SDQ-20 is self-report instrument that quantifies somatoform dissociation, such as analgesia, anesthesia, and loss of consciousness. While Holmes et al. (2005) incorporated somatoform dissociation in their concept of compartmentalization, the DES does not include these symptoms, which makes the SDQ-20 a worthwhile addition to the DES. However, the discriminant validity of these two scales with respect to fantasy immersion and the susceptibility to cognitive failures is unknown and has to be investigated. Moreover, altemative measures that quantify distinct types of dissociative symptoms separately still have to be developed.

\section{Inducing dissociative experiences}

Above, we briefly discussed two different ways in which dissociative symptoms might be enhanced or reduced. These methods rely on manipulation of sleep patterns, but have not been investigated, yet. However, there are other manipulations that are known to reliably induce dissociative experiences. For example, states of sensory deprivation have been shown to elicit dissociative symptoms (Leonard, Telch, \& Harrington, 1999) and out-of-body-experiences (McCreery \& Claridge, 1996). Based on the notion that dissociative experiences are fuelled by a disturbed sleep-wake cycle, one would expect sleep deprivation to act as a catalyst leading to even more frequent and severe dissociative episodes during such experimental procedures.

In addition, it is known that certain drugs, notably low doses of the anestheticum ketamine, produce dream like states and dissociative symptoms, e.g., participants in Krystal et al.'s (1994) studly reported slowing of time and alterations in the vividness, form, and context of sensory experiences following the administration of keramine. It is hypothesized that these dissociative states are due to diminished NMDArelated neurotransmission (Simeon, 2004). Interestingly, the dissociation inducing properties of cannabinoids (Simeon, Knutelska, Nelson, \& Guralnik, 2003), such as marijuana, might also be mediated via NMDA receptors. Another class of chemicals does elicit dissociation and more specifically depersonalization reactions in healthy participants. This class is the hallucinogens, such as lysergide (LSD). As these chemicals act as agonists of serotonin $5-\mathrm{HT}_{2 A}$ and $5-\mathrm{HT}_{2 \mathrm{C}}$ receptors, a possible mediational role for serotonin in dissociation is implied (Simeon, 2004). In sum, these findings might hint at the neurobiological basis of depersonalization experiences.

Furthermore, future research might be directed at the induction of the separate manifestations of dissociation, e.g., depersonalization, absorption, and amnesia. One might expect, for instance, that some manipulations, which are associated with extremely low levels of arousal, such as sensory deprivation, would more readily elicit 
dissociative experiences like absorption. Manipulations, however, which tend to lead to extreme levels of arousal, such as skydiving (Sterlini \& Bryant, 2002), might be more strongly related to feelings of derealization and time distortion.

\section{Psychophysiology of dissociative experiencers}

As pointed out in chapter 2 and 9 , the psychophysiology of dissociative experiences is an under-researched domain (Scaer, 2001). This is astonishing, as hyperresponsivity in Posttraumatic Stress Disorder, for instance, is extensively studied (e.g., Bryant, Harvey, Gordon, \& Barry, 1995; Wolfe et al., 2000). In contrast, little is known about emotional blunting in high dissociators and the dissociative disorders. The few studies that have been conducted yielded mixed findings. For example, two studies investigated how peri-traumatic dissociation during actual trauma relates to subsequent physiological reactions to trauma narratives. While Griffin et all. (1997) reported reduced physiological arousal in high dissociators, Nixon, Bryant, Moulds, Felmingham, and Mastrodomenico's (2005) found a trend towards heightened responsivity (see, for simillar results, Ladwig et al., 2002). Our own results (see part 1) are in line with the latter finding, as we too failed to find any evidence for emotional blunting. In addition, our studies yielded tentative evidence for hyperresponsivity in high dissociators. Morevoer, limited evidence is available, hinting that patients with depersonalization disorder demonstrate selective inhibition of emotional processing (Sierra et al., 2002). In sum, a systematic analysis of how dissociation is implicated in the regulation of arousal would greatly contribute to our understanding of dissociation.

In the long run, research might even succeed in deducing objective psychophyiological markers for the different clusters of dissociative symptoms (i.e., detachment and compartmentalization). These markers could be either episode markers that are only present during an episode of a disorder (i.e., during a particular acute dissociative symptom) or vulnerability makers, which are traits that are present before, during, and after an acute episode (lacono \& Ficken, 1989). These markers discriminate individuals who are at risk of developing a particular disorder from others who are not. The discovery of either episode or vulnerability markers would enable the quantification of dissociative symptoms and specifically the diagnosis of dissociative disorders, independent of self-report. A vulnerability marker could, for example, consist of the responsivity of the autonomic system to aversive stimuli (e.g.s acoustic startle). Interestingly, Guthrie and Bryant (2005) report in a recent article that an increased startle response prior to trauma exposure might be a vulnerability factor for the subsequent development of posttraumatic stress disorder. A possible episode marker might, on the other hand, consist of a distinctive neurophysiological pattern of activity which might be intimately related to a particular symptom of dissociation. In keeping with this, we showed that heightened levels of dissociation are related to a particular pattern of neurophysiological activity (see chapter 9). While this is a necessary step, this is far from sufficient. To function as an effective objective marker, it has to be demonstrated that this activity cannot be elicited by procedures that are aimed at replicating the arousal level of dissociation, as it cannot be precluded that, e.g., relaxation might result in a comparable neurophysiological pattern as absorption. Moreover, it has to be investigated whether that the pattern of activity cannot be induced by suggestion (Jureidini, 2003). In addition, one has to assure that other factors, such as fantasy proneness or schizotypy, do not better account for this pattern of activity. Obviously, there is potentially much to be gained from a thorough investigation of the psychophysiology of dissociation. 


\section{Final remarks}

To conclude, the data that we accumulated in our multiple samples of undergraduate students are definitively at odds with the notion that dissociative experiences might represent the manifestation of a defense mechanism. Yet, our findings need to be replicated in clinical samples consisting of patients with severe dissociative pathology, such as depersonalization or a diagnosis of dissociative identity disorder. Nevertheless, based on our own findings and our reading of literature, we are tempted to dismiss the notion that dissociative experiences possess defensive properties.

In sum, the study of dissociation is in need of aiternative approaches in both theoretical as well as empirical respect. Our understanding of dissociation might, for instance, gain much from the study of sleep and dissociation and its explanatory potential explained above. Another important step, which some clinicians may consider drastic, might prove necessary. This is the abolishment of the idea that there is one unitary dissociative process, as this idea might impede our understanding of the (probably multiple) underlying mechanisms of dissociative experiences. In addition, research on dissociation should to a much greater extent rely on objective psychophysiological measures. The necessity of this point is illustrated by the mixed findings concerning emotional blunting, which is regularly considered being a core feature of high dissociators responses to aversive stimuli, but is still not convincingly demonstrated.

Meanwhile, practitioners and scientists should be aware that while "there is no good evidence for a traumatic etiology of DID or any other dissociative disorder" (Kihlstrom, 2005, p. 14) and the defensive properties of dissociation cannot be convincingly demonstrated, dissociative experiences should not be interpreted as being the manifestation of a defense mechanism. 


\section{References}

Agargun, M. Y. Kara, H., Ozer, O. A., Selu, Y., Kiran, U., Kuran, S. (2003a). Nightmares and dissociative experiences: "The key role of childhood tramatic events. Etwopedn Archives of $P$ sychotry and Chincal Newosclences, 57, 139-145.

Agargun, M. Y. Kata, H, Oze, O. A., Selvi, Y., Kiran, U., Ozer, B. (2003b). Clinical importance of nightmare disorder in patients with dissociative disorders. Europecon Archives of Psychiany and Chincal Neuroscience, 57, 575.579.

Allen, J. G. (2001). Thaumaric relationships and serious mental disorders. New York, NY: lohn Wiley and Sons.

Allen, J. J., Coutri, J. A., \& Nazarian, M. (2004). Ussues and assumptions on the road from taw signals to metries of frontat EQC asymmetry in emotion. Biological Psychology, 67, 183-218.

American Psychatric Association. (1980). Diagnostic and statistical manual of menat disorders (3rat ed). Washington, D.C.:A.A.

American Psychiatric Association. (1994). Diagnostic and statistical mannal of mertal disorders (fih. ed). Washington, D.C: APA.

Arnstronge, J. G., Putnam, F. W., Carlson, E. B., Libero, D. Z, \& Smith, S. R. (1997). Development and validation of a measuite of adolescent dissociation: The Adolescent Dissociative Experience Scale. Jounat of Nervows and Menual Disease. 185, 491-497.

Buron, R., \& Kenny, D. (1986). The moderator-mediator variable distinction in social psychological research: Conceptual, strate gic, and statistical considerations. Journal of Personalioy and Social Psychology, $51,1173-1182$.

Beaty, J., Greenberg, A., Deibler, W. P., \& OHanlon, J. F. (1974). Operant control of occipital theta rhytm attects performance in a radar monitoring task. Sclence, $183,871.873$.

Becker-Blease, K. A. Deater-Deckard, K., Eley, T., Fucyd, J. J., Stevenson, J. \& Plomin, R. (2004). A genetic analysis of individual differenees in dissoeiative behaviors in childhood and adolescence. Journat of Child Psychology and Psychiatry and Alied Disciplines: 45, 522-532.

Bergeman, C. S., Plomin, R., Pedersen, N. L., McClearn, G. E. Nesselroad, J. R., Costa, P. T„, et al. (1993). Genetic and enwironmental effects on openness to experience, agreeableness, and conscientiousness. Journal of Personality, 67, 159-179.

Bernstein, E. M., Putnan, F. W. (1986), Developnent, rellability, and validity of a dissociation scale. Nownal of Nerwous and Mental Disease, 174, 727-735.

Besthorn, C., Zertuss, R., Geiger-Kabisch, C., Sattel, H., Daniel, S., Schreiter-Gasser, U., et al. (1997). Discrimination of Alzheimer's disease and nomal aging by EEG data. Electroencepholography and Clinical Neurophysiology, 103, 241-248.

Blagrove, M. (1996). Effects of length of sleep deprivation on interrogative suggestibility. Jouphal of Experiwnenal Psychology: Applied, 2, 48-59.

Biagrove, M., Hartnell, S. J. (2000). Lucid draning: Associations with internal locus of control, need for cognition and ereativity. Perwowality and mavidual Diferences, $28,411.47$.

Boonwsma, D. 1. (1998). Genetic analysis of Cognitiwe Faihures (CFO): A study of Dutch adolescent twins and the it parents. European Journol of Personality. 12,321-330.

Bremner, J. D., Shobe, K. K., Ke Kihlstrom, J. F. (2000). False meniories in women with self-reported childhood sexulat abuse: An empirical study. P5ychological Science, $11,333-337$.

Brewirs, C. B.. Beaton, A. (2002). Thought suppression, intelligence, and working memory capacity. Behowiow Research and Therapy, $40,923-930$.

Brewin, C. R., \& Saunders, I. (2001). The effect of dissociation at encoding on intrusive memories for a stressful lilm. British Joumal of Medical Psycholog, 74.467-472.

Bingathent, D. E., Cooper, P. F. Fitzgerald, P., \& Pakes, K. R. (1982), The cognitive fuilures questionnaire (CFQ) and its conelates British Jownal of Clinical Psychology. 21, 1-16.

Brunner, R.. Parzer, P., Sehuld, V. \& Resch, F. (2000). Dissociative symptomatology and traumatogenic factors in adolescent psychiatric patients. Jonrnol of Nervous and Mental Disecase, 188, 71-77.

Bryant. R. A. Harvey, A. G. Gordon, E. \& Barry, R. J. (1995). Gye movement and electrodermal responses to threat stimuli in postotraumatic stress disorder. Mnemaional Jownal of Psychophysiology, 20 , $209-213$

Buysse. D. J., Reynolds, C. F. Monk, T. H., Berman, S. R., \& et al. (1989). The Pittsburgh Sleep Quality Index: A new instrumeni for psychiatric practice and research. Psychiatry Research, 28, 193-213.

Canclel, 1., Merckelbact, H. (2003). Fantasy proneness and thought suppression as predictors of the Medical Student Syndrome. Personality and Indinidual Differences, 35, 519-524.

Candel, L. \& Merckelbach, H. (2004), Peritraumatic dissociation as a predictor of PTSD: A critical review. Comprenensine Pspchiany, 45, 44-50. 
Candel, L., Merckelbach, H. \& Kuijpers, M. (2003). Dissociative experiences are retated to conmussions in emotional memory. Behaviou Research and Therayy, 41,719-725.

Cardefia, E. (1994). The domain of dissociation. In S. J. Lymn \& J. Rue (Eds.), Dissoctation: Chinhal and theoretical perspectives (pp. 15-31). New York: The Guilford Press.

Carlson, E. B., Putnam, F. W. Ross, C. A., Anderson, G., Clark, P., Torem, M., at al. (1991), Fuctor analysis of the Dissociative Experiences Scale: A multicenter study. In B. G. Braun \& E. B. Cartson (Eds.),

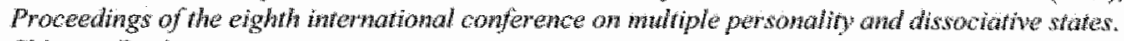
Chicago: Rusiti.

Chodoff, P. (1974). The diagnosis of hysteria: An overview. Anerican Joumal of Psychiany, 131. 1073-1078.

Cima, M., Merckelbuch, H. Hollnack, S., Butt, C., Kremer, K., Schellbach Matties, R., et al. (2003). The other side of malingering: Supernormality. Chinical Newropsychologigit, 17, 235-243.

Cima, M., Merckelbach, H., Klein, B. Schellbach-Matties, R., \& Kremer, K. (2001). Frontal lobe dysfunctions, dissociation, and trama self-reports in forensic psychiatric pattents, sourmal of Nervous and Mental Disease. 189, I8 88-190.

Claridge, G., Clark, K. \& Davis, C. (1997). Nightmares, dreans, and schizotypy, Britsish Joumol of Chimical Psycholog: 36, 377-386.

Classen, C., Koopman, C., \& Spiegel, D. (1993). Trauma and dissociation. Bulletin of Whe Menninger Clinic. $57,178-194$

Cooper, N. R., Croft, R. J., Dominey, S. J., Burgess, A. P., \& Giruzelier, J. H. (2003). Paradox lost? Exploring the role of alpha oscillations during externally vs. intemally directed attention and the implications for idling and inhibition hypotheses. Inemational Journat of Psychophysiology, $47,65-74$.

Council, J. R. (1993). Context effects in personality research. Curren Direchons in Psychological Science, 2 , $31-34$.

Council. J. R., \& Kirsch, 1. (1996). Explaining context effects: Expectancy and consistency. Contemporary Hypnosis, 13, 29-32.

Crick, F. \& Mitchison, G. (1995). RleM sleep and neural trets. Behowioural Broin Research, 69, 147-155.

Dalenberg, C. J., \& Palesh, O. G. (2004). Relationship between child abuse history, tratma, and dissociation in Russian college students. Chidd Abuse and Negled, 28, 461-474.

De Ruiter, M. B., Phat, H. R., Elizinga, B. M., \& Van Dyck, R. (2004). Dissociative style and individual differences in verbal working memory span. Consctowshess and Cogwinon, 13,821-828.

Dement, W., Kleitman, N. (1957), Cyclis variations in EEG during sleep and their relation to eye movennents, body motility, and dreaming. Electroencephalogrophy and Clinical Neurophosiology. $9,673-690$.

DePrince, A. P., \& Freyd, J. J. (1999). Dissociative tendencies, altention, and memory. Psychological Science. $10,449-452$.

DePrince, A. P., \& Freyd, J. J. (2004), Forgetting trauma stimuli, Psychological Science, 15, 488-492.

Dickerson, S. S., \& Kemery, M. E. (2004). Acute stressors and cortisol responses: A theoretical integration and synthesis of laboratory research. Psychological Bulletin, 130,355-391.

Doraby, M. J. (2001). Dissociative identify disorder and memory dysfunction: The current state of experimental research and its future directions. Clinical Psychology Review, 2l, 771-79s.

Dow, R. (1997). Psylab (Version 7). London: Contact Precision linstruments..

Whilers, A., \& Clark. D. M. (2000). A cognitive model of postraumatic stress disorder. Behowlou Research and Therapy, 38, 319-345.

Eisen, M. L. \& Carlson, E. B. (1998). Individual differences in suggestibility: Examining the infuence of dissociation, absorption, and a history of childhood abuse. Apphed Cognithe Psychology, $12, \$ 47 \%$ S61.

Engle, R. W. (2002). Working memory capacity as execulive attention. Current Dircetions in Pswehotogical Science. $11,19-23$.

Experimental Timing Standards Laboratory. (2001). Vis Sim-Resp: Voice Key. Retrieved 25.9.2007, Irom htip:/ciwebctyork.ac.uk/ETSL/vis_stim-resp_wotce_key.htm!

Fischer, D. G.. \& Elnitsky, S. (1990). A factor analytic sidy of wwo scalles measuring dissociation. Amerkan Jownal of Clinical Hypnosis, 32, 201-207.

Fowles, D. C. (1980). The three arousal model: Implications of Gray"s two-factor learning theory for heart rate, electrodermal activity, and psychopathy. Psychophysiology: $77,87-104$.

Frazier, P. A., Tix, A. P. E Banon, K. E. (2004). Testing moderator and mediator effects in counseling psychology research. Jownal of Counseling Psychalogy, $\$ 1,115-134$.

Freud, S. (1907/1906). Delusions and dreams in Jensen 's Growiva Srandard Edition. London: Hogarth Press.

Freyd, J. J., Martorellio, S. R., A.lvardo, J. S. Hayes, A. E. \& Christman, J. C. (1998). Cognitive envilonments and dissociative tendencles: Performance on the standard Stroop task for high versus low dissociators. Applied Cogntive Psychology, 12, \$91-\$103. 
Friedman, $M$ (2009). What might psychobiology of PTSD teach us about fiture approaches to pharmacolnerapy? Jow of Climieat Psychatry, 61,44-51.

Gardner Medwin, A. R. \&aul, S. (1995). Possible medhanisms for reducing memory confusion during steep. Behavioural Brain Resetwich, 69, 167-175.

Gass, U., Rodewald, f., Nickel, V., \& Emrich, H. M. (2001). Brevalence of dissociative disotders among psychiatrie inpatients in a german university clinic. Journal of Nerwous and Menfal Disease. 189 , $249-257$

Gershuny, B. S., \& Thayer, J.F. (1999). Relations among psychological trauma, dissociative phenomena, and trama-related distress: A review and integration. Chincal Psychology Review, 19, 631-637.

Gianoti, F., Cortessi, F., Sebastiani, T. \& Ottaviani, S. (2002). Circadian preference, sleep and dentime behavoutr in adoleseence. Journat of Sleep Research. 11. 191-199.

Giesbrecht, T. \& Merckelbach, H. (2004). Subjective sleep experiences are related to dissociation. Personality and lindividual Differences, 37,1341-1345.

Giesbrecht, T., \& Mercketbach, H. (2005), Obor dic kausale Beziehung zwischen Dissoziation und Tratuma: Ein kritischer Uberblick. Nenvenorzt, 76, 20-27.

Giesbrecht, T., \& Merckelbach, H. (in press). Dissociatieve Symplomen en Slaap [Dissociative symptoms and sieep]. Tijdscheift wor Psychatrie [Jowenal of Psychiatry].

Giesbrecht, T. Merckelbach, H., Geraerts, E., Smets, E. (2004). Disruptions in executive functioning and dissociation in undergruduate students. Jouphal of Nervous and Mental Disease, 192, 567-569.

Giesbrecht, T., Smeets, T. \& Merckelbach, H. (submitted for publication). Dissociative experiences on ice Peritraumatic and trat dissociation during the cold pressor test.

Gimsburg, N., \& Karpiuk, P. (1994). Random generation: Analysis of the responses. Perceprual and Moror Skills. 79. 1059-1067.

Gtiffin, M. G., Resick. P. A. Mechanic, M. B. (1997). Objective assessment of peritratmatic dissociation: Psychophysiological indicators. Anerican Jonnal of Psychiary, 154, 1081-1088.

Gudjongsson, G. H. (1984). A mew scale of interrogative suggestibility. Personality and lndwiduch Differences, S. $303-314$.

Guthrie, R. M., \& Bryant, R. A. (2005). Ataditory startle response in firefighters before and after trauma exposure. American Joumal of Pychatry, 162, 283-290.

Hacking, 1. (1995). Rewriting the sout: Multiple persondity and the sciences. Princeton: Princeton University Press.

Hagemann. D. (2004). Individual differences in anterior EEG asymmetry: Methodological problems and solutions. Biological Psychology, 67, 157-182.

Hairston, I. S., \& Knight, R. T. (2004). Neurobiology: Sleep on it. Nature, 430, $27-28$.

Harvey, A. G., Jones, C., \& Schmidt, D. A. (2003). Sleep and postraumatic stress disorder: A review. Clinical Psychology Review, 23, 377-407.

Hoeping, W., \& de Jong-Meyer, R. (2003). Differentiating unwanted intrusive thoughts from thought suppression: What does the White Bear Suppression Irvventory measure? Personality and Individual Differences, 34, $1049-1055$.

Holmes, E. A., Brewin, C. R. \& Hennessy, R. G. (2004). Trauma films, information processing, and intrusive memory development. Jow whal Experimental Psychology: General. 133, 3-22.

Holmes, E. A. Brown. R. J., Mansell, W., Fearon, R., Hunter, E. C. M. Frasquilho, F., et al. (2005). Are there two gualitatively distinct forms of dissociation? A review and some clinical implications. Chincal Psylokgy Review. 25, 1-23.

Holtgraves, T., \& Stockdale, G. (1997). The assessment of dissociative experiences in a non-elinical population: Reliability, walidity, and factor structure of the Dissociative Experiences Scale. Personality and hadividual Dofferences, 22,699-706.

Home, J. A. \& Ostberg, O. (1976). A self-assessment questionnaire to determine momingmess-eveningness in

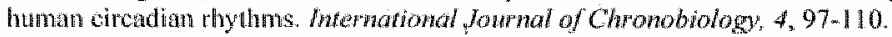

Horne, R. L.. Evans, F. J., \& Orne, M. T. (1982), Random number genetation, psychopathology and thertapeutic change. Archives of General Psychiatry. 39, 680-683.

Hyman, I. Wi, Jr., \& Billings, F. J. (1998). Individual differences and the creation of false chiddrood memories. Memory 6, $1-20$.

Hcono, W. G. \& Fickien, J. W. (1989). Research strategies employing psychysiological measures: ldentifying and using psychophysiological matkers, In $\mathrm{O}$. Turpin (Ed.), Handbook of clinical psychophwsiology (pp. 45-70). Chichester: New York: Wiley.

Irvin. H. J. (1998). Dissociative tendencies and the silting duck: Are self-reports of dissociation and victimization symptomatio of neviroticism? Journal of Chinical Psychology, 54, 1005-1015.

Irwin, H. I. (1999). Pathological and nonpathological dissociation: The relevance of childhood trauma. Journal of Pswchology: Interdisciplinasy and Applied, 133, 157-164. 


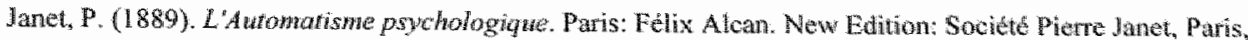
1973 .

Janet, P. (1894). LAunonatisme psychologique. Paris: Societe Pierte Jand.

Jansen, D. M., \& Frijda, N. H. (1994). Modulation of acoustic startle response by film-induced fear and sexual arousal. Psychophysiology: 31,565-571.

Jewett, M. E., Dijk, D.-J., Kronauer, R. E., Dinges, D. F. (1999). Dose-response relationship between steep duration and humar psychomotor vighlance and subjective aletthess. Sleep. 22, 171.179.

Johnson, R. C., Edman, J. L., D Danko, G. P. (1995). Self teported negative experiences and dissociation. Personality and Individual Differences, $18,793-795$.

Jureidini, J. (2003). Does dissociation offer a useful explanation for psychopathology? Psychopazhologa. 37. $259-265$.

Kaye, T. (1998). American History X. Los Angeles: New Line Cinema.

Kersting, A., Reutemann, M., Gast, U., Ohrmann, P.s Suslow, T., Michael, N., et al. (2003). Dissociative disorders and traumatic childhood experiences in transsexuals. Jowral of Nerwous and Mewal Disease, 191, 182-189.

Kihlstron, J. F. (1994). One hundred years of hy steria. In S. J. Lymn \& J. Rhue (Eds.), Dissociarion: Chinical and theorerical perspective (pp. 363-394). New York: Guildtord Press.

Kihlstrom, J. F. (2005). Dissociative Disorders. Amual Keview of Chincal Pspcholowy, 1, 1-27.

Kind, M., \& van den Hout, M. (2003). Dissociation and menory tragmentation: Experimental effects on neta-menory but not on actual menory performance. Behoviou Research and therapy, 4/, 167178.

Kirschbaum, C., \& Hellhammer, D. H. (1994). Salivary cortisol in psychoneuroendocrine research: Recent developments and applications. Psychonearoendocrinology, $19,3.134333$.

Kirschbaum, C.. Pirke, K. M. \& Hellhammer, D. H. (1993). The Trier Social Stress Test': A tool for investigating psychobiological stress responses in aboratory setting. Neuropsychobology. 28 , $76-81$.

Klimesch, W. (1996). Memory processes, brain oscillations and EEG synchronization. Jwreswational Journal of Psychophysiology, 24,61-100.

Klimesch, W. (1999). EEG alpha and theta oscillations reflect cognitive and memory perfomanee: A review and analysis. Brain Research Reviews, 29, 169-195.

Klimesch, W., Doppelmayr. M., Schimke, H., R Pachinger, T. (1996). Alpha frequency, reaction time, and the speed of processing information. Joumal of Climical Neurophysiology, $13,511-518$.

Klimesch, W., Vogt, F, \& Doppelmayr, M. (1999). Interindividtal diflerences in alpha and theta power reflect memory performance. Intelligence, $27,347-362$.

K.limesch, W., Vogt, F. \& Doppelmayr, M. (2000). Interindiwidual differences in alpha and theta power reflect memory performance. Intelligence. $27,347-362$.

Koopman, C., Carrison, V., Butler, L. D., Sudhakar, S., Palmer, L., \& Steiner, H. (2004). Relationships of dissociation and childhood abuse and neglect with heart rate in delinguent adolescents. Jowrnal of Traumatic Stress, 17, 47-54.

Kring, A. M., \& Gordon, A. H. (1998). Sex differences in emotion: Expression, experience, and physiology. Jounal of Perconality and Sucial Psychology, 74, 686-703.

Krystal, J. H., Karper, L. P., Seibyl, J. P., Freeman, G. K., Delancy, R., Bremner, I. D., at all. (1994). Subanesthetic effects of the noncompetitive NMDA antagonist, ketamine, in humans. Psychotomimetic, perceptual, cognitive, and neuroendocrine responses. Archives of General Psychiatioy, 51, 199-214.

Ladwig. K. H., Marten-Mittag, B., Deisenhofer, I., Hothann, B., Schapperer, J., Weyerbrock, S., et al. (2002) Psychophysiological correlates of peritrunnatic dissociative responses in survivors of lifetheatening cardiac events. Psychopathology, 35, 241-248.

Lang, K. L., Paris, J. Zweig.Frank, H., \& Liwesley, W. 1. (1998), Twin study of dissociative experiences. Jourpal of Abnomal Psychology, 186, 345-351.

Leonard, K. N.. Telch, M. J., \& Harrington, P. J. (1999). Dissociation in the laboratory: A comparison of strategies. Behuniour Research and Theraps. 37, 49-61.

Leppanaki, S.. Meesters. Y., Haukka, J., Lonnqvist, J., Le Partonen, T, (2003). Effect of simulated dawn on quality of sleep-a community-based trial. BMC Psycharry, 3,14.

Levin, R., Fireman, G. (2002). Nighimare prevalence, nightmare distress, and self-reported psychological disturbance. Sleep, $25,205-212$.

Levin, R, \& Spei,E (2003). Relationship of purported weasures of pathological and nompathological dissociation to self-reported psychological distress and fantasy immersion. Assessment, 1,160 168.

Lezak, M. D. (2004). Profile of Mood States (POMS). In M. D. Lezak, D. B. Howieson \& D. W. Loring (Eds.), Neuropsychological assessmenr (pp. 786-787). Oxford: Oxford University Press. 
Leak, M. D. Howieson, D. B., Loring, D. W. (2004) Neuropsychological Assessment. Oxford: Oxford University Press.

Lundgren, d. Berggren, U., \& Cafsson, \$. G. (2004). Psychophysiological reacions in dental phobic patients with direct wis. indired fear acquisition. Jownal of Behaviom Therapy and Experimental Psychiatry. $35,3-12$.

Macte, J. Cicchent, D., \& Toth, S. L. (200 \% The development of dissociation in maltreated preschool-aged children. Dew lopmen and Psychopathology. 13,233-254.

Markowitsch, H. I. (2003). Psychogenic annesia. Newromage, 20, S132-138.

Marshall, G. N., Orlando, M, Jayeox, L. H. Foy, D. W., Belzberg, H. (2002). Development and validation of a modified version of the Peritratmatic Dissociative Experiences Questionaire. Psychological Assersiment; $14,123 \times 134$.

Martinez Taboas, A, \& Bernal, G. (2000). Disscciation, psychopathology, and abusive experiences in a nonelinical Lutino university student group. Cu/nowd Diversity and Ethmic Minority Pspchology. 6, $32-41$.

MoCreery, C. \& Claridge, G. (1996). A study of hallueination in normal subjects-l. Self-report data. Persanalio and Individual Differewess, $21,739-747$

MeNain, D. M.; Lort, M. \& Droppleman, L. F. (1992). The Prafile of Mood States (POMS) Manual. San Diego, CA: EdTS.

McNally, R. J., de Clancy, S. A. (2005). Sleep paralysis in adults reporting repressed, recovered, or continuous memories of childhood sexual abuse. Jound of Anwety Disorders, 19,595-602.

McNally, R. J., Ristuceia, C. S. \& Periman, C. A. (2005). Forgetting of trauma cues in adtulls reporting continulous or rocovered memories of abuse. Psychological Science, 16, 336-340.

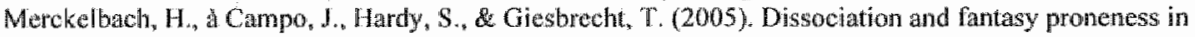
psychiatric patients: A prelininary study. Compreherisive Psychiatry. 16, 181-185.

Merckelbach, H., Dekkers, T., Wesset. I., \& Roefs, A. (2003). Dissociative Symptoms and Amnesia in Dutch Concentration Camp Survivors. Comprehensive Psychiatry, 44, 65-69.

Merckelbach, $H_{\text {.s }}$ Horselenberg, R., \& Muris, P. (2001). The Creative Experiences Questionnaire (CEQ): A brief self-report measure of fantasy proneness. Personality and Individual Differences, 31,987 995.

Merckelbach, H., Horselenberg, R., \& Sehmidt, H. (2002). Modeling the connection between self-reported trauma and dissociation in as student sample. Personality and Individnal Differences, 32, 695-705.

Merckelbach, H., \& Jelicic, M. (2004). Dissociative symptoms are related to endorsement of vague trauma ilems. Comprehensive Psychiatry, 45, 70-75.

Merckelbach, H., \& Muris, P. (2001). "The causal limk between self-teported trauma and dissociation: A critical review. Behawions Research and Therapy, 39, 245-254.

Merckelbach, H., Muris, P., Horselemberg. R., \& Stougie, S. (2000a). Dissociative experiences, response bías, and fantasy proneness in college students. Personality and Individual Differences. 28, 49-58.

Merckelbach, H., Muris, P., Nijman, H.\& \& de Jong, P. (1996). Self-reported cognitive failures and neurotic symptomatology. Personalify and individual Differences, 20,715-724.

Merckelbach, $\mathrm{H}$. Muris, P. \& Rassin, E. (1999). Fantasy proneness and cognitive failures as correlates of dissocitelive experienees. Personaliny and hidhidial Differences. 26,961-967.

Merckelbanch, H., Maris, P., Rassin, E. Horscienberg, R. (2000b). Dissociative experiences and interrogative suggestibility in collegie students. Personality and Individual Differences, 29,11331140.

Merckelbach, H., Rassin, E., \&uris, P. (2000c). Dissociation, schizotypy, and fantasy proneness in undergraduate students. Aourat of Nervous and Mental Disease, 188,428-431.

Merckelbach, H., Se Snith, G. P. (2003). Diagnostic accuracy of the Strtetured Inventory of Malingered Symptomatology (SMS) in detecting instructed malingering. Archives of Chricat Neuropsyehologry. $18,145-152$.

Mitchell, L. A., MacDonald, R. A., Brodie, E. E. (2004). Temperature and the cold pressor test. Journal of Poim. $5,233 \times 237$.

Modestin, J., E Emi, T. (2004). Testing the dissociative taxon. Psychiary Research, 126, 77-82.

Mulder, R. T. Beautrais, A. L., Joyce, P. R., \& Fergusson, D. M. (1998). Relationship between dissociation, childhood sexuad abuse, and mental iltness in a general population sample. American Jowrtat of Psychicary, 155, 806-811.

Muris, P., Mercketbach, H. (1997). Suppression and dissociation. Personality and Individual Differences. $23,523-525$.

Muris, P., Merckelbach, H., \& Peeters, E. (2003), The link between the Adolescent Dissociation Experiences Scale (A-DES), fantasy proneness, and anxiety symptoms. Journat of Nervous and Mental Disearie, 191, 18-24. 
Myake, A., Friedman, N. P., Emerson, M. I., Witzki, A. H., Howerter, A. (2000). The unity and diwersity of executive functions and their contribution to complex "frontall lobe tasks: A latent wariable analysis. Cognitive Psychology, 41, 49-100.

Nash, M. R., Hulsey, T. L., Sexton, M. C. Harralson, T. L. \& Lambert, W. (1993). Longutern sequelae of childhood sexual abuse: Percelved family envinonment, psychopathology, and dissociation. Jowr al of Consulting and Clincal Psychology, 61,276-283.

Neurosoft Inc. (2004). Neuroscan 4.3. Sterling, VA, USA.

Nijenhuis, E. R., Spinhoven, P., Van Dyck, R., Van der Hart 0 ., \& Vanderlinden, I. (1996). The development and psychometric characteristics of the Sonnatoforn Dissociation Questionnaire (SDQ-20). Joumal of Nervous and Mental Disease. 184, 688-694.

Nijenhuis, E. R., Spinhowen, P., van Dyck, R, wan der Hart, O., Vanderlinden, J. (1998). Psychonetric characteristics of the somatoform dissociation questionnaire: A replication study. Psychorherapy d Psychosomatics, 67, 17-23.

Nijenhuis, E. R., Spinhoven, P., Vanderlinden, J., van Dyck, R., \& wan der Hart, O. (1998). Somatoform dissociative symptoms as related to animal defensiwe reactions to predatory imminence and injury. Jownal of Abnomal Psychology 107, 63-73.

Nixon, R. D. V., Bryant, R. A., Moulds, M. L., Felmingham, K. L., \& Menstrodomenico, J. A. (2005). Physiologicall arousal and dissociation in acute trauma victims during trauma narratives. Journat of Traumatic Stress, 18, 107-113.

Ogawa, J. R., Sroufe, L. A., Weinfield, N. S., Carlson, E. A., \& Egeland, B. (1997). Dewelopment and the fragmented self: Longitudinal study of dissociative symptomatology in a nonclinical sample. Development and Psychopathology, 9, 855-879.

Pekala, R., Kumar, V. K., Ainslie, V. K., Eltiott, N. C. Mullen, K. J. Saninger. M. M., et al. (1999-2000). Dissociation as a function of child abuse and fantasy proneness in a substance abuse population. Imagination, Cognition and Personality, 19, 105i-129.

Pope, C. A., \& Kwapil, T. R. (2000). Dissociative experiences in hypothetically psychosis-prone college students. Joumal of Nervous and Mental Disease, 188, 530-536.

Pope, H. G., \& Hudson, J. I. (1995). Does childhood sexual abuse cause adult psychiatric disorders? Essential of methodology. Journal of Pswchiatry \& Law, 23, 363-381.

Pruessner, J. C., Kirschbaum, C., Meinlschmid, G., \& Hellhammer, D. H. (2003). Two formulas for: computation of the area under the curve represent measures of total homone concentration versus time-dependent change. Psychonewroendocrinology, 28, 916-931.

Putnam, F. W., Carlson, E. B., Ross, C. A., \& Anderson, G. (1996). Patterns of dissociation in clinicall and nonclinical samples. Journal of Nervots and Mental Disease, $184,673-679$.

Putnam, F. W., Helmers, K., Horowiiz, L. A., \& Trickett, P. K. (1995). Hypnotizability and dissociativity in sexually abused girls. Child Abuse and Neglect, 19, 645-655.

Rassin, E., Merckelbach, H., \& Spaan, V. (2001). When dreams become a royal road to confusion: Real istic dreams, dissociation, and fantasy proneness, Journal of Nervous and Mental Disease, 189, 478. 481 .

Reauschenberger, S. L.. L Lynn, S. J. (1995), Fantasy proneness, DSM-IIL R axis I psychopathology and dissociation. Journal of Abnormal Pisychology, 104, 373-380.

Ray, W., \& Faith, M. (1994). Dissociative experiences in a college age population. Personaldy and hadividual Differences, $18,223-230$.

Reinders, A. A., Nijenhuis, E. R., Paans, A. M., Korf, I., Willemsen. A. T. \& den Boer, J. A. (2003). One brain, two selves. Neuroimage, 20,2119-2125.

Rind, B., Tromowitch, $\mathbb{P}^{*}$., \& Bauserman, R. (1998). A meta-analytic examination of assumed properttes of child sexual abuse using college samples. Psychological Bulheth. 124, 22-53.

Robertson, I. H., Manly, T., Andrade, J., Baddeley, B. T., \& Yiend, J. (1997). "Oops': Performace correlates of eweryday attention failures in traumatic brain injured and normal subjects. Newopsychologia, 35, 747-758.

Ross, C. A., Ellason, J. W. \& Anderson, G. (1995). A factor analysis of the dissociative experieces scate (DES) in dissociative identity disorder. Dissociation, 8, 229-235.

Rossini, E. D., Schwartz, D. R., \& Braun, B. G. (1996). Intellectual functioning of inpatients with dissociative identity disorder and dissociative disorder not otherwise specified. Cognitive and neuropsychological aspects. Jowimal of Nervous and Menterl Disease. 184, $289-294$.

Russ, M. J., Campbell, S. S., Kakuma, T., Harrison, K., \& Zanine, E. (1999), EEG theta activily and pain insensitivity in self-injurious borderline patients. Psychiory Research, $89,201-214$.

Salkovskis, P. M. (1999). Understanding and treating obsessive-compulsive disorder. Behoviour Research and Therapy, $37, \$ 29-\$ 52$. 
Sandberg, D. A., 过ynn, S. J. (1992). Dissociatiwe experiences, psychopatholory and adjustment, and child and adolescent maitreatment in female college students. Jour pal of Abnornal Psychologw. 101, $717-723$

Sandberg, D. A. Lym, S. I. \& Matom, A. I. (2001). Information processing of an acquaintance rape scenario among high- and low-dissociating college women. Journal of Trownatic Sitress. 14, 585603.

Sanders, B. \& Giolas, M. H. (1991). Dissociation and childhood trauma in psychological disturbed adolescents. American Jownal of Psychatry; 148, 50-54.

Sanders, B., \& Green, A. (1994). The factor structure of dissociative experietices in college students. Distoctalion, $7,23-27$.

scaer, R. C. (2001). The neurophysiology of dissociation and oluronic disease. Applied Psychophysiology and Biofeculback, 26,79.91.

Schmid, R. G., Tirsch, W. S., \& Reitmeir, P. (1997), Correlation of developmental neurological findings with spectral analytical $\mathrm{EBG}$ evaluations in pre-school age children. Electroencephalography and Clinical Naterophysiology, 103, 516-527.

Semilitsch, H. V., Anderer, $P_{\text {., }}$ Sehuster, P., \& Presslich, O. (1986). A soletion for reliable and valid reduction of ocular artifacts, applied to the P300 ERP. Psychophysiology, 23, 695-703.

Sierra, M., \& Berrios, G. E. (1998). Depersonalization: Neurobiological perspectives. Biological Psychiatry. 44, 898-908.

Sierra, M., Senior, C, Dalton, J., McDonough, M., Bond, A., Phillips, M. L., el al. (2002). Autonomic response in depersonal ization disorder, Archives of General Psychiatry, 59, 833-838.

Silwa, C. E., \& Kirsch, 1. (1992). Interpretive sets, expectancy, fantasy proneness, and dissociation as predictors of hypnotie response. Joumal of Personality and Sociar Psychology, 63, 847-856.

Simeon, D. (2004). Depersonalisation disorder: A contempotary overview. CNS Drags, 18, 343-354.

Simeon, D., Guranik, O., Gross, S. Stein, D. J., Schmeidler, J., \& Hollander, E. (1998). The detection and measurement of depersonalization disorder. Journal of Nerwous and Memol Disease, 186, 536-542.

Simeon, D., Guralnik, O., \& Schmeidler, J. (2001). Development of a depersonalization severity scale. Joumal of Traumatic Strexs, 14,3411349

Simeon, D., Gurallnik, O., Schmeidler, J., Sirof, B., \& Knutelska, MA. (2001). The role of childhood interpersonal trauma in depersonalization disorder. American Journal of Psychiatry, 158, 10271033.

Simeon, D., Knutelska, M., Nelson, D. \& Guralnik, O. (2003). Feeling unreal A depersonalizatiom disorder update of 117 cases. Jownal af Chinical Psychiorry, 64, 990-997.

Simeon, D.. Knutelska, M., Nelson, D., Guralnik, O., \& Schmeidler, J. (2003). Examination of the pathological dissociation taxon in depersonalization disorder. Journal of Nenous and Mental Disease, $191,738-744$.

Smeets, T., Candel, I., \& Merckelbach, H. (2004). Accuracy, completeness, and consistency of emotional memories. American Joumal of Psychology, 117, 595-609.

Smith, G. P., \& Burger, G. K. (1997). Detection of malingering: Validation of the Structured Inventory of Malingered Symptomatology (SIMS). Jow wat of the Anerican Acadewy of Psychatry and the Law, 25, $183-189$.

Sobel, M. E. (1982). Astymptotic confidence intervals for indirect effect in structural equation models. In S. Leinhat (Ed.), Sociological models (pp. 290-3/2). San Francisco: Jossey-Bass.

Spiegel, D. \& Cardena, B. (1991). Disintegrated experience: The dissociative disorders revisited. Journal of Abrovmal Prychology 100, 366-378.

Spinhoven. P., wan der Does, A. (1999). Thought suppression, dissociation and psychopathology. Perwondity and lindividual Differencex, 27,877-886.

Sterlini, G. L., Bryant, R. A. (2002). Hyperarousal and dissociation: A stady of novice skydivers. Behowout Research and Therapy, 40,431-437.

Stickgold, R., Hobson, J. A., Fosse, R., \& Fosse, M. (2001). Sleep, learning, and dreams: Off-line memory reprocessing. Science, 294, 1052-1057.

Stroop. J. R. (193.5). Studies on interterence in serial verbal reactions. Joural af Experimental Psychology, $18,643-662$.

Surwillo, W. W. (1961). Frequency of the alpha whthm, reaction time and age. Nature, 19\%, 823-824.

Surwillo. W. W. (1963a). The relation of response-time variability to age and the influence of brain wave frequency. Electroencephalography and Clinical Neurophysiology, 15, 1029-1032.

Surwillo, W. W. (1963b). The relation of simple response time to brain-wave frequency and the effects of age. Electroencephalography and Chincal Neurophysiology, 15, 105-114.

Tampke, A. K., \& Irwin, H. J. (1999). Dissociative processes and symptoms of postraumatic stress in Vietnam Velerans. Aournal of Traumatic Siress. 12,725-738. 
Tanaka, H., Hayashi, M.. \& Hori, T. (1997). Topographical characteristics and principal componem strueture of the hypnagogic EEG. Sleep, 20,523 -534.

Tomarken, A. J., Dawidson, R. J., Wheeler, R. E. \& Kinney, L. (1992). Psychonetric properties of resting anterior EEG asymmetry: Temporal stability and internal consistency. Psyohophysiology, 29,576592.

Tumer, M. L., \& Engle, R. W. (1989). Is working memory capacity task dependent? Journd of Memory and Language, $28,127-154$.

van den Hout, M., Merckelbach, H., \& Pool, K. (1996). Dissociation, realify monitoring, trauma, and thought suppression. Behaviowral and Cognitive Psychotherapy, 24, 97.108.

wan der Kolk, B. A., \& Fisler, R. (1995). Dissociation and the fragmentary nature of traumatio memories: Overview and exploratory study. Noumal of Tramonic Stress, $8,505-525$.

van der Kolk, B. A. \& van der Hart, O. (1989). Pierre Janet and the breakdown of adaptation in psychological trauma. American Journal of Psychiatry, 146, 1530-1540.

van IJzendoorn, M. H., \& Schuengel, C. (1996). The measurement of dissociation in nomal and clinical populations: Meta-analytic validation of the Dissociative Experience Scale (DES). Clinical Psychology Review, 16, 365-382.

Vanderlinden, J., Vandereycken, W., wan Dyck, R., \& Vertommen, H. (1993). Dissociative experiences and trauma in eating disorders. International Journal of Eating Disorders. $83,187-193$.

Verschuere, B., Crombez, G., Clercq, A., de, \& Ernst, H. W. (2004). Autonomic and behavional tesponding to concealed information: Differentiating orienting and defensive responses. Psychophysiology. $461-466$

Vogt, F., Klimesch, W., \& Doppelmayr, M. (1998). High-frequency components in the alpha band and memory performance. Journal of Clinical Neurophysiology 15, 167-172.

Wald, F. D. M., Mellenberg. G. J. (1990). De verkorte versie wan de Nederlandse vertaling van de Profile of Mood States (POMS). [The brief version of the Duteh translation of the Profile of Mood States (POMS)]. Nederlands Tijdschiff Voar de Psychologie [Dutch Jow af af Pychology. 45, 86-90,

Waldio, T. G., \& Merritt, R. D. (2000). Family proneness, dissociaton, and DSM-IV Axis II symptomatology. Journal of Abnormal Psychology, 109, 555-558.

Waller, G.. Ohanian, V., Meyer, C., Everill, J., \& Rouse, H. (2001). The utility of dimensional and categorical approaches to understanding dissociation in the eating disorders. British Journal of Climical Psychology, 40, 387-397. Psychology, 40, 387-397.
Waller, N. G. (1995). The Dissociative Experiences Scale. In J. C. Conoley \& I. J.C. (Eds.), Twelyh mental
measurewents yearbook. Lincoln: University of Nebraska Press.

Waller, N. G., Putnam, F. W., \& Carlson, E. B. (1996). Types of dissociation and dissociative types: A taxometric analysis of dissociative experiences. Psychological Methods. 1, 300-321.

Waller, N. G., \& Ross, C. A. (1997). The prevalence and biometric structure of pathological dissociation in the general population: Taxometric and behavior genetic findings. Jourmal of Abwormal Psychology, 106, 499-510.

Watson, D. (2001). Dissaciation of the night: Individual differences in sleep-related experiences and their relation to dissociation and schizotypy. Journal of Abnomal Psychology, 110,526-535.

Watson, D. (2003a). Investigating the construct validity of the dissociative taxon: Stability analy is of normal and pathological dissociation. Journal of Abnormal Psychology, 112, 298-305.

Watson, D. (2003b). To dreant, perchance to remember: Indiwidual differences in dream recall. Persoritatity and Individual Differences, 34, 1271-1286.

Wegner, D. M., Schneider, D. I., Carter, S. R., \& White, T. L. (1987). Paradoxical elfects of thought suppression. Uournal of Personalioy and Social Psychology, 53,5-13.

Wegner, D. M., \& Zanakios, S. (1994). Chronic thought suppression. Jow Fat of Personality, 62, 615 -640.

Williams, C. L., Haines, J., \& Sale, I. M. (2003). Psychophysiological and psychological correlates of dissociation in a case of dissociative identity disorder. Joumal of Tramma and Dissocianom. 4 , $101-118$ 101-118.
Williams, J. M. G. Mathews, A., \& MacLeod, C. 1996), The emotional Siroop task and psychopathology.
Psychological Bullerin. 120, 3-24.

Williams, M. A., Moss, S. A., Bradshaw, J. L., \& Rinehart, N. J. (2002). Random number generation in autism. Journal of Autism and Developmental Disorders, 32,43-47.

Williamson, A. M., Feyer, A. M., Mattick, R. P., Friswell, R., \& Finlay Brown, S. (2001). Developing measures of fatigue using an alcohol compatison to validate the effects of fatigue on performance Accident Analysis and Prevenition, 33, 313-326.

Winkielman, P., Schwarz, N., \& Belli, R. F. (1998). The role of ease of retrieval and atribution in menory judgments: Judging your memory as worse despite recalling more evenis. Psychological Science. 9, $124-126$. 
Wolfe, J., Chrestman, K. R., Crosby Ouimete, P., Kalloupek, D., Harley, R. M. 2 Bucsela, M. (2000). Traumerelated psychophystological reactivity in women exposed to war-zone stress. Joumal of Clibical Prychologis, 56, 1371-1379.

Wolfad, U. \& Meyer, T. (1998). Interrogative suggestibility, anxiety and dissociation anong anxious patients and normal controls. Persondity and Indiwidual Differences, $25,425-432$.

Wright, D. B. Lofus, E. W. (1999). Measuring Dissociation: Comparison of alternative forms of the Dissociative Experiences Scall. Anerican fournal of Psychology. $112,497-519$.

Zlotnick, $\mathbb{C}_{*}$, Shea, M. T., Pearlstein, T., Simpson, E. Costello, E., \& Begin, A. (1996). The relationship between dissociative symptoms, alexithymia; impulsivity, sexual abuse, and sellf-mutilation. Comprehensive Psichiatry, 37, 12-16.

Zoroglu, S. S., Tuzun, U., Sar, V., Tutkun, H, Savas, H. A., Ozturk, M., et al. (2003). Suicide attempt and self-muttlation among Turkish high school students in relation with abuse, neglect and dissociation. Psychatry and Chical Newrosctences. 57, 119-126. 


\section{Summary}

One assumption, commonly held in the clinical literature, is that dissociative experiences serve a purpose in that these experiences are the manifestation of a defense mechanism. This postulation, however, rests on a weak empirical basis (Kihlstrom, 2005). The present dissertation critically evaluates the "dissociation-as-a-defensemechanism" notion.

Chapter 1 reviews the existing literature on the relation between dissociation and trauma. It demonstrates why the heavy reliance on self-reports of traunatic experiences in the literature, which appears to support the notion that dissociation is caused by exposure to traumatic events, is problematic. Studies relying on objective measures of dissociation did, in fact, fail to support these findings. In addition, we highlight why the overlap between dissociation and fantasy proneness and cognitive failures might confound findings relying on retrospective self-reports. Moreover, specific predictions deduced from the notion that dissociation is the manifestation of a defense mechanism are postulated. These predictions will be investigated in subsequent chapters. Last but not least, we present an alternative view for the development of dissociative experiences. This view states that disruptions in the sleep-wake cycle might give rise to dissaciative experiences.

If dissociation is the manifestation of a defense mechanism, one would expect individuals with heightened levels of dissociation to exhibit decreased physiological responsivity, as compared to low dissociatiors. Part I scrutinizes this prediction, while employing two different measures of physiological responsivity.

Chapter 2 describes a study that measured the influence of trait dissociation on the skin conductance response to an aversive video fragment. Contrary to the supposedly defensive function of dissociation, heightened levels of dissociation were accompanied by heightened skin conductance responses. In addition, memory for the video fragment was tested. In line with previous research (Candel et al., 2003), dissociation was not associated with amnesia (i.e., less hits), but with a heightened tendency to confabulate.

Chapter 3 reports on two studies that induced acute stress by means of a Trier Social Stress Test (TSST; Kirschbaum et al., 1993). In both studies, trait dissociation was unrelated to a suppression of physiological responsivity, as indexed by the cortisol response. In line with results from chapter 2 , high dissociators experienced more subjective stress.

Part // critically examines the assumption that dissociation is a selective attentional deviation to aversive stimuli.

Chapter 4 presents the findings of a study employing a modified version of the Stroop color naming task containing emotional as well as neutral stimuli. Unexpectedly, individuals that are high on dissociation reacted slower to all stimulus word, irrespective 
of their valence. Therefore, dissociation could probably best be regarded as a global deficit rather than a selective attentional deviation to aversive stimuli.

Chopter 5 investigated whether dissociation is related to subtle deficits in executive functioning. To this end, the Random Number Generation Task, which asks the participant to generate random sequences of numbers, was employed. The idea that dissociation might be related to minor disruptions in executive functioning was supported.

Chapter 6 describes a study that tested the hypothesis that individuals who are high on dissociation should be better at suppressing aversive information as compared to low dissociators. However, the opposite appears to be true, as a trend emerged with individuals high on dissociation experiencing more intrusion of an emotional video fragment than low dissociators.

In part $I I /$ an alternative idea for the emergence of dissociative experiences is investigated, assuming that dissociation might be fuelled by disruptions in the sleep-wake cycle.

Chaprer 7 presents a study that tests the aforementioned relation between sleep experiences and dissociation. While dissociation was related to various sleep experiences such as nightmares, it was neither related to lucid dreaming nor to a particular sleeping pattern (morningness vs. eveningness).

Chapter 8 investigated the possibility that the robust relation between dissociation and fantasy proneness might stem from disruptions in the sleep-wake cycle. This idea is underpinned by a mediational analysis, which shows that the relation between dissociation and fantasy proneness is at least partially due to sleep experiences.

Chapter 9 sought to investigate the relation between dissociation and sleep, while quantifying disruptions of the sleep-wake cycle independent of self-report. Resting EEG was measured in addition to subjective sleep experiences. While we did find the expected relation between dissociation and self-reported sleep experiences, these selfreported sleep-experiences were not related to the power in the resting EEG.

Dissociation, however, appears to be related to an increase in the $\theta$-power range and a suppression in the $\alpha$-power range. Interestingly, some authors related these deviations both to inferior performance on menory and attention tasks (Klimesch, 1999), as well as the hypnagogic stage (Tanaka et al., 1997).

In the general discussion the main findings from this dissertation are summarized and future research avenues are presented. 


\section{Samenvatting}

In de klinische literatuur heerst de mening dat dissociatieve ervaringen de manifestatie van een doelbewust defensie mechanisme zijn. Deze assumptie mist echter een stevige empirische verankering (Kihlstrom, 2005). Daarom onderwerpen wij deze opvatting aan een kritische blik.

Hoofdstuk I geeft een overzicht van de empirische literatuur die de relatie tussen dissociatie en trauma onderzoekt. Tevens wordt er uitgelegd warom het problematisch is dat veel van deze studies, berusten op retrospectieve trauma rapportages. Studies die daarentegen gebruik maakten van objectieve maten van trauma slaagden er namelijk niet in om deze samenhang te vinden. Deze discrepantie in bevindingen zou het gevolg kunnen zijn van de overlap tussen dissociatie, fantasierijkheid en cognitieve fouten. De neiging tot cognitieve fouten en fantasierijkheid zouden samen als confounder kunnen fungeren en tot een kunstmatige inflatie van de bevindingen geleid kunnen hebben. Bovendien leiden wij een aantal voorspellingen af uit de notie dat dissociatie inderdaad de manifestatie van een defensie mechanisme zou zijn. Deze zullen worden getoetst in de volgende hoofdstukken.

Als dissociatie de manifestatie van een defensie mechanisme is, zou men verwachten dat individuen die hoog scoren op dissociatie minder fysiologische reacties zullen vertonen dan laag dissociatieve personen. In deel $l$ onderzochten we deze voorspelling door middel van twee fysiologische maten.

Hoofdstuk 2 beschrijft een studie die de invloed van de karaktertrek dissociatie op de huidgeleidingsrespons tijdens een aversief video fragment meet. In tegenstelling tot de veronderstelde defensieve functie van dissociatie waren hoge niveaus van dissociatie gerelateerd aan een hogere huidgeleidingrespons. Ook onderzochten wij het geheugen voor het videofragment. In overeenstemming met eerder onderzoek (Candel et al., 2003) vonden we dat dissociatie niet gekenmerkt was door amnesie (minder hits), maar door een verhoogde tendens om te confabuleren.

Hoofdstuk 3 rapporteert twee studies die acute stress induceerden door middel van een Trier Social Stress Test (TSST; Kirschbaun et al., 1993). Tijdens beide studies ging dissociatie niet samen met een suppressie van de fysiologische arousal, gemeten door middel van de cortisol respons. Echter, in overeenstemming met hoofdstuk 2, vertoonden hoog dissociatieve personen meer subjectieve stress.

Deel II beantwoordt de vraag of de assumptie dat dissociatie zou moeten worden beschouwd als een selectieve deviatie van de aandacht als respons op aversieve stimuli, gerechtvaardigd is.

Hoofdstuk 4 rapporteert de bevindingen van een onderzoek dat gebruik maakt van een gemodificeerde versie van de Stroop taak die uit zowel aversieve als ook neutrale stimuli bestond. Dissociatie leek tijdens deze taak geen sellectieve, d.w.Z. alleen tijdens aversieve stimuli, invloed uit te oefenen, maar ging samen met een algehele vertraging tijdens alle stimuli, ongeacht hun valentie. Daarom zou dissociatie mogelijk beter 
kunnen worden omschreven als een globaal gebrek in plaats van een selectieve deviatie als respons op aversieve stimuli.

Hoofdstuk 5 onderzocht of dissociatie gerelateerd is aan subtiele deficiënties in executieve functies. Hiervoor maakten wij gebruik wan de Random Number Generation Task, die aan de deelnemers vraagt om toevallige reeksen van getallen te genereren. Onze bevindingen onderstrepen het idee dat dissociatie samen gaat met een globaal gebrek, in dit geval lichte verstoringen van executieve functies.

Hoofdstuk 6 onderzoekt de voorspelling dat individuen die meer dissociatieve ervaringen rapporteren, minder last zouden hebben van intrusies van aversieve informatie waaraan ze eercler bloot gesteld werden. Echter, het tegenovergestelde bleek waar te zijn. Individuen die veel dissociëren bleken namelijk juist meer intrusies van het emotionele videofragment te rapporteren dan individuen die weinig dissociëren.

Deel III volgt een ander pad, want hier wordt een alternatieve opvatting over de herkomst van dissociatieve ervaringen onderzocht. Dit is het idee dat dissociatieve ervaringen voortkomen uit een verstoord slaap-waak ritme.

Hoofdstuk 7 presenteert een studie die de eerder genoemde relatie tussen slaapervaringen en dissociatie toetst. Onze bevindingen laten zien dat dissociatie samen gaat met verscheidene algemene slaapervaringen zoals nachtmerries, maar noch gerelateerd is aan een bepaald slaappatroon (ochtend of avond persoon), noch aan lucide dromen.

Hoofdstuk 8 onderzoekt de mogelijkheid dat de overlap tussen dissociatie en fantasierijkheid gemedieerd wordt door verstoringen in het slaap-waak ritme. Onze mediatie analyse laat dan ook zien dat deze slaapervaringen een deel van de relatie tussen dissociatie en fantasierijkheid kunnen verklaren.

Hoofdstuk 9 poogt de bevindingen uit hoofdstuk 7 en 8 te ondersteunen door verstoringen in het slaap-waak ritme objectief in kaart te brengen. Daarom maten wij naast subjectieve slaapervaringen ook nog rust EEG. Net als in hoofdstuk 7 en 8 vonden wij dat dissociatie samen ging met subjectieve slaapervaringen. Deze subjectieve slapervaringen waren niet gerelateerd aan de power in het rust EEG. Dissociatie bleek echter wel samen te gaan met een verhoogde power in de $\theta$-band en een suppressie in de $\alpha$-band. Dit is interessant, omdat eerder onderzoek deze deviatie relateert aan inferieure prestatie op geheugen- en aandachtstaken (Klimesch, 1999), maar ook aan de hypnagoge fase (Tanaka et al., 1997).

De algemene discussie zet de hoofdbevindingen nog eens op een rijtje en toont mogelijkheden voor toekomstig onderzoek op het terrein van dissociatie. 


\section{Zusammenfassung}

In der klinischen Literatur wird regelmäßig unterstellt, dass dissoziative Erfahrungen die Manifestation eines defensiven Mechanismus sind. Diese Annahme basiert jedoch auf einer fragwürdigen empirischen Basis (Kihlstrom, 2005). Die Annahme, dass dissoziative Erfahrungen defensive Eigenschaften beinhalten, wird in dieser Dissertation hinterfragt und empirisch untersucht.

Kapitel 1 liefert einen kritischen Überblick über den Zusammenhang zwischen Trauma und Dissoziation. Studien, welche diesen Zusammenhang untersuchen, werden präsentiert und Schwachpunkte hierbei aufgezeigt. Ferner werden verschiedene Persönlichkeitsmerkmale, die im Zusammenhang mit Dissoziation stehen, untersucht und die Möglichkeit diskutiert, dass diese zum Auftreten von Scheinkorrelationen zwischen einem retrospektiv angegebenem Trauma und Dissoziationen fuhren (könnten).

Wir kommen hierbei zum Ergebnis, dass der konstatierte Zusammenhang viel weniger offensichtlich und eindeutig ist, als dies bisher in der Literatur dargestellt wird. Auch leiten wir mehrere, der hieraus abgeleiteten Prognosen von der Schlussfolgerung ab, dass dissoziative Erfahrungen die Manifestation eines Verteidigungsmechanismus seien. Diese Prognosen werden in den folgenden Kapiteln empirisch überprüft. Den Abschluss des Kapitels bildet eine alternative Auffassung, welche dass Entstehen von Dissoziation in Zusammenhang mit Störungen des Schlaf-Wach-Zyklus bringt.

Wenn Dissoziation die Manifestation eins Abwehrmechanismus ist, dann wäre zu erwarten, dass Personen mit erhöhten Frequenzen von dissoziativen Erfahrungen weniger stark ausgeprägte physiologische Reaktivitäten zeigen, wenn sie mit enotionell negativen Situationen konfrontiert werden. Die dieser These entsprechende Prognose wird in Teil $l$ dieser Dissertation mit Hilfe verschiedener physiologischen Parameter untersucht.

Kapitel 2 beschreibt eine Studie, die den Einfluss des Charakterzuges Dissoziation auf die Hautwiderstandsreaktion während eines emotionellen Videofragments untersucht. Im Gegensatz zur defensiven Funktion von Dissoziation, die oft in der klinischen Literatur beschrieben wird, zeigten Individuen mit einem hohen Dissoziationsniveau eine stärkere Hautwiderstandsreaktion als solche mit einem geringeren Dissoziationsniveat.

Kapitel 3 berichtet über zwei Studien, in weichen mit Hilfe eines Trier Social Stress Tests akuter Stress induziert wurde. In beiden Studien waren Dissoziationsniveaus unabhängig von einer Unterdrückung der physiologischen Reaktivität, die wir mit Hilfe der Cortisolreaktion maßen. Jedoch berichteten Teilnehmer mit einem hohen Dissoziationsniveau hierbei subjektiv über größere Stresserfahrtungen.

Teil II dieser Dissertation beschäftigt sich mit der Frage, ob die Annahme gerechtrertigt ist, dass Dissoziation mit einer selektiven Abweichung auf dem Gebiet der Aufmerksamkeit zusammenhängt. 
Kapifel 4 präsentiert die Befunde einer Studie, die von einer modifizierten Stroop Aufgabe mit emotionell negativen und neutralen Stimulus-Wörtern Gebrauch macht. Im Gegensatz zu den Prognosen, die regelmäßig in der klinischen Literatur gefunden werden, reagierten Teilnehmer mit hohen Dissoziationsniveaus langsamer auf sämtliche Stimuli, unabhängig von deren Valenz. Ausgehend von diesem Befund muss Dissoziation folglich als eine globale Abweichung auf dem Gebiet der Aufmerksamkeit angesehen werden.

Kapitel 5 untersucht, ob Dissoziation mit subtilen Abweichungen auf dem Gebiet des exekutiven Funktionierens zusammenhängt. Hierfür stützen wir uns auf die Random Number Generation Task. In der entsprechenden Aufgabe werden die Teilnehmer gebeten, zufallige Zahlensequenzen zu generieren. Die Befunde dieser Studie stützen die Vermutung, dass dissoziative Erfahrungen mit Abweichungen auf dem Gebiet des exekutiven Funktionierens zusammenhängen.

Kapitel 6 beschreibt eine Studie, wellche die Hypothese prüfte, dass Individuen die hoch dissoziativ sind besser die Gedanken an negative Informationen unterdrücken können, welche wäharend der Untersuchung in der Form eines emotionellen Videos präsentiert wurden. Unsere Daten legen jedoch nahe, dass Individuen mit hohen Dissoziationsniveaus erstaunlicherweise diese Gedanken schlechter unterdrücken können als lndividuen mit niedrigen Dissoziationsniveaus.

In Teil III wird eine alternative Hypothese zur Entstehung von Dissoziation gepruft . Nämlich die Möglichkeit, dass Störungen des Schlaf-Wach-Zyklus die Ursache dieser Erfahrungen sind.

Kapitel 7 präsentiert eine Studie zur Beziehung zwischen Dissoziation und verschiedenen Schlaferfahrungen. Hierbei zeigt sich ein Zusammenhang zwischen Dissoziation und unterschiedlichen Schlaferfahrungen, z.B. Albträumen, nicht aber mit luziden Träumen oder einem bestimmten Schlafmuster (Morgenmensch oder Abendmensch).

Kapitel 8 untersucht die Möglichkeit, dass der robuste Zusammenhang zwischen Fantasieneigung und Dissoziation durch Störungen des Schlaf-Wach-Zyklus verursacht sein konnte. Eine Mediationsanalyse unterstüzt diese Annahme da ein Teil des Zusammenhangs zwischen Fantasieneigung und Dissoziation auf diese Schlaferfahrungen zurilickzuführen ist.

Kapitel 9 beschreibt eine Studie, die darauf abzielt, den Zusammenhang zwischen Dissoziation und Schllaf unabhängig von selbst angegebenen Schlaferfahrungen zu quantifizieren. Darum zeichneten wir außer den subjektiven Schlaferfahrungen auch noch ein Ruhe-EEG auf. Wie erwartet zeigt sich ein Zusammenhang zwischen subjektiven Schlaferfahrungen mit Dissoziation, nicht aber mit den EEG-Ableitungen. Jedoch besteht ein Zusammenhang zwischen Dissoziation und einer Zunahme der Aktivität im Alpha-Band und einer Abnahme im Theta- und Delta-Band. Diese Abweichungen werden von einigen Autoren mit geringer Leistung auf dem Gebiet der Aufmerksamkeit und des Gedächtnisses (Klimesch, 1999), sowie mit der hypnagogen Phase (Tanaka, Hayshi \& Hori, 1997) in Zusammenhang gebracht. 
In der Allgemeinen Diskussion werden schließlich die wichtigsten Befunde dieser Dissertation zusammengefasst. Den Abschluss unserer Überlegungen bilden Forschungsdesiderate. 


\section{Dankwoord}

Een proefschrift schrijf je niet alleen en zeker niet zonder hulp. Daarom zou ik heel wat mensen willen bedanken voor hun steun, hun ideeën, hun kritiek of simpelweg de gezelligheid.

Harald wil ik härtelijk bedanken voor de gesprekken die wij samen hadden en alle inspiratie die daaruit kon putten voor mijn onderzoek, maar ook woor het in recordtempo corrigeren van mijn stukken met de meedogenloze rode pen.

De andere collega's van PsyLaw: Kim, Elke, Saskia, Ewout, Beatrijs, Maarten, Tom, Ingrid, Robert en Marco. Het heeft mij heel erg geholpen dat ik jullie altijd lastig mocht vallen met ingewikkelde maar ook banale vragen. Bovendien heb ik altijd heel van de frequente kopjes koffie en de roddels met jullie genoten.

Het IRP aan de overkant: Hans, Peter, Han en Wilma. Bedankt daarvoor dat jullie altijd. vroegen: "En wat heeft de rechter er nu aan?" en mij er dus bij tijden aan herinnerden om er ook naar te kijken wat je met je bevindingen zou kunnen betekenen.

Alle collega"s van Experimentele Psychologie voor de gezellige, maar toch inspirerende sfeer met name in de buurt van het koffiezetapparaat.

Alle studenten die tijdens hun stage, jaarwerkstukken, andere onderzoeken of als student-assistenten bij mij meegedacht en meegeholpen hebben: $2 \times$ Elke, $2 \times$ Ellen, Gwenny, Sven, Nikki, Karin, Marieke, Sander, Sophie, Clint, Mi Mi, Frauke, Nora, Michelle, Anne, Maartje, Linda, Maud, Katja, Alica, Ilse, Hannah, Anouk, Myrte, Olga en Charlotte.

Ewout en Ulf: Leuk dat jullie mijn paranimfen willen zijn.

Natürlich darf und möchte ich auch meine Eltern und meine Omas und Opas nicht vergessen. Heidy, Arno, Lilli, Maria, Waldemar und Ludwig, vielen Dank für alle Unterstïtzung und die vielen Dinge die ich von Euch gelernt habe.

Tia, bedankt voor alles! 


\section{Curriculum Vitae}

Timo Giesbrecht was born on 7. Juli 1977 in Aachen, Germany. In July 1997, he graduated from secondary school, Anne Frank Gymnasium Laurensberg, Aaclen, Germany. Thereafter, he fulfilled his community service at the daycare section of St. Raphael home for elderly people. In 1998, he began studying psychology at the Maastricht University, where he received his master degree in cognitive psychology in 2002. He conducted the research for his master thesis at Humboldt University of Berlin, Germany. In 2002, he began his $\mathrm{PhD}$ project on the defensive function of dissociative experiences at the Maastricht University. His PhD project was supported by a grant from the Dutch organisation for scientific research N.W.O., grant number 402-01-088-D. 


\section{Publications}

\section{Journal articles}

Giesbrecht, T. \& Merckelbach, H. (in press). Dreaming to reduce fantasy - Fantasy proneness, dissociation and subjective sleep experiences. Personality and Individual Differences.

Giesbrecht, T., Jongen, E., Smulders, F., \& Merckelbach, H. (in press). Dissociation, cortical activity, and sleep. Journal of Nervous and Mental Disease.

Merckelbach, H., Zeles, G., van Bergen, S., \& Giesbrecht, T. (in press). Trait dissociation and commission errors in memory reports of emotional events. American Journal of Psychology.

Giesbrecht, T. \& Merckelbach, H. (2006). Dissociatieve Symptomen en Slaap. Tijdschrift voor Psychiatrie, 48, 207-21.5.

Merckelbach, H. \& Giesbrecht, T. (2006). Subclinical dissociation, schizotypy, and traumatic distress. Personality and Individual Differences, 40, 365-374.

Giesbrecht, T. \& Merckelbach, H. (2005). Über die kausale Beziehung zwischen Dissoziationen und Trauma: Ein kritischer Uberblick. Der Nervenarzt, 20-27.

Merckelbach, H., à Campo, J., Hardy, S., \& Giesbrecht, T. (2005). Dissociation and fantasy proneness in psychiatric patients: A preliminary study. Comprehensive Psychiatry, 46, 181-185.

Giesbrecht, T., Merckelbach, H., Geraerts, E., \& Smeets, E. (2004). Disruptions in executive functioning and dissociation in undergraduate students. Journal of Nervous and Mental Disease, 192, 567-569.

Giesbrecht T. \& Merckelbach, H. (2004). Subjective sleep experiences are related to dissociation. Personality and Individual Differences, 37, 1341-1345.

Merckelbach, H., Jelicic, M., Giesbrecht, T., \& Horselenberg R. (2003). De wraak van Hughlings Jackson. Over gesimuleerde 'fugue'. Maandblad Geestelijke volksgezondheid, 58, 654-665.

\section{Submitted manuscripts}

Giesbrecht, T., Geraerts, E., \& Merckelbach, H. (submitted for publication).

Dissociation, memory commission errors, and heightened autonomic reactivity.

Giesbrecht, T., Smeets, T., Merckelbach, H., \& Jelicic, M. (submitted for publication).

Trait dissociation and stress induced cortisol responses.

Giesbreclit, T. \& Merckelbach, H. (subnitted for publication). Critically evaluating the betrayal trauma theory of dissociative experiences - A negative finding.

Giesbrecht, T., Merckelbach, H., \& Smeets, E. (submitted for publication). First you find them, then you don't - Thought suppression, dissociation, and context effects.

Giesbrecht, T., Smeets, T., \& Merckelbach, H. (submitted for publication).

Dissociative experiences on ice - Peritraumatic and trait dissociation during the cold pressor test. 


\section{Conference presentation}

Giesbrecht, T. \& Merckelbach, H. (2003). Dissociative experiences in undergrciduate students reflect lack of cognitive efficiency. Poster presented at the Conference of the Society for Applied Research in Memory and Cognition, Aberdeen, Great Britain.

Giesbrecht, T., Geraerts, E., \& Merckelbach, H. (2004). Dissociation, memory" commission errors, and heightened autonomic reactivity. Paper presented at the European Conference on Psychology and Law, Krakow, Poland.

Giesbrecht, T., Geraerts, E., \& Merckelbach, H. (2004). Dissociation, memory commission errors, and heightened autonomic reactivity. Poster presented at the Tagung der Experimentell arbeitenden Psychologen, Giessen, Germany.

Giesbrecht, T., Jongen, E., Smulders, F., \& Merckelbach, H. (2005). Dissociation, cortical activity, and sleep. Paper presented at the Conference of the Society for Applied Research in Memory and Cognition, Wellington, New Zealand.

Giesbrecht, T., Jongen, E., Smulders, F., \& Merckelbach, H. (2005). Dissociation. cortical activity, and sleep. Poster presented at the Tagung der Experimentell arbeitenden Psychologen, Regensburg, Germany. 